JOURNAL OF THE

AMERICAN MATHEMATICAL SOCIETY

Volume 12, Number 1, January 1999, Pages 173-240

S $0894-0347(99) 00279-9$

\title{
PERIODS OF AUTOMORPHIC FORMS
}

\author{
HERVÉ JACQUET, EREZ LAPID, AND JONATHAN ROGAWSKI
}

\section{Contents}

I. Introduction 174

II. Integrals over cones 178

III. Automorphic forms and truncation 185

1. Roots, coroots, etc. 185

2. Inversion formulas 186

3. The standard characteristic functions 187

4. Automorphic forms 188

5. Truncation operators 189

IV. Periods relative to quadratic extensions 189

6. Mixed truncation 190

7. The period of an automorphic form 191

8. Period of a truncated automorphic form 198

9. Eisenstein series 202

V. Integral of an automorphic form 204

10. Integral of a truncated automorphic form 205

11. Integral of a truncated Eisenstein series 206

12. General inner product formula 207

VI. Regularized periods of a cuspidal Eisenstein series on $G L(n) \quad 209$

13. Weyl groups and double cosets 209

14. Invariant functionals on principal series representations 212

15. The regularized period 212

VII. Intertwining periods 221

16. Minimal involutions 222

17. Graph of involutions 222

18. The functional equations 224

19. A description of $\Omega\left(\xi, \xi^{\prime}\right) \quad 229$

20. Unramified computations $\quad 229$

VIII. Periods of truncated Eisenstein series 236

$\begin{array}{ll}\text { References } & 238\end{array}$

Received by the editors October 14, 1997 and, in revised form, May 14, 1998.

1991 Mathematics Subject Classification. Primary 11F55, 11F70, 11F72.

The first author was partially supported by NSF Grant 9619766.

The third author was partially supported by NSF Grant 9401466.

(C)1999 American Mathematical Society 


\section{INTRODUCTION}

Let $G$ be a reductive group over a number field $F$, and let $H \subset G$ be a subgroup obtained as the fixed point set of an involution $\theta$. Then the following period integral

$$
\Pi^{H}(\varphi)=\int_{H(F) \backslash H(\mathbb{A})^{1}} \varphi(h) d h
$$

converges absolutely for any cusp form $\varphi$ on $G(\mathbb{A})$. Our first goal in this paper is to develop a method for defining and computing $\Pi^{H}(\varphi)$ where $\varphi$ is a more general automorphic form such as an Eisenstein series. In this case, the integral need not converge and we define it by means of a regularization procedure. Our second goal is to use this regularized period to obtain explicit formulas for the (convergent) period $\Pi^{H}\left(\Lambda^{T} E\right)$ where $\Lambda^{T} E$ is a truncated Eisenstein series.

Before describing the contents of this article, let us recall some motivation. The periods $\Pi^{H}\left(\Lambda^{T} E\right)$ are of interest because they appear in the relative trace formula (RTF) in a role analogous to that played by inner products of truncated Eisenstein series in the Arthur-Selberg trace formula. They arise when one computes the contribution of the continuous spectrum to the relative trace formula (cf. [J2], [J3] for an overview of the RTF). The relative trace formula itself has been used in a variety of applications, beginning with the work of Jacquet-Lai on periods of Hilbert modular forms ([JL], [LA1], also [FH]) and Jacquet on Waldspurger's theorems [J1] (cf. also [GU]). It provides a general tool for studying distinguished representations. Recall that a cuspidal representation $\left(\pi, V_{\pi}\right)$ of $G$ is said to be distinguished by $H$ if $\Pi^{H}(\varphi) \neq 0$ for some $\varphi \in V_{\pi}$. In some cases, periods of this type have an interesting topological or arithmetic interpretation (cf. [HLR], [BR]). Actually, in applications, the notion of period integral must be suitably refined: in general, it may have the form:

$$
\int_{Z H(F) \backslash H(\mathbb{A})} \varphi(h) \chi(h) d h
$$

where $Z$ is a central subgroup of the ambient group and $\chi$ is a character of $H(\mathbb{A})$ trivial on $H(F)$. In many cases, it should be possible to characterize the $H$ distinguished cuspidal representations as images with respect to a functorial transfer to $G$ from a third group $G^{\prime}$. General results of this type, which are known in many cases, should eventually follow from a comparison of suitable relative trace formulas on $G$ and $G^{\prime}$. The RTF thus provides a link between certain cases of the functoriality conjecture and period integrals.

A candidate for $G^{\prime}$ over the algebraic closure of $F$ was proposed in [JLR]. In [G] this has been refined to a description of $G^{\prime}$ as an $F$-group. Suppose, for example, that $G=\operatorname{Res}_{E / F} H$ where $E / F$ is a quadratic extension and $\theta$ is the involution induced by Galois conjugation relative to $E / F$. This is the case we focus on in this article (although our methods generalize; we intend to deal with the general case in a future article). If $H$ is also simple and split, then $G^{\prime}$ will be either $H$ itself or the unique quasi-split outer form of $H$ that splits over $E$, according as -1 lies in the Weyl group of $H$ or not ([G]). Thus if $H=G L(n)_{/ F}$, then $G^{\prime}$ is the quasi-split unitary group $U(n)$ (see also [F1] and [F2]) and if $H=S p(n)_{/ F}$, then $G^{\prime}=S p(n)_{/ F}$. The relative trace formula in this situation is based on the equality 
of geometric and spectral expansions of the integral

$$
\int_{H(F) \backslash H(\mathbb{A})^{1}} \int_{N(E) \backslash N\left(\mathbb{A}_{E}\right)} K_{f}(h, n) \psi(n) d h d n
$$

where $K_{f}(x, y)$ is the automorphic kernel attached to a function $f$ on $G(\mathbb{A}), N$ is the unipotent radical of the standard Borel subgroup, and $\psi$ is a non-degenerate character of $N(E) \backslash N\left(\mathbb{A}_{E}\right)$. In the spectral development of (1), an orthonormal basis $\{\varphi\}$ for the space of cusp forms contributes

$$
\sum_{\{\varphi\}} \Pi^{H}(\rho(f) \varphi) \overline{W_{\varphi}(e)}
$$

where $W_{\varphi}(e)$ is the value at $e$ of the Fourier coefficient of $\varphi$ with respect to $\psi$. To evaluate the contribution of the continuous spectrum, we are led to truncate the continuous part of the kernel in the first variable and this leads to an integral involving the truncated periods $\Pi^{H}\left(\Lambda^{T} E\right)$ where $E$ is an Eisenstein series. Of course, it is at this stage in the development of the ordinary Arthur-Selberg trace formula that the inner products of truncated Eisenstein series appear. Unlike the Arthur-Selberg situation, the integral (1) itself is absolutely convergent. Nevertheless, truncation is needed to obtain an explicit formula. See [GJR] for an example where the formulas for $\Pi^{H}\left(\Lambda^{T} E\right)$ are used in this context. That work considers the case $G=G L(3)$ and compares (1) with the Kuznetzov trace formula for the group $G^{\prime}=U(3)$. The comparison is used in [FGJR] to characterize $H$-distinguished representations and to prove that a cuspidal $L$-packet on $U(3)$ (which is not a non-tempered A-packet) contains a generic element.

We now describe our results in greater detail. The first section deals with the meromorphic continuation of the integral of an exponential polynomial over a cone. This is used in Section IV to show that in the case $G=\operatorname{Res}_{E / F} H$ with $E / F$ quadratic, it is possible to define a regularized period $\Pi^{H}(\varphi)$ for automorphic forms whose exponents satisfy a certain mild restriction. The regularization is based on Arthur's truncation operators ([A2]) and their associated combinatorics. However, in the context of a pair $(G, H)$, there is more than one way to define the truncation. For example, one can apply Arthur's truncation operator $\Lambda^{T}$ relative to $G$ (cf. [LE], [LA2]) or one can restrict $\varphi$ and apply Arthur's truncation $\Lambda^{T}$ operator relative to $H$. While the former is the more natural of these two, we use a third, mixed truncation $\Lambda_{m}^{T}$, intermediate between them (in fact, this was used in [JL] in the $G L(2)$ case). This truncation appears to be best suited to the study of period integrals. The next step is to compute the period of a truncated Eisenstein series. In $\S 8$, we obtain a general formula for periods of the mixed truncation

$$
\int_{H(F) \backslash H(\mathbb{A})^{1}} \Lambda_{m}^{T} \varphi(h) d h
$$

in terms of the regularized periods of the constant terms of $\varphi$.

The last two sections of the article are devoted to more explicit results for $H=G L(n)_{/ F}$. In Section VI, we compute the regularized period $\Pi^{H}(E(g, \varphi, \lambda))$ for all cuspidal Eisenstein series (Theorem 23) and show, in particular, that it vanishes unless $E(g, \varphi, \lambda)$ is induced from cuspidal representation $\sigma_{1} \otimes \sigma_{2}$ of a parabolic subgroup of type $\left(\frac{n}{2}, \frac{n}{2}\right)$ with $\sigma_{1}^{*} \approx \overline{\sigma_{2}}$. We then obtain an explicit formula expressing $\Pi^{H}\left(\Lambda_{m}^{T} E(g, \varphi, \lambda)\right)$, for any cuspidal Eisenstein series, in terms of certain linear functionals $J(\xi, \varphi, \lambda)$ which we call intertwining periods. They are defined for $\lambda$ in 
a suitable cone by an absolutely convergent integral

$$
J(\xi, \varphi, \lambda)=\int_{H_{\eta}(\mathbb{A}) \backslash H(\mathbb{A})} \int_{M_{\eta}(F) \backslash M_{\eta}(\mathbb{A})^{1}} e^{\left\langle\lambda+\rho_{P}, H_{P_{E}}(\eta h)\right\rangle} \varphi(m \eta h) d m d h .
$$

Here $\xi, \eta \in G L_{n}(E)$ are elements such that $\eta \bar{\eta}^{-1}=\xi, H_{\eta}=H \cap \eta^{-1} P_{E} \eta$ where $P_{E}$ is the parabolic subgroup of $G$ from which the Eisenstein series is induced, and $M_{\eta}=$ $\eta H \eta^{-1} \cap M_{P_{E}}$ where $M_{P_{E}}$ is the Levi factor of $P_{E}$. The name "intertwining period" seems appropriate for several reasons. First, the map $\varphi \rightarrow J(\xi, \varphi, \lambda)$ is an $H(\mathbb{A})$ invariant functional on the induced representation $\operatorname{Ind}_{P}^{G} \sigma \otimes e^{\lambda}$ and so, by Frobenius reciprocity, defines an intertwining operator. Furthermore, the $J$-functionals have several properties in common with the standard intertwining operators $M(s, \lambda)$. Our explicit formula for the period integral

$$
\int_{H(F) \backslash H(\mathbb{A})^{1}} \Lambda_{m}^{T} E(h, \varphi, \lambda) d h
$$

is

$$
\sum_{(Q, s) \in \mathcal{G}(P, \sigma)} v_{Q} \frac{e^{\left\langle(s \lambda)_{Q}, T\right\rangle}}{\prod_{\alpha \in \Delta_{Q}}\left\langle(s \lambda)_{Q}, \alpha^{\vee}\right\rangle} J\left(\xi_{Q}, M(s, \lambda) \varphi,(s \lambda)_{P^{\prime}}^{Q}\right),
$$

where $Q$ and $s$ range over certain sets of parabolic subgroups and Weyl group elements, respectively, and $v_{Q}$ is a certain volume (see Section VIII for other unexplained notation). This formula is clearly analogous to Langlands' formula [A2] for the inner product of cuspidal Eisenstein series induced from parabolic subgroups $P$ and $P^{\prime}$, which expresses the inner product $\left(\Lambda^{T} E(\lambda, \varphi), \Lambda^{T} E(\mu, \psi)\right)$ as a sum

$$
\sum_{Q} \sum_{\substack{s \in \Omega(P, Q) \\ t \in \Omega\left(P^{\prime}, Q\right)}} \frac{e^{\langle s \lambda+t \mu, T\rangle}}{\prod_{\alpha \in \Delta_{Q}}\left\langle s \lambda+t \mu, \alpha^{\vee}\right\rangle}(M(s, \lambda) \varphi, M(t, \mu) \psi) .
$$

Like the standard intertwining operators, the $J$-functionals can be meromorphically continued and satisfy a set of functional equations. These are described in Section VII, again for the case $H=G L(n)_{/ F}$. The functional equations take the form

$$
J(\xi, \varphi, \lambda)=J\left(s \xi s^{-1}, M(s, \lambda) \varphi, s \lambda\right)
$$

for elements $s$ belonging to a certain subset of the Weyl group which we describe combinatorially in $\S 17$. For example, if $n=2$ and $\xi=\left(\begin{array}{ll}0 & 1 \\ 1 & 0\end{array}\right)$, then $H_{\eta}=T$ is a torus isomorphic to $E^{*}$ in $H$. In this case, the regularized period $\Pi^{H}(E(g, \varphi, \lambda))$ is equal to $J(\xi, \varphi, \lambda)$. For the choice $\varphi \equiv 1$,

$$
J(\xi, \varphi, \lambda)=\int_{T(F) \backslash G L_{2}\left(\mathbb{A}_{F}\right)} e^{\langle\lambda+1, H(\eta h)\rangle} d h
$$

and we have the following explicit formula:

$$
J(\xi, \varphi, \lambda)=\frac{1}{2} \operatorname{vol}(Z(\mathbb{A}) T(F) \backslash T(\mathbb{A})) \frac{L\left(\lambda, 1_{F}\right)}{L\left(\lambda+1, \omega_{E / F}\right)}
$$

where $\omega_{E / F}$ is the order two character attached to $E / F$ and $L\left(\lambda, 1_{F}\right)$ is the zetafunction of $F$ with archimedean Euler factors. The functional equation in this case reduces to

$$
J(\xi, \varphi, \lambda)=m(\xi, \lambda) J(\xi, \varphi,-\lambda)
$$


where $m(\xi, \lambda)=L\left(\lambda, 1_{E}\right) / L\left(\lambda+1,1_{E}\right)$. In $\S 20$ we show that the intertwining period factors as a product of local intertwining periods and we compute the local period in the unramified case in terms of Asai $L$-functions.

It is formula (3) which will be useful for applications to the relative trace formula. For example, if $H=G L(2)$, then (2) is equal to

$$
\begin{aligned}
& \frac{e^{\lambda T}}{\lambda} \int_{\mathbf{K}} \varphi(k) d k+\frac{e^{-\lambda T}}{-\lambda} \int_{\mathbf{K}} M(\xi, \lambda) \varphi(k) d k \\
& +\int_{T(F) \backslash G L_{2}\left(\mathbb{A}_{F}\right)} e^{\langle\lambda+1, H(\eta h)\rangle} \varphi(\eta h) d h
\end{aligned}
$$

for a suitable normalization of measures. Here $\xi$ is the non-trivial Weyl group element, $\eta \bar{\eta}^{-1}=\xi$, and $\mathbf{K}$ is a maximal compact subgroup of $G L_{2}\left(\mathbb{A}_{F}\right)$. This special case was first found by Jacquet and Lai.

It is possible to compute (2) using $\Lambda^{T}$ for $G$ in place of $\Lambda_{m}^{T}$. This calculation, involving multi-dimensional residues and contour shifts, follows the main lines of the traditional proof of Langlands inner product formula [A2], although it is substantially more complicated. We note that a similar analysis is attempted in [F3]. In the case $\left(G L(n)_{/ E}, G L(n)_{/ F}\right)$, one finds that for any automorphic form $\varphi$, the period integrals of $\Lambda_{m}^{T} \varphi$ and $\Lambda^{T} \varphi$ are the same.

The regularization of integrals is also useful in providing a more conceptual approach to the formulas for inner products of truncated Eisenstein series that occur in the Arthur-Selberg trace formula. In Section V, we describe the results one obtains by applying the above methods to the case of arbitrary $G$ and $\theta=i d$, so that $H=G$. The problem is to define a regularization of the integral

$$
\int_{G(F) \backslash G(\mathbb{A})^{1}} \varphi(g) d g .
$$

As in the case of period integrals, this is possible for automorphic forms $\varphi$ whose exponents satisfy a certain mild restriction. The regularized integral can then be used to derive a formula for the (convergent) integral

$$
\int_{G(F) \backslash G(\mathbb{A})^{1}} \Lambda^{T} \varphi(g) d g .
$$

These results can be extended to yield a formula for the inner product of truncated automorphic forms

$$
\begin{aligned}
& \int_{G(F) \backslash G(\mathbb{A})^{1}} \Lambda^{T} \varphi(g) \Lambda^{T} \psi(g) d g \\
= & \sum_{P \subset G}(-1)^{d(P)-d(G)} \int_{P(F) \backslash G(\mathbb{A})^{1}}^{*} \psi_{P}(g) \varphi_{P}(g) \hat{\tau}_{P}(H(g)-T) d g .
\end{aligned}
$$

The terms on the right are regularized inner products of the constant terms of $\varphi$ and $\psi$ and hence they are denoted with a star. This should be seen as a generalization of Langlands' formula for the inner product of truncated cuspidal Eisenstein series ([L], [A2]). In fact, Langlands' formula (4) follows directly from (6), the standard formula for the constant term of an Eisenstein series and Lemma 16. Thus (6) provides a conceptual proof of Langlands' formula. Note that (6) is a formula for the period of a truncated automorphic form on $G \times G$ relative to the period subgroup $G$ embedded diagonally. We omit the proofs in Section V because they are nearly identical, step-by-step, with those in Section IV. It is also possible to 
extend this method to give a new proof of Arthur's asymptotic formula [A4] for the inner product of truncated non-cuspidal Eisenstein series, where the main term is what is anticipated by the cuspidal case ([LP]).

Period integrals have been investigated from different points of view in recent years in a number of works by several authors, of which we mention the following sample: [F2], [F4], [FJ], [GRS], [JI], [JR], [JY], [MA], [SA]. A regularized integral for $G L(2)$ was developed by Zagier [Z], who gave a variety of applications, and was reformulated by Casselman $[\mathrm{C}]$. As mentioned above, our general version is based on the combinatorics of Arthur's truncation operators. We are grateful to J. Bernstein and W. Casselman for pointing out the relevance of regularization to our problem. We are also grateful to J. Bernstein for helpful conversations, and for providing the additional key insight that, in many cases, the regularized integral of an Eisenstein series vanishes for simple representation-theoretic reasons (Lemma $16)$.

\section{INTEGRALS OVER CONES}

Let $V$ be a real finite-dimensional vector space of dimension $n$. Let $V^{*}$ be the space of complex linear forms on $V$ and $V_{\mathbb{R}}^{*}$ the space of real linear forms. We denote by $S\left(V^{*}\right)$ the symmetric algebra of $V^{*}$, that is, the space of polynomial functions on $V$. Likewise we denote by $S(V)$ the symmetric algebra of $V \otimes \mathbb{C}$. An exponential polynomial function on $V$ is a function of the form:

$$
f(x)=\sum_{1 \leq i \leq r} e^{\left\langle\lambda_{i}, x\right\rangle} P_{i}(x)
$$

where the $\lambda_{i}$ are distinct elements of $V^{*}$ and the $P_{i}(x)$ are non-zero elements of $S\left(V^{*}\right)$. The $\lambda_{i}$ are then uniquely determined and called the exponents of $f$. We recall the proof of uniqueness.

For $X \in V$ we denote by $D_{X}$ the corresponding vector field:

$$
D_{X} g(x)=\left.\frac{d}{d t} g(x+t X)\right|_{t=0} .
$$

For $\lambda \in V^{*}$ we have:

$$
D_{X} e^{\langle\lambda, x\rangle}=\langle\lambda, X\rangle e^{\langle\lambda, x\rangle} .
$$

We can define the operators $D_{X}$ for $X \in S(V)$ by multiplicativity. Suppose that $P$ is in $S\left(V^{*}\right)$. There is an integer $d$ (total degree of $P$ ) such that for all $X \in V$ :

$$
D_{X}^{d} P=0 \text {. }
$$

Now let $g(x)=e^{\langle\lambda, x\rangle} P(x)$. Then

$$
\left(D_{X}-\langle\lambda, X\rangle\right) g(x)=e^{\langle\lambda, x\rangle} D_{X} P(x) .
$$

Thus

$$
\left(D_{X}-\langle\lambda, X\rangle\right)^{d} g(x)=0 .
$$

We will need the analogous fact for finite difference operators. Denote by $\Delta_{X}$ the finite difference operator defined by:

$$
\Delta_{X} f(x)=f(x+X)-f(x) .
$$

Then:

$$
\left(\Delta_{X}-\left(e^{\langle\lambda, X\rangle}-1\right)\right) e^{\langle\lambda, x\rangle} P(x)=e^{\langle\lambda, x\rangle} e^{\langle\lambda, X\rangle} \Delta_{X} P(x) .
$$


Thus if $d$ is the total degree of $P$, then

$$
\left(\Delta_{X}-\left(e^{\langle\lambda, X\rangle}-1\right)\right)^{d} e^{\langle\lambda, x\rangle} P(x)=0 .
$$

Now if $f$ has the form

$$
f(x)=\sum_{1 \leq i \leq r} e^{\left\langle\lambda_{i}, x\right\rangle} P_{i}(x),
$$

as above, choose $X \in V$ such that the numbers $\left\langle\lambda_{i}, X\right\rangle$ are distinct. By the Chinese remainder theorem, for each $i$, there exists a polynomial $Q_{i}$ in one variable such that

$$
Q_{i}\left(D_{X}\right) f(x)=e^{\left\langle\lambda_{i}, x\right\rangle} P_{i}(x) .
$$

Similarly, if the numbers $e^{\left\langle\lambda_{i}, X\right\rangle}$ are distinct there is for each $i$ a polynomial in one variable $R_{i}$ such that

$$
R_{i}\left(\Delta_{X}\right) f(x)=e^{\left\langle\lambda_{i}, x\right\rangle} P_{i}(x)
$$

This shows that the $\lambda_{i}$ are uniquely determined by $f$.

By a cone in $V$ we shall mean a closed subset of the form

$$
\mathcal{C}=\left\{x \in V:\left\langle\mu_{i}, x\right\rangle \geq 0\right\}
$$

where $\left\{\mu_{i}\right\}$ is a basis of $V_{\mathbb{R}}^{*}$. Let $e_{i}$ be the dual basis of $V$. We will say that $\lambda \in V^{*}$ is negative with respect to $\mathcal{C}$ if $\operatorname{Re}\left\langle\lambda, e_{j}\right\rangle<0$ for each $j=1, \ldots, n$. We will say that $\lambda$ is non-degenerate with respect to $\mathcal{C}$ if $\left\langle\lambda, e_{j}\right\rangle \neq 0$ for each $j=1, \ldots, n$. Our goal is to define the regularized integral of an exponential polynomial function over a cone.

Lemma 1. The function

$$
f(x)=\sum_{i} e^{\left\langle\lambda_{i}, x\right\rangle} P_{i}(x)
$$

is integrable over $\mathcal{C}$ if and only if $\lambda_{i}$ is negative with respect to $\mathcal{C}$ for all $i$.

Proof. The condition is clearly sufficient. Moreover it is necessary if $r=1$. In general, suppose that $f$ is integrable over $\mathcal{C}$. Choose $X$ such that the numbers $e^{\left\langle\lambda_{i}, X\right\rangle}$ are distinct, and, in addition, the numbers $\left\langle\mu_{j}, X\right\rangle$ are positive. Then $X+\mathcal{C}$ is contained in $\mathcal{C}$. It follows that the function $\Delta_{X} f$ is integrable over $\mathcal{C}$. Hence the functions $R_{i}\left(\Delta_{X}\right) f$ are also integrable over $\mathcal{C}$. Thus each term in the sum over $i$ is integrable over $\mathcal{C}$ and our assertion follows.

To define the regularized integral over $\mathcal{C}$ we study the integral

$$
I_{\mathcal{C}}(\lambda)(f):=\int_{\mathcal{C}} f(x) e^{-\langle\lambda, x\rangle} d x .
$$

The integral converges absolutely for $\lambda$ in the open set

$$
U=\left\{\lambda \in V^{*}: \operatorname{Re}\left\langle\lambda_{i}-\lambda, e_{j}\right\rangle<0 \text { for all } 1 \leq i \leq r ; 1 \leq j \leq n\right\} .
$$

We analytically continue this integral as follows. First, in the one-variable case, we have for $\operatorname{Re}(\lambda)>0$ and any polynomial $P$ in one variable:

$$
\int_{0}^{+\infty} e^{-\lambda x} P(x) d x=\sum_{m \geq 0} \frac{\left(D^{m} P\right)(0)}{\lambda^{m+1}} .
$$


Of course the sum on the right is finite. In general, fix an index $k$. Let $\mathcal{C}_{k}$ be the intersection of $\mathcal{C}$ and the hyperplane $V_{k}=\left\{x \mid\left\langle\mu_{k}, x\right\rangle=0\right\}$. In the integral we can write $x=\left\langle\mu_{k}, x\right\rangle e_{k}+y$ with $y \in V_{k}$ and then, for a suitable choice of the Haar measures:

$$
I_{\mathcal{C}}(\lambda)(f)=\sum_{1 \leq i \leq r} \sum_{m \geq 0} \frac{1}{\left\langle\lambda-\lambda_{i}, e_{k}\right\rangle^{m+1}} \int_{\mathcal{C}_{k}} e^{-\left\langle\lambda-\lambda_{i}, y\right\rangle}\left(D_{e_{k}}^{m}\right) P_{i}(y) d y .
$$

This formula gives the analytic continuation of $I_{\mathcal{C}}(\lambda)(f)$ to the tube domain $U_{k}$ defined by:

$$
\operatorname{Re}\left\langle\lambda_{i}-\lambda, e_{j}\right\rangle<0 \text { for } j \neq k, 1 \leq i \leq r .
$$

The analytic continuation is a meromorphic function with hyperplane singularities, the singular hyperplanes being:

$$
H_{k, i}=\left\{\lambda \mid\left\langle\lambda, e_{k}\right\rangle=\left\langle\lambda_{i}, e_{k}\right\rangle\right\}, 1 \leq i \leq r .
$$

Since this is true for all $1 \leq k \leq n$, the function $I_{\mathcal{C}}(\lambda)(f)$ has analytic continuation to $V^{*}$ by Hartogs' lemma. The continuation is a meromorphic function with hyperplane singularities. The singular hyperplanes are the hyperplanes $H_{i, k}$, $1 \leq k \leq n, 1 \leq i \leq r$. Of course, we could also use induction on $n$ and the above formula to obtain an explicit expression for the integral.

Lemma 2. Suppose that $f$ is absolutely integrable on $\mathcal{C}$. Then $I_{\mathcal{C}}(\lambda)(f)$ is holomorphic at 0 and $I_{\mathcal{C}}(0)(f)$ is the integral of $f$ over $\mathcal{C}$.

Proof. By the previous lemma, if $f$ is integrable over $\mathcal{C}$, then each exponent $\lambda_{i}$ is negative with respect to $\mathcal{C}$. Thus the domain of convergence $U$ of the integral $I(\lambda)(f)$ contains the point 0 and our assertion follows.

Lemma 3. The function $I_{\mathcal{C}}(\lambda)(f)$ is holomorphic at $\lambda=0$ if and only if for all $i$, $\lambda_{i}$ is non-degenerate with respect to $\mathcal{C}$, i.e., $\left\langle\lambda_{i}, e_{k}\right\rangle \neq 0$ for every pair $(i, k)$.

Proof. If the condition of the lemma is satisfied, then 0 does not belong to any of the hyperplanes $H_{i, k}$. Thus the function is holomorphic at 0 . To show conversely that the condition is necessary, it suffices to show that each hyperplane $H_{i, k}$ is indeed a singular hyperplane for the function $I_{\mathcal{C}}(\lambda)(f)$. Assume that some $H_{i_{0}, k}$ is not singular. Let $c=\left\langle\lambda_{i_{0}}, e_{k}\right\rangle$. Then

$$
\sum_{\left\{i \mid \lambda_{i}\left(e_{k}\right)=c\right\}} \sum_{m \geq 0} \frac{1}{\left\langle\lambda-\lambda_{i}, e_{k}\right\rangle^{m+1}} \int_{\mathcal{C}_{k}} e^{-\left\langle\lambda-\lambda_{i}, y\right\rangle}\left(D_{e_{k}}^{m}\right) P_{i}(y) d y
$$

must be identically zero for $\lambda \in U_{k}$. In turn this implies:

$$
\sum_{\left\{i \mid \lambda_{i}\left(e_{k}\right)=c\right\}} \int_{\mathcal{C}_{k}} e^{-\left\langle\lambda-\lambda_{i}, y\right\rangle}\left(D_{e_{k}}^{m}\right) P_{i}(y) d y=0
$$

for each $m$. By the Fourier inversion formula this implies:

$$
\sum_{\left\{i \mid\left\langle\lambda_{i}, e_{k}\right\rangle=c\right\}} e^{-\left\langle\lambda-\lambda_{i}, y\right\rangle}\left(D_{e_{k}}^{m}\right) P_{i}(y)=0
$$

for all $y \in V_{k}$ and all $m$. Note that in this formula the linear forms $\lambda_{i}$ have distinct restrictions to the hyperplane $V_{k}$. It follows that for each index $i$ with $\left\langle\lambda_{i}, e_{k}\right\rangle=c$ we have:

$$
\left(D_{e_{k}}^{m}\right) P_{i}(y)=0
$$


for each $m \geq 0$ and each $y \in V_{k}$. This relation implies $P_{i}=0$ for each $i$ with $\left\langle\lambda_{i}, e_{k}\right\rangle=c$. This is a contradiction and the lemma follows.

We observe that we can obtain the analytic continuation of the integral in a more direct way. This is useful if one wishes to study how the integral depends on parameters. In one variable we have the formula:

$$
\int_{0}^{\infty} e^{-\lambda x} D^{m} f(x) d x=\sum_{0 \leq u \leq m-1} \lambda^{m-1-u}\left(D^{u} f\right)(0)+\lambda^{m} \int_{0}^{\infty} e^{-\lambda x} f(x) d x .
$$

In general if $T$ is a polynomial in one variable and $T(X)=\sum_{i} t_{i} X^{i}$ we set:

$$
\tilde{T}(X, \lambda)=\sum_{i} t_{i} \sum_{0 \leq u \leq i-1} \lambda^{i-1-u} X^{u}
$$

Then

$$
\int_{0}^{\infty} e^{-\lambda x}(T(D) f)(x) d x=\tilde{T}(D, \lambda) f(0)+T(\lambda) \int_{0}^{\infty} e^{-\lambda x} f(x) d x .
$$

In particular, if $T(D) f=0$, then

$$
\int_{0}^{\infty} e^{-\lambda x} f(x) d x=-\frac{(\tilde{T}(D, \lambda) f)(0)}{T(\lambda)}
$$

This formula gives the analytic continuation of the integral. If $T(0) \neq 0$, then the integral is holomorphic at $\lambda=0$. If $T(0)=0$, then $T(X)=X S(X)$ where $S$ is another polynomial. The value of the numerator at $\lambda=0$ is $S(D) f(0)$. Thus 0 is a pole unless $S(D) f(0)=0$. Since $D S(D) f=0$ this is equivalent to $S(D) f=0$.

The generalization to higher dimensions is straightforward. Suppose that $f$ is an exponential polynomial function and for $k=1, \ldots, n$ there exists a polynomial $T_{k}$ in one variable such that

$$
T_{k}\left(D_{e_{k}}\right) f=0
$$

Then

$$
I_{\mathcal{C}}(\lambda)(f)=-\int_{\mathcal{C}_{k}} \frac{\left(\tilde{T}_{k}\left(D_{e_{k}},\left\langle\lambda, e_{k}\right\rangle\right) f\right)(y)}{T_{k}\left(\left\langle\lambda, e_{k}\right\rangle\right)} d y
$$

Using induction on $n$ we obtain:

$$
I_{\mathcal{C}}(\lambda)(f)=(-1)^{n} \frac{\left[\prod_{k} \tilde{T}_{k}\left(D_{e_{k}},\left\langle\lambda, e_{k}\right\rangle\right) f\right](0)}{\prod_{k} T_{k}\left(\left\langle\lambda, e_{k}\right\rangle\right)} .
$$

This can be used to give the analytic continuation of $I_{\mathcal{C}}(\lambda)(f)$. The singular hyperplanes have the form $\lambda\left(e_{k}\right)=z_{k}$ where $1 \leq k \leq n$ and $z_{k}$ is a root of the polynomial $T_{k}$. Thus if $T_{k}(0) \neq 0$ for $1 \leq k \leq n$, then $I_{\mathcal{C}}(\lambda)(f)$ is holomorphic at $\lambda=0$.

Note that if 0 is a root of $T_{k}$, then $T_{k}(X)=X S_{k}(X)$ where $S_{k}$ is another polynomial. As in the one-variable case the hyperplane $\lambda\left(e_{k}\right)=0$ is then singular unless $S_{k}\left(D_{e_{k}}\right) f=0$.

Although it is not needed in the sequel, we remark that with this approach, it is easy to see that under appropriate assumptions the value of the integral will depend holomorphically on a parameter if $f$ does. To that end, consider the following situation. Let $A$ be a finitely generated subalgebra of $S(V)$ (containing 1). Suppose that $S(V)$ is finitely generated as an $A$-module. Next suppose that $\Omega \subset \mathbb{C}^{q}$ is a 
connected open set and for each $s \in \Omega$ we are given a character $\chi_{s}$ of $A$, that is, a homomorphism $\chi_{s}: A \rightarrow \mathbb{C}$, depending holomorphically on $s$. This means that the values of $\chi_{s}$ on a set of generators of $A$ are holomorphic functions of $s$ in $\Omega$. Next, let $f_{s}(x)$ be a smooth function on $V \times \Omega$. We assume it is holomorphic in $s$ and satisfies

$$
D_{X} f_{s}(x)=\chi_{s}(X) f_{s}(x)
$$

for all $X \in A$. For each $k$ with $1 \leq k \leq n$, let $B_{k}$ be the algebra generated by $e_{k}$ and $A$. Because $A$ is Noetherian it is a finitely generated $A$-module. It follows that there is a polynomial $T_{k} \in A[X]$, whose coefficient of the term of highest degree is 1 , such that $T_{k}\left(e_{k}\right)=0$. The polynomial $T_{k}$ has the form:

$$
T_{k}(X)=\sum a_{i} X^{i}
$$

with $a_{i} \in A$. We set

$$
T_{k}(X ; s)=\sum \chi_{s}\left(a_{i}\right) X^{i}
$$

For each $s \in \Omega$ this is a polynomial with complex coefficients and

$$
T_{k}\left(D_{e_{k}} ; s\right) f_{s}=0 .
$$

If $\Omega^{\prime}$ is a relatively compact open subset of $\Omega$, one can use this system of differential equations to obtain a majorization of $f_{s}$ on $C$, uniform for $s \in \Omega^{\prime}$, and show that there is an open set $U$ defined by inequalities of the form $\operatorname{Re}\left(\left\langle\lambda, e_{i}\right\rangle\right)>>0$ such that the integral $I_{\mathcal{C}}(\lambda)\left(f_{s}\right)$ converges uniformly on compact subsets of $U \times \Omega^{\prime}$. To the polynomial $T_{k}(X ; s)$ we associate as before the polynomial $\tilde{T}_{k}(X, \lambda ; s)$. Then the formula

$$
I_{\mathcal{C}}(\lambda)\left(f_{s}\right)=(-1)^{n} \frac{\left[\prod_{k} \tilde{T}_{k}\left(D_{e_{k}},\left\langle\lambda, e_{k}\right\rangle ; s\right) f_{s}\right](0)}{\prod_{k} T_{k}\left(\left\langle\lambda, e_{k}\right\rangle ; s\right)}
$$

gives the analytic continuation of the integral as a meromorphic function of $(\lambda, s)$. We have the following more precise result.

Proposition 4. With the previous notations assume that there is a point $s_{0} \in \Omega$ such that any character $\chi$ of $S(V)$ which extends $\chi_{s_{0}}$ takes a non-zero value on the vectors $e_{k}$. Then $I_{\mathcal{C}}(\lambda)\left(f_{s}\right)$ is holomorphic at $\left(0, s_{0}\right)$.

Proof. Since $S(V)$ is integral over $A$, every character of $A$ extends (in finitely many ways) to $S(V)$ (by the going up theorem). Consider the set $I_{k}$ of polynomials $P \in A[X]$ such that $P\left(e_{k}\right)=0$. It is a (finitely generated) ideal. Let $\left\{T_{\alpha}\right\}$ be a set of generators of $I_{k}$. An extension $\chi$ of $\chi_{s_{0}}$ to $B_{k}$ is determined by a complex number $z$ such that $T_{\alpha}\left(z ; s_{0}\right)=0$ for all $\alpha$ and then $\chi\left(e_{k}\right)=z$. Our assumption is that such an extension does not take the value 0 on $e_{k}$. Thus there must be at least one index $\alpha$ such that $T_{\alpha}\left(0 ; s_{0}\right) \neq 0$. Re-label such a polynomial $T_{k}(X ; s)$. We thus have

$$
T_{k}\left(D_{e_{k}} ; s\right) f_{s}=0
$$

for all $s$ and $T_{k}\left(0, s_{0}\right) \neq 0$. Again, the formula

$$
I_{\mathcal{C}}(\lambda)\left(f_{s}\right)=(-1)^{n} \frac{\left[\prod_{k} \tilde{T}_{k}\left(D_{e_{k}},\left\langle\lambda, e_{k}\right\rangle ; s\right) f_{s}\right](0)}{\prod_{k} T_{k}\left(\left\langle\lambda, e_{k}\right\rangle ; s\right)}
$$

does show that $I_{\mathcal{C}}(\lambda)\left(f_{s}\right)$ is holomorphic at $\left(0, s_{0}\right)$. 
We remark that the coefficient $a_{k}$ of the term of highest degree of $T_{k}$ need not be 1 . Moreover we may have $\chi_{s_{0}}\left(a_{k}\right)=0$.

After these preliminaries we define the regularized integral. Denote the characteristic function of a set $\mathcal{Y}$ by $\tau^{\mathcal{Y}}$. Suppose that $\mathcal{C}$ is the cone defined relative to a basis $\left\{\mu_{i}\right\}$ of $V_{\mathbb{R}}^{*}$ as in (8) and let $\left\{e_{i}\right\}$ denote the dual basis. Let $T \in V$. As before, let

$$
f(x)=\sum_{1 \leq i \leq r} e^{\left\langle\lambda_{i}, x\right\rangle} P_{i}(x)
$$

be an exponential polynomial. For $\lambda \in V^{*}$ such that $\lambda_{i}-\lambda$ is negative with respect to $\mathcal{C}$ for all $i=1, \ldots, r$, set

$$
\begin{aligned}
\widehat{f}(\lambda ; \mathcal{C}, T) & =\int_{V} f(x) \tau^{\mathcal{C}}(x-T) e^{-\langle\lambda, x\rangle} d x \\
& =\sum_{1 \leq i \leq r} \int_{V} P_{i}(x) \tau^{\mathcal{C}}(x-T) e^{-\left\langle\lambda-\lambda_{i}, x\right\rangle} d x \\
& =\sum_{1 \leq i \leq r} e^{\left\langle\lambda_{i}-\lambda, T\right\rangle} I_{\mathcal{C}}(\lambda)\left(P_{i}(\bullet+T) e^{\left\langle\lambda_{i}, \bullet\right\rangle}\right) .
\end{aligned}
$$

The integrals are absolutely convergent and, as we have seen, $\widehat{f}(\lambda ; \mathcal{C}, T)$ extends a meromorphic function on $V^{*}$. We will say that the function $f(x) \tau^{\mathcal{C}}(x-T)$ is \#-integrable if $\widehat{f}(\lambda ; \mathcal{C}, T)$ is regular at $\lambda=0$. In this case, we define the \#-integral

$$
\int_{V}^{\#} f(x) \tau^{\mathcal{C}}(x-T) d x
$$

to be the value $\widehat{f}(0 ; \mathcal{C}, T)$. Otherwise, we say that the \#-integral does not exist. By Lemma 3 , the \#-integral exists if and only if each exponent $\lambda_{j}$ is non-degenerate with respect to $\mathcal{C}$.

Suppose that $V=W_{1} \oplus W_{2}$ is a decomposition of $V$ as a direct sum, and let $\mathcal{C}_{j}$ be a cone in $W_{j}$. Write $T=T_{1}+T_{2}$ and write $x=w_{1}+w_{2}$ relative to $V=W_{1} \oplus W_{2}$. If the exponents $\lambda_{j}$ of $f$ are non-degenerate with respect to $\mathcal{C}=\mathcal{C}_{1} \oplus \mathcal{C}_{2}$, then the function

$$
w_{2} \longrightarrow \int_{W_{1}}^{\#} f\left(w_{1}+w_{2}\right) \tau_{1}^{\mathcal{C}}\left(w_{1}-T_{1}\right) d w_{1}
$$

is defined and is an exponential polynomial, and it follows by analytic continuation that

is equal to

$$
\int_{V}^{\#} f(x) \tau^{\mathcal{C}}(x-T) d x
$$

$$
\int_{W_{2}}^{\#}\left(\int_{W_{1}}^{\#} f\left(w_{1}+w_{2}\right) \tau_{1}^{\mathcal{C}}\left(w_{1}-T_{1}\right) d w_{1}\right) \tau_{2}^{\mathcal{C}}\left(w_{2}-T_{2}\right) d w_{2} .
$$

Furthermore, we have

Lemma 5. Let $f$ be as in (9). Then the function

$$
T \longrightarrow \int_{V}^{\#} f(x) \tau^{\mathcal{C}}(x-T) d x
$$

is an exponential polynomial with the same exponents as $f$. 
Proof. It is clear from (10) that $\widehat{f}(\lambda ; \mathcal{C}, T)$ is an exponential polynomial as a function of $T$ with the same exponents as $f$. Hence the same is true of its value at $\lambda=0$.

The domain of the \#-integral can be extended in various ways. Without addressing this general question, we shall consider \#-integrals of the following slightly more general type of function which will be adequate for our later needs. Suppose that $V=W_{1} \oplus W_{2}$ is a decomposition of $V$ as a direct sum. Let $g(x)$ be a compactly supported function on $W_{1}$ and let $\mathcal{C}_{2} \subset W_{2}$ be a cone in $W_{2}$. Writing $T=T_{1}+T_{2}$ and $x=w_{1}+w_{2}$ as above, we consider functions of the form

$$
g\left(w_{1}-T_{1}\right) \tau_{2}^{\mathcal{C}}\left(w_{2}-T_{2}\right)
$$

which, for convenience, we will call functions of type (C).

It is clear that the integral

$$
\widehat{F}(\lambda)=\int_{V} f(x) g\left(w_{1}-T_{1}\right) \tau_{2}^{\mathcal{C}}\left(w_{2}-T_{2}\right) e^{-\langle\lambda, x\rangle} d x
$$

converges absolutely for an open set of $\lambda$ whose restriction to $W_{2}$ is negative with respect to $\mathcal{C}_{2}$. Furthermore, $\widehat{F}(\lambda)$ has a meromorphic continuation to $V^{*}$. As before, we say that $f(x) g\left(x-T_{1}\right) \tau_{2}^{\mathcal{C}}\left(x-T_{2}\right)$ is \#-integrable if $\widehat{F}(\lambda)$ is regular at $\lambda=0$. If so, we denote the value $\widehat{F}(0)$ by

$$
\int_{V}^{\#} f(x) g\left(w_{1}-T_{1}\right) \tau_{2}^{\mathcal{C}}\left(w_{2}-T_{2}\right) d x
$$

Note that the function

$$
w_{2} \longrightarrow \int_{W_{1}} f\left(w_{1}+w_{2}\right) g\left(w_{1}-T_{1}\right) d w_{1}
$$

is an exponential polynomial on $W_{2}$ and (13) is equal to the iterated integral

$$
\int_{W_{2}}^{\#}\left(\int_{W_{1}} f\left(w_{1}+w_{2}\right) g\left(w_{1}-T_{1}\right) d w_{1}\right) \tau_{2}^{\mathcal{C}}\left(w_{2}-T_{2}\right) d w_{2} .
$$

In the next lemma, for $i=1, \ldots, r$ let $V=W_{i 1} \oplus W_{i 2}$ be a direct sum decomposition and let $\mathcal{C}_{i 2}$ be a cone in $W_{i 2}$. Let $g_{i}$ be a compactly supported function on $W_{i 1}$ and set $G_{i}\left(w_{1}+w_{2}\right)=g_{i}\left(w_{1}\right) \tau_{i 2}^{\mathcal{C}}\left(w_{2}\right)$ for $w_{j} \in W_{i j}$.

Lemma 6. Let $\mathcal{C}$ and $\mathcal{C}^{*}$ be cones in $V$. Assume that $\mathcal{C}_{i 2}, \mathcal{C} \subset \mathcal{C}^{*}$ for all $i$. Assume further that

$$
\tau^{\mathcal{C}}(x)=\sum_{i=1}^{r} a_{i} G_{i}(x)
$$

for some constants $a_{i}$. Let $f$ be as in (9) and assume that each of the integrals

$$
\int_{V}^{\#} f(x) G_{i}(x-T) d x
$$

exists for $i=1, \ldots, r$. Then $f(x) \tau^{\mathcal{C}}(x-T)$ is \#-integrable and

$$
\int_{V}^{\#} f(x) \tau^{\mathcal{C}}(x-T) d x=\sum a_{i} \int_{V}^{\#} f(x) G_{i}(x-T) d x .
$$


Proof. In the open set of $\lambda$ such that $\lambda_{j}-\lambda$ is negative with respect to $\mathcal{C}^{*}$ for all $j$, we have an equality of absolutely convergent integrals

$$
\int_{V} f(x) \tau^{\mathcal{C}}(x) e^{-\langle\lambda, x\rangle} d x=\sum a_{i} \int_{V} f(x) G_{i}(x-T) e^{-\langle\lambda, x\rangle} d x .
$$

The assertion now follows by analytic continuation.

For future reference, we note the explicit formula

$$
\int_{V}^{\#} e^{\langle\lambda, x\rangle} \tau^{\mathcal{C}}(x-T) d x=(-1)^{n} v\left(e_{1}, \ldots, e_{n}\right) \frac{e^{\langle\lambda, T\rangle}}{\prod_{j=1}^{n}\left\langle\lambda, e_{j}\right\rangle}
$$

where, as above,

$$
\mathcal{C}=\left\{\sum_{j=1}^{n} a_{j} e_{j}: a_{j} \geq 0\right\}
$$

and $v\left(e_{1}, \ldots, e_{n}\right)$ is the volume of the parallelepiped $\left\{\sum_{j=1}^{n} a_{j} e_{j}: 0 \leq a_{j} \leq 1\right\}$. In the case $V=\mathbb{R}$ we have

$$
\int_{T}^{\infty} e^{\lambda t} d t=-\frac{e^{\lambda T}}{\lambda}
$$

and hence for any cone $\mathcal{C} \subset \mathbb{R}$,

$$
\int_{V}^{\#} e^{\langle\lambda, x\rangle}\left(1-\tau^{\mathcal{C}}(x-T)\right) d x=-\int_{V}^{\#} e^{\langle\lambda, x\rangle} \tau^{\mathcal{C}}(x-T) d x
$$

since $1-\tau^{C}$ is the characteristic function of the cone $-\mathcal{C}$.

\section{Automorphic FORMS AND TRUNCATION}

Let $G$ be a connected, reductive algebraic group over a number field $F$ with adele ring $\mathbb{A}$. In this section, we fix some notation and recall some definitions and results connected with Arthur's truncation operators.

1. Roots, coroots, etc. Fix a minimal $F$-parabolic subgroup $P_{0}$ of $G$ and a Levi decomposition $P_{0}=M_{0} N_{0}$. An $F$-parabolic subgroup $P$ is called standard if it contains $P_{0}$. If $P$ is standard, we write $M_{P}$ for the unique Levi factor of $P$ containing $M_{0}$ and $N_{P}$ for the unipotent radical of $P$. A Levi factor of the form $M_{P}$ will be called a standard Levi subgroup. Since we deal only with standard subgroups, we shall usually drop the word standard and use the term parabolic subgroup or Levi subgroup to denote a standard F-parabolic subgroup or standard Levi subgroup.

We now recall some standard definitions and notations ([A1], $[\mathrm{LR}])$. Let $P$ be a parabolic subgroup. We write $T_{P}$ for the maximal split torus in the center of $M_{P}$, and $T_{P}^{\prime}$ for the maximal quotient split torus of $M_{P}$. We set

$$
\widetilde{\mathfrak{A}}_{P}=X_{*}\left(T_{P}\right) \otimes \mathbb{R}=X_{*}\left(T_{P}^{\prime}\right) \otimes \mathbb{R},
$$

where $X_{*}(T)$ is the lattice of 1-parametric subgroups in a torus (the two vector spaces on the right are canonically isomorphic), and set

$$
d(P)=\operatorname{dim} \widetilde{\mathfrak{A}}_{P} .
$$


The two descriptions of $\widetilde{\mathfrak{A}}_{P}$ show that if $Q \subset P$ is a parabolic subgroup contained in $P$, then there is a canonical injection $\widetilde{\mathfrak{A}}_{P} \longrightarrow \widetilde{\mathfrak{A}}_{Q}$ and surjection $\widetilde{\mathfrak{A}}_{Q} \longrightarrow \widetilde{\mathfrak{A}}_{P}$. We obtain canonical decomposition

$$
\widetilde{\mathfrak{A}}_{Q}=\widetilde{\mathfrak{A}}_{Q}^{P} \oplus \widetilde{\mathfrak{A}}_{P} .
$$

In particular, $\widetilde{\mathfrak{A}}_{G}$ is a summand of $\widetilde{\mathfrak{A}}_{P}$ for all $P$. Set $\mathfrak{A}_{P}=\widetilde{\mathfrak{A}}_{P} / \widetilde{\mathfrak{A}}_{G}$ and $\mathfrak{A}_{Q}^{P}=\widetilde{\mathfrak{A}}_{P}^{Q} / \widetilde{\mathfrak{A}}_{G}$. Then we have

$$
\mathfrak{A}_{Q}=\mathfrak{A}_{Q}^{P} \oplus \mathfrak{A}_{P} .
$$

In particular, $\mathfrak{A}_{P}$ is canonically identified as a subspace of $\mathfrak{A}_{Q}$. Set $\mathfrak{A}_{0}=\mathfrak{A}_{P_{0}}$ and $\mathfrak{A}_{0}^{P}=\mathfrak{A}_{P_{0}}^{P}$. Then we also have

$$
\mathfrak{A}_{0}=\mathfrak{A}_{0}^{P} \oplus \mathfrak{A}_{P}
$$

for all $P$. We write $\mathfrak{A}_{0}^{*}, \mathfrak{A}_{P}^{*}$, etc. for the dual spaces (over $\mathbb{R}$ ).

We now define the standard bases of the above spaces and their duals. Let $\Delta_{0}$ and $\widehat{\Delta}_{0}$ be the subsets of simple roots and simple weights in $\mathfrak{A}_{0}^{*}$, respectively. We write $\Delta_{0}^{\vee}$ for the basis of $\mathfrak{A}_{0}$ dual to $\widehat{\Delta}_{0}$, and $\widehat{\Delta}_{0}^{\vee}$ for the basis of $\mathfrak{A}_{0}$ dual to $\Delta_{0}$. Thus, $\Delta_{0}^{\vee}$ is the set of coroots and $\widehat{\Delta}_{0}^{\vee}$ is the set of coweights.

For every $P$, let $\Delta_{P} \subset \mathfrak{A}_{0}^{*}$ be the set of non-trivial restrictions of elements of $\Delta_{0}$ to $\mathfrak{A}_{P}$. For each $\alpha \in \Delta_{P}$, let $\alpha^{\vee}$ be the projection of $\beta^{\vee}$ to $\mathfrak{A}_{P}$, where $\beta$ is the root in $\Delta_{0}$ whose restriction to $\mathfrak{A}_{P}$ is $\alpha$. Set $\Delta_{P}^{\vee}=\left\{\alpha^{\vee}: \alpha \in \Delta_{P}\right\}$. Then we may also define their dual bases. Namely, we denote the dual basis of $\Delta_{P}$ by $\widehat{\Delta}_{P}^{\vee}$ and the dual basis of $\Delta_{P}^{\vee}$ by $\widehat{\Delta}_{P}$.

If $Q \subset P$, we write $\Delta_{Q}^{P}$ to denote the subset of $\alpha \in \Delta_{Q}$ appearing in the action of $T_{Q}$ in the unipotent radical of $Q \cap M_{P}$. Then $\mathfrak{A}_{P}$ is the subspace of $\mathfrak{A}_{Q}$ annihilated by $\Delta_{Q}^{P}$. Let $\left(\Delta_{Q}^{P}\right)^{\vee}=\left\{\alpha^{\vee}: \alpha \in \Delta_{Q}^{P}\right\}$. We then define $\left(\widehat{\Delta}^{\vee}\right)_{Q}^{P}$ and $\widehat{\Delta}_{Q}^{P}$ to be the bases dual to $\Delta_{Q}^{P}$ and $\left(\Delta_{Q}^{P}\right)^{\vee}$, respectively.

2. Inversion formulas. For convenience, we give an abstract formulation of some inversion relations employed in Arthur's work. Suppose that $\left\{\tau_{P}^{Q}\right\},\left\{\hat{\tau}_{P}^{Q}\right\}$ are sets of constants indexed by pairs of parabolic subgroups $P \subset Q$ satisfying the relations

$$
\sum_{Q \subset R \subset P}(-1)^{d(R)-d(P)} \tau_{Q}^{R} \hat{\tau}_{R}^{P}=\delta_{Q P}
$$

for any $P \subset Q$.

This set of relations implies (and is equivalent to) the set of relations

$$
\sum_{Q \subset R \subset P}(-1)^{d(Q)-d(R)} \hat{\tau}_{Q}^{R} \tau_{R}^{P}=\delta_{Q P}
$$

Indeed,

$$
\begin{aligned}
& \sum_{Q \subset R \subset P}(-1)^{d(Q)-d(R)} \hat{\tau}_{Q}^{R} \tau_{R}^{P} \\
= & \sum_{Q \subset R \subset R^{\prime} \subset P}(-1)^{d(Q)-d(R)} \hat{\tau}_{Q}^{R} \delta_{R R^{\prime}} \tau_{R^{\prime}}^{P} \\
= & \sum_{Q \subset R \subset S \subset R^{\prime} \subset P}(-1)^{d(Q)-d(R)+d(S)-d\left(R^{\prime}\right)} \hat{\tau}_{Q}^{R} \tau_{R}^{S} \hat{\tau}_{S}^{R^{\prime}} \tau_{R^{\prime}}^{P} \\
= & \sum_{Q \subset R \subset S \subset R^{\prime} \subset P}(-1)^{d(Q)-d(R)+d(S)-d\left(R^{\prime}\right)} \hat{\tau}_{Q}^{R} \tau_{R}^{S} \hat{\tau}_{S}^{R^{\prime}} \tau_{R^{\prime}}^{P} .
\end{aligned}
$$


For fixed $S$, the sum over $R$ vanishes unless $S=Q$ and the sum over $R^{\prime}$ vanishes unless $S=P$. Hence the sum vanishes unless $Q=P$, in which case it equals $\hat{\tau}_{Q}^{Q} \tau_{Q}^{P}=1$.

Given arbitrary sets of constants $\left\{\alpha_{Q}^{P}\right\}$ and $\left\{\hat{\alpha}_{Q}^{P}\right\}$, define

$$
\begin{aligned}
& \beta_{Q}^{P}=\sum_{Q \subset R \subset P}(-1)^{d(R)-d(P)} \tau_{Q}^{R} \hat{\alpha}_{R}^{P}, \\
& \hat{\beta}_{Q}^{P}=\sum_{Q \subset S \subset P}(-1)^{d(Q)-d(S)} \alpha_{Q}^{S} \hat{\tau}_{S}^{P} .
\end{aligned}
$$

The following lemma is easily verified.

Lemma 7. The following relations hold:

1. $\hat{\alpha}_{Q}^{P}=\sum_{Q \subset R \subset P}(-1)^{d(R)-d(P)} \hat{\tau}_{Q}^{R} \beta_{R}^{P}$.

2. $\alpha_{Q}^{P}=\sum_{Q \subset R \subset P}(-1)^{d(Q)-d(R)} \hat{\beta}_{Q}^{R} \tau_{R}^{P}$.

3. If the constants $\left\{\alpha_{Q}^{P}\right\}$ and $\left\{\hat{\alpha}_{Q}^{P}\right\}$ satisfy

$$
\sum_{Q \subset R \subset P}(-1)^{d(Q)-d(R)} \hat{\alpha}_{Q}^{R} \alpha_{R}^{P}=\delta_{Q P}
$$

then

$$
\sum_{Q \subset R \subset P}(-1)^{d(R)-d(P)} \beta_{Q}^{R} \hat{\beta}_{R}^{P}=\delta_{Q P} .
$$

3. The standard characteristic functions. We now extend the linear functionals in $\Delta_{Q}^{P}$ and $\hat{\Delta}_{Q}^{P}$ to elements of the dual space $\mathfrak{A}_{0}^{*}$ by means of the canonical projection from $\mathfrak{A}_{0}$ to $\mathfrak{A}_{Q}^{P}$ given by the decomposition $\mathfrak{A}_{0}=A_{0}^{Q} \oplus A_{Q}^{P} \oplus A_{P}$. Let $\tau_{Q}^{P}$ be the characteristic function of the subset

$$
\left\{H \in \mathfrak{A}_{0}:\langle\alpha, H\rangle>0 \text { for all } \alpha \in \Delta_{Q}^{P}\right\}
$$

and let $\hat{\tau}_{Q}^{P}$ be the characteristic function of the subset

$$
\left\{H \in \mathfrak{A}_{0}:\langle\varpi, H\rangle>0 \text { for all } \varpi \in \hat{\Delta}_{Q}^{P}\right\} .
$$

We recall that $\hat{\tau}_{Q}^{P} \geq \tau_{Q}^{P}$. The following is a special case of Langlands' combinatorial lemma [A1].

Langlands' Lemma. If $Q \subset P$ are parabolic subgroups, then for all $H \in \mathfrak{A}_{0}$ we have

$$
\sum_{Q \subset R \subset P}(-1)^{d(R)-d(P)} \tau_{Q}^{R}(H) \hat{\tau}_{R}^{P}(H)=\delta_{Q P}
$$

and

$$
\sum_{Q \subset R \subset P}(-1)^{d(Q)-d(R)} \widehat{\tau}_{Q}^{R}(H) \tau_{R}^{P}(H)=\delta_{Q P} .
$$

For any pair of parabolic subgroups $P \subset Q$ we define functions $\Gamma_{P}^{Q}(H, X)$ and $\hat{\Gamma}_{P}^{Q}(H, X)$ for $H, X \in \mathfrak{A}_{0}$ by the formulas

$$
\begin{aligned}
& \Gamma_{Q}^{P}(H, X)=\sum_{Q \subset R \subset P}(-1)^{d(R)-d(P)} \tau_{Q}^{R}(H) \hat{\tau}_{R}^{P}(H-X), \\
& \hat{\Gamma}_{Q}^{P}(H, X)=\sum_{Q \subset S \subset P}(-1)^{d(Q)-d(S)} \tau_{Q}^{S}(H-X) \hat{\tau}_{S}^{P}(H) .
\end{aligned}
$$


These functions depend only on the projections of $H$ and $X$ onto $\mathfrak{A}_{Q}^{P}$. The function $\Gamma_{P}^{G}$ appears in [A3] and we follow $[\mathrm{LR}]$ in defining $\Gamma_{Q}^{P}, \hat{\Gamma}_{Q}^{P}$ for general $Q \subset P$. By Langlands' Lemma and Lemma 7, we obtain the formulas:

$$
\begin{aligned}
& \hat{\tau}_{Q}^{P}(H-X)=\sum_{Q \subset R \subset P}(-1)^{d(R)-d(P)} \hat{\tau}_{Q}^{R}(H) \Gamma_{R}^{P}(H, X), \\
& \tau_{Q}^{P}(H-X)=\sum_{Q \subset R \subset P}(-1)^{d(Q)-d(R)} \hat{\Gamma}_{Q}^{R}(H, X) \tau_{R}^{P}(H),
\end{aligned}
$$

and also

$$
\begin{aligned}
& \sum_{\substack{P \\
S \subset P \subset T}}(-1)^{d(P)-d(T)} \Gamma_{S}^{P}(H, X) \hat{\Gamma}_{P}^{T}(H, X)=\delta_{S T}, \\
& \sum_{S \subset P \subset T}(-1)^{d(S)-d(P)} \hat{\Gamma}_{S}^{P}(H, X) \Gamma_{P}^{T}(H, X)=\delta_{S T} .
\end{aligned}
$$

Finally, the following relation follows from the definitions:

$$
\hat{\Gamma}_{Q}^{P}(H, X)=(-1)^{d(Q)-d(R)} \Gamma_{Q}^{P}(H-X,-X),
$$

and therefore

$$
\tau_{Q}^{P}(H-X)=\sum_{Q \subset R \subset P} \Gamma_{Q}^{R}(H-X,-X) \tau_{R}^{P}(H) .
$$

4. Automorphic forms. We shall often write $G$ for $G(F), P$ for $P(F)$, etc., when there is no risk of confusion. To define automorphic forms on $G$, let $\mathcal{Z}$ be the center of the complexified universal enveloping algebra of $G_{\infty}$ and fix a good maximal compact subgroup $\mathbf{K} \subset G(\mathbb{A})$. Then the Iwasawa decomposition $G(\mathbb{A})=N_{P}(\mathbb{A}) M_{P}(\mathbb{A}) \mathbf{K}$ holds. For any $g \in G(\mathbb{A})$, we will say that $g=n m k$ is an Iwasawa decomposition relative to $P$ if $n \in N_{P}(\mathbb{A}), m \in M_{P}(\mathbb{A})$, and $k \in \mathbf{K}$.

A function

$$
\varphi: G \backslash G(\mathbb{A}) \longrightarrow C
$$

is called an automorphic form if

1. $\varphi$ is smooth and of moderate growth,

2. $\varphi$ is right $\mathbf{K}$-finite,

3. $\varphi$ is $\mathcal{Z}$-finite.

Let $\mathcal{A}(G)$ be the space of automorphic forms on $G \backslash G(\mathbb{A})$.

We also define the space $\mathcal{A}_{P}(G)$ for any parabolic subgroup $P=M N$. This is the space of smooth, right $\mathbf{K}$-finite functions

$$
\varphi: N(\mathbb{A}) M \backslash G(\mathbb{A}) \longrightarrow C
$$

such that for all $k \in \mathbf{K}$, the function $m \longrightarrow \varphi(m k)$ is an automorphic form on $M(\mathbb{A})$. For $\varphi \in \mathcal{A}_{P}(G)$ and any parabolic subgroup $Q \subset P$, the constant term is defined in the standard way:

$$
\varphi_{Q}(g)=\int_{N_{Q} \backslash N_{Q}(\mathbb{A})} \varphi(n g) d n .
$$

The map $\varphi \longmapsto \varphi_{Q}$ sends $\mathcal{A}_{P}(G)$ to $\mathcal{A}_{Q}(G)$.

For each parabolic subgroup $P$, we have the map

$$
H_{P}: G(\mathbb{A}) \longrightarrow \widetilde{\mathfrak{A}}_{P}
$$


characterized by the following two conditions: (1) $|\chi|(m)=e^{\left\langle\chi, H_{P}(m)\right\rangle}$ for all $m \in$ $M(\mathbb{A})$ and rational characters $\chi \in X^{*}\left(M_{P}\right)$, and $(2) H_{P}(n m k)=H_{P}(m)$ for all $n \in N(\mathbb{A}), m \in M(\mathbb{A})$, and $k \in \mathbf{K}$. We write $H(g)$ for $H_{P_{0}}(g)$. Then $H_{P}(g)$ is the projection of $H(g)$ onto $\widetilde{\mathfrak{A}}_{P}$. The kernel of the restriction of $H_{P}$ to $M(\mathbb{A})$ is denoted $M(\mathbb{A})^{1}$.

Set $F_{\infty}=F \otimes_{\mathbb{Q}} \mathbb{R}$. We have an isomorphism $\left(F_{\infty}^{*}\right)^{\ell} \simeq T_{P}\left(F_{\infty}\right)$ for some $\ell$. We may embed $\mathbb{R} \hookrightarrow F_{\infty}$ via $x \longrightarrow 1 \otimes x$ and thus view $\mathbb{R}_{+}^{*}$ as a subgroup of $F_{\infty}^{*}$ in a canonical way. Let $A_{P}$ for $P \neq G$ and $A_{G}$ denote the intersections of the image of $\left(\mathbb{R}_{+}^{*}\right)^{\ell}$ in $T_{P}\left(F_{\infty}\right)$ with $G(\mathbb{A})^{1}$ and $Z(\mathbb{A})$, respectively. The map $H_{P}$ induces an isomorphism of $A_{P}$ onto $\mathfrak{A}_{P}$ and we have $M(\mathbb{A})=A_{G} \times A_{P} \times M(\mathbb{A})^{1}$. For $X \in \mathfrak{A}_{P}$, we write $e^{X}$ for the unique element in $A_{P}$ such that $H_{P}\left(e^{X}\right)=X$, and for $\lambda \in \mathfrak{A}_{P}^{*}$, we write $e^{\lambda}$ for the character $p \rightarrow e^{\left\langle\lambda, H_{P}(p)\right\rangle}$ of $P(\mathbb{A})$.

As usual, we denote by $\rho_{P} \in \mathfrak{A}_{P}^{*}$ the unique element such that $e^{2\left\langle\rho_{P}, H(m)\right\rangle}=$ $\left|\operatorname{det} A d_{N}(m)\right|$ where $A d_{N}(m)$ is the adjoint action of $m$ on $\operatorname{Lie}(N)$. For suitable normalizations of Haar measure, we have

$$
\int_{G(\mathbb{A})^{1}} f(x) d x=\int_{N(\mathbb{A})} \int_{A_{P}} \int_{M(\mathbb{A})^{1}} \int_{\mathbf{K}} f(n a m k) e^{-2\left\langle\rho_{P}, H(a)\right\rangle} d n d a d m d k .
$$

5. Truncation operators. We recall the definition of Arthur's truncation operators. Let $T \in \mathfrak{A}_{0}$. Following [A2], we define the truncation of a smooth function $\varphi$ on $P \backslash G(\mathbb{A})$ by the formula

$$
\Lambda^{T, P} \varphi(g)=\sum_{R \subset P}(-1)^{d(R)-d(P)} \sum_{\delta \in R \backslash P} \varphi_{R}(\delta g) \hat{\tau}_{R}^{P}(H(\delta g)-T) .
$$

The sums over $\delta$ are all finite by [A1], Lemma 5.1. Note that $\Lambda^{T, P} \varphi=\Lambda^{T, P} \varphi_{P}$. For $\varphi$ invariant under $G$, Langlands' Lemma immediately yields the inversion formula

$$
\varphi(g)=\sum_{P} \sum_{\delta \in P \backslash G} \Lambda^{T, P} \varphi(g) \tau_{P}(H(\delta g)-T) .
$$

From now on, $T \in \mathfrak{A}_{0}$ will denote a sufficiently regular element in the sense of [A2]. In this case, the function $m \longmapsto \Lambda^{T, P} \varphi(m k)$ is rapidly decreasing on $M_{P} \backslash M_{P}(\mathbb{A})^{1}$ for all $k \in \mathbf{K}$.

\section{Periods Relative to Quadratic extensions}

In this section $E / F$ is a quadratic extension of number fields. We write $\mathbb{A}$ for the adeles of $F$ and $\mathbb{A}_{E}$ for the adeles of $E$. Let $H$ be a split, connected reductive group over $F$ and let $G=H_{/ E}$. Then $H(F)$ is a subgroup of $G(E)$. Let $\mathbf{K}_{F}$ and $\mathbf{K}_{E}$ denote fixed good maximal compact subgroups of $H(\mathbb{A})$ and $G\left(\mathbb{A}_{E}\right)$, respectively. If $P=M N$ is a parabolic subgroup of $H$, then

$$
H(\mathbb{A})^{1}=N(\mathbb{A}) A_{P} M(\mathbb{A})^{1} \mathbf{K}_{F},
$$

and a Haar measure on $H(\mathbb{A})^{1}$ is given by the integral

$$
\int_{N(\mathbb{A})} \int_{A_{P}} \int_{M(\mathbb{A})^{1}} \int_{\mathbf{K}_{F}} f(n a m k) e^{-2\left\langle\rho_{P}, H(a)\right\rangle} d n d a d m d k .
$$

There is a bijection between the set of parabolic subgroups of $H$ and $G$. If $P$ is a parabolic subgroup of $H$ (standard and defined over $F$ ), then the corresponding parabolic subgroup of $G$ (also standard and defined over $E$ ) is denoted $P_{E}$ and is characterized by $P_{E}(E)=P(E)$. Note that $H_{E}=G$. We identify $\mathfrak{A}_{P}$ and $\mathfrak{A}_{P_{E}}$ and 
their dual spaces. In this identification the roots of $H$ correspond to the roots of $G$ and hence $\rho_{P}=\rho_{P_{E}}$. Similarly, the standard characteristic functions do not depend on whether they are defined relative to $H$ or $G$, i.e., $\tau_{Q}^{P}=\tau_{Q_{E}}^{P_{E}}, \hat{\tau}_{Q}^{P}=\hat{\tau}_{Q_{E}}^{P_{E}}$, etc. We have

$$
H_{P_{E}}(a)=2 H_{P}(a)
$$

for $a \in H(\mathbb{A})$. We also write $H_{E}$ for the height function on $G$.

For $\varphi \in \mathcal{A}(G)$, we write

$$
\varphi_{P_{E}}(g)=\int_{N(E) \backslash N\left(\mathbb{A}_{E}\right)} \varphi(n g) d n
$$

for the constant term of $\varphi$ relative to the parabolic subgroup $P_{E}$ in $G$. We also fix Siegel domains for $G$ and for the parabolic subgroups $P_{E}$ of $G$. Thus we choose a compact subset $\Theta \subset N_{0}\left(\mathbb{A}_{E}\right) M_{0}\left(\mathbb{A}_{E}\right)^{1}$ and $T_{0} \in \mathfrak{A}_{0}$ such that $\left\langle\alpha, T_{0}\right\rangle<<0$ for all $\alpha \in \Delta_{0}$, and we let $\mathcal{S}^{P_{E}}$ be the set of elements pak where $p \in \Theta, k \in \mathbf{K}_{E}$, and $a \in A_{P_{0}}$ satisfies

$$
\left\langle\alpha, H_{E}(a)\right\rangle \geq\left\langle\alpha, T_{0}\right\rangle \quad \text { for all } \quad \alpha \in \Delta_{0}^{P} .
$$

We write $\mathcal{S}$ for $\mathcal{S}^{G}$. Let $\mathcal{S}^{H}$ be the Siegel domain for $H$ consisting of elements pak such that $p \in \Theta \cap H(\mathbb{A}), k \in \mathbf{K}_{F}$, and $a \in A_{P_{0}}$. By a basic result of reduction theory, we may (and shall) choose $\Theta$ and $T_{0}$ such that $G\left(\mathbb{A}_{E}\right)=P_{E}(E) \mathcal{S}^{P_{E}}$. We may also assume that $H(\mathbb{A})=H(F) \mathcal{S}^{H}$.

6. Mixed truncation. For $T \in \mathfrak{A}_{0}$, we define the mixed truncation of $\varphi$ by the formula

$$
\Lambda_{m}^{T, P} \varphi(h)=\sum_{R \subset P}(-1)^{d(R)-d(P)} \sum_{\delta \in R \backslash P} \varphi_{R_{E}}(\delta h) \hat{\tau}_{R}^{P}\left(H_{E}(\delta h)-T\right) .
$$

This is halfway between truncation on $G$ (since we use constant terms relative to $G$ ) and truncation on $H$ (since the sums only involve $\delta$ lying in $H$ ). We write $\Lambda_{m}^{T}$ for $\Lambda_{m}^{T, H}$. Thus,

$$
\Lambda_{m}^{T} \varphi(h)=\sum_{R \subset H}(-1)^{d(R)-d(G)} \sum_{\delta \in R \backslash H} \varphi_{R_{E}}(\delta h) \hat{\tau}_{R}\left(H_{E}(\delta h)-T\right) .
$$

The following inversion formula holds, as before, for all $h \in H(\mathbb{A})$ :

$$
\varphi(h)=\sum_{P \subset H} \sum_{\delta \in P \backslash H} \Lambda_{m}^{T, P} \varphi(\delta h) \tau_{P}\left(H_{E}(\delta h)-T\right) .
$$

Proposition 8. Assume that $T$ is sufficiently regular. Then for fixed $h$, the function $m \longrightarrow \Lambda_{m}^{T, P} \varphi(m h)$ is rapidly decreasing on $M \backslash M(\mathbb{A})^{1}$.

Proof. We need only check that each step of the argument in [A2], Section 1, applies. It suffices to treat the case $M=H$. If $P_{1} \subset P_{2}$ are parabolic subgroups, set

$$
\phi_{12}(h)=\sum_{\substack{P \\ P_{1} \subset P \subset P_{2}}}(-1)^{d(P)-d(G)} \phi_{P_{E}}(h) .
$$

As in [A2],

$$
\Lambda_{m}^{T, H} \varphi(h)=\sum_{P_{1} \subset P_{2}} \sum_{\delta \in P_{1} \backslash H} F_{H}^{1}\left(\delta h, \frac{1}{2} T\right) \sigma_{1}^{2}\left(H_{E}(\delta h)-T\right) \phi_{12}(\delta h)
$$


(the definitions of the characteristic functions $F^{1}$ and $\sigma_{1}^{2}$ are in $[\mathrm{A} 1] ; F_{H}^{1}$ denotes that $F^{1}$ is taken with respect to $H$ ). The argument of [A2], pp. 96-97, applied to the group $H$ yields the bound

$$
\sum_{\delta \in P_{1} \backslash H} F_{H}^{1}\left(\delta h, \frac{1}{2} T\right) \sigma_{1}^{2}\left(H_{E}(\delta h)-T\right) \leq C_{1}\|h\|^{N_{1}}
$$

for all $h \in H(\mathbb{A})$, for some constants $C_{1}, N_{1}>0$. On the other hand, the argument of [A2], pp. 92-96, for the group $G$ shows that for any $N>0$ there exists $C>0$ such that

$$
\left|\phi_{12}(\delta g)\right| \leq C\|g\|^{-N}
$$

for all $\delta \in G(E)$ and $g \in G(\mathbb{A})^{1}$ with $F_{G}^{1}(\delta g, T) \sigma_{1}^{2}\left(H_{E}(\delta g)-T\right)=1$. However, it is clear from the definition that if $F_{H}^{1}\left(\delta h, \frac{1}{2} T\right)=1$, then $F_{G}^{1}(\delta h, T)=1$. It follows that $\Lambda_{m}^{T, H} \varphi(h)$ is rapidly decreasing on $H(\mathbb{A})^{1}$.

7. The period of an automorphic form. We now define the regularized period of an automorphic form. The first step is to define a certain integral over $P \backslash H(\mathbb{A})^{1}$ where $P$ is a parabolic subgroup of $H$. Let $\tau_{k}(X)$ be a function of type (C) (as defined in Section II) on $\mathfrak{A}_{P}$ that depends continuously on $k \in \mathbf{K}_{E}$, i.e., we assume that there is a decomposition $\mathfrak{A}_{P}=W_{1} \oplus W_{2}$ such that $\tau_{k}$ has the form

$$
g_{k}\left(w_{1}-T_{1}\right) \tau_{\mathcal{C}_{2 k}}\left(w_{2}-T_{2}\right)
$$

where the compactly supported function $g_{k}$ varies continuously in the $L^{1}$-norm and linear inequalities defining the cone $\mathcal{C}_{2 k}$ vary continuously. Let $f$ be a function on $P_{E}(E) N_{E}\left(\mathbb{A}_{E}\right) \backslash G\left(\mathbb{A}_{E}\right)$ of the form

$$
f(n a m k)=\sum_{j=1}^{k} \phi_{j}(m, k) \alpha_{j}\left(H_{P_{E}}(a), k\right) e^{\left\langle\lambda_{j}+\rho_{P_{E}}, H_{E}(a)\right\rangle}
$$

for $n \in N(\mathbb{A}), a \in A_{P}, m \in M(\mathbb{A})^{1}$ and $k \in \mathbf{K}_{E}$, where for all $j$,

(a) $\lambda_{j} \in \mathfrak{A}_{P}^{*}$ and $\alpha_{j}(X, k)$ is a continuous family of polynomials on $\mathfrak{A}_{P}$ such that for all $k \in \mathbf{K}_{E}, \alpha_{j}(X, k) e^{\left\langle\lambda_{j}, X\right\rangle} \tau_{k}(X)$ is \#-integrable;

(b) $\phi_{j}(m, k)$ is absolutely integrable on $M \backslash M(\mathbb{A})^{1} \times \mathbf{K}_{F}$.

In this case, we define the \#-integral

$$
\int_{P \backslash H(\mathbb{A})^{1}}^{\#} f(h) \tau_{k}\left(H_{P_{E}}(h)\right) d h
$$

by

$$
\sum_{j=1}^{k} \int_{\mathbf{K}_{F}}\left(\int_{M \backslash M(\mathbb{A})^{1}} \phi_{j}(m k) d m\right)\left(\int_{\mathfrak{A}_{P}}^{\#} \alpha_{j}(2 X, k) e^{\left\langle\lambda_{j}, 2 X\right\rangle} \tau_{k}(2 X) d X\right) d k
$$

Recall that $H_{P_{E}}(a)=2 H_{P}(a)$ for $a \in A_{P}$ and therefore, with our definition of the exponents $\lambda_{j}$, no shift by $\rho_{P}$ appears in the \#-integral over $\mathfrak{A}_{P}$.

We write $\mathcal{E}_{P}(f)$ for the set of distinct exponents $\left\{\lambda_{j}\right\}$ occurring in (20). This set is uniquely determined by $f$, but the functions $\alpha_{j}$ and $\phi_{j}$ are not. However, if we denote by $e^{X}$ the element in $A_{P}$ such that $H_{P}\left(e^{X}\right)=X$, then the function

$$
X \longrightarrow \int_{M \backslash M(\mathbb{A})^{1}} f\left(e^{X} m k\right) d m
$$


is an exponential polynomial on $\mathfrak{A}_{P}$ and (21) is equal to

$$
\int_{\mathbf{K}_{F}} \int_{\mathfrak{A}_{P}}^{\#}\left(\int_{M \backslash M(\mathbb{A})^{1}} f\left(e^{X} m k\right) d m\right) e^{-2\left\langle\rho_{P}, X\right\rangle} \tau_{k}(2 X) d X d k .
$$

This shows, in particular, that (21) is independent of the decomposition (20). If each of the exponents $\lambda_{j}$ are negative with respect to $\mathcal{C}_{2 k}$ for all $k \in \mathbf{K}_{F}$, then the ordinary integral

$$
\int_{P \backslash H(\mathbb{A})^{1}} f(h) \tau_{k}\left(H_{P_{E}}(h)\right) d h
$$

is absolutely convergent and its value coincides with the \#-integral by Lemma 2 .

We fix a sufficiently regular element $T \in \mathfrak{A}_{0}^{+}$. Then the above construction applies to $\Lambda_{m}^{T, P} \Psi(g)$ and the characteristic function $\tau_{P}\left(H_{E}(g)-T\right)$ where $\Psi \in$ $\mathcal{A}_{P_{E}}(G)$. According to [MW], I.3.2, $\Psi$ has a decomposition of the type (20). Namely,

$$
\Psi(n a m k)=\sum_{j=1}^{r} Q_{j}\left(H_{P_{E}}(a)\right) \psi_{j}(a m k)
$$

for $n \in N\left(\mathbb{A}_{E}\right), a \in A_{P_{E}}, m \in M\left(\mathbb{A}_{E}\right)^{1}$ and $k \in \mathbf{K}_{E}$, where the $Q_{j}$ are polynomials and $\psi_{j} \in \mathcal{A}_{P_{E}}(G)$ satisfies

$$
\psi_{j}(a g)=e^{\left\langle\lambda_{j}+\rho_{P}, H_{E}(a)\right\rangle} \psi_{j}(g)
$$

for some exponent $\lambda_{j} \in \mathfrak{A}_{P}^{*}$ for all $a \in A_{P_{E}}$. By Proposition 8, the functions $m \longrightarrow \Lambda_{m}^{T, P} \psi_{j}(m k)$ are rapidly decreasing and hence absolutely integrable over $M \backslash M(\mathbb{A})^{1} \times \mathbf{K}_{F}$. Since $\tau_{P}$ is the characteristic function of the cone spanned by the coweights $\hat{\Delta}_{P}^{\vee}$ we see that

$$
\int_{P \backslash H(\mathbb{A})^{1}}^{\#} \Lambda_{m}^{T, P} \Psi(h) \tau_{P}\left(H_{P_{E}}(h)-T\right) d h
$$

exists if and only if

$$
\left\langle\lambda_{j}, \varpi^{\vee}\right\rangle \neq 0 \text { for all } \varpi^{\vee} \in \hat{\Delta}_{P}^{\vee} \text { and } \lambda_{j} \in \mathcal{E}_{P}(\Psi) .
$$

The same is true for $\Lambda^{T, P} \Psi(h) \tau_{P}\left(H_{P_{E}}(h x)-T\right)$ for fixed $x \in H(\mathbb{A})$. Indeed, for $h \in H(\mathbb{A})$, let $K(h) \in \mathbf{K}_{F}$ be any element such that $h K(h)^{-1} \in P_{0}(\mathbb{A})$. Then

$$
H_{P_{E}}(h x)=H_{P_{E}}(h)+H_{P_{E}}(K(h) x)
$$

and hence $\tau_{P}\left(X-T+H_{P_{E}}(K(h) x)\right)$ is the characteristic function of a cone depending continuously on $h$. We set

$$
\Pi_{P}^{H, T}(\Psi)=\int_{P \backslash H(\mathbb{A})^{1}}^{\#} \Lambda_{m}^{T, P} \Psi(h) \tau_{P}\left(H_{P_{E}}(h)-T\right) d h .
$$

For any automorphic form $\varphi \in \mathcal{A}(G)$, we write $\mathcal{E}_{P}(\varphi)$ for the set of exponents $\mathcal{E}_{P}\left(\varphi_{P}\right)$. Set

$$
\mathcal{A}(G)^{*}=\left\{\varphi \in \mathcal{A}(G):\left\langle\lambda, \varpi^{\vee}\right\rangle \neq 0 \text { for all } \varpi^{\vee} \in \hat{\Delta}_{P}^{\vee}, \lambda \in \mathcal{E}_{P}(\varphi), P \neq H\right\} .
$$

If $\varphi \in \mathcal{A}(G)^{*}$, then $\Pi_{P}^{H, T}\left(\varphi_{P}\right)$ exists for all $P$, and we can define the regularized period

$$
\int_{H \backslash H(\mathbb{A})^{1}}^{*} \varphi(h) d h=\sum_{P \subset H} \Pi_{P}^{H, T}\left(\varphi_{P}\right) .
$$


We also denote this integral by $\Pi^{G / H}(\varphi)$. The name and notations are justified by Theorem 9 below.

Let $\mathbb{A}_{E f}$ be the finite adeles of $\mathbb{A}_{E}$. For $x \in G\left(\mathbb{A}_{E f}\right)$, let $\rho(x)$ denote right translation by $x: \rho(x) \varphi(g)=\varphi(g x)$. The space $\mathcal{A}(G)$ is stable under right translation by $G\left(\mathbb{A}_{f}\right)$. Furthermore, $\rho(x) \varphi$ has the same set of exponents as $\varphi$. Indeed, for $k \in \mathbf{K}_{E}$, write the Iwasawa decomposition of $k x$ as $k x=n^{\prime} a^{\prime} m^{\prime} K(k x)$ and write $\varphi_{P_{E}}$ in the form $(22)$ :

$$
\varphi_{P_{E}}(n a m k)=\sum_{j=1}^{r} Q_{j}\left(H_{P_{E}}(a)\right) e^{\left\langle\lambda_{j}+\rho_{P_{E}}, H_{E}(a)\right\rangle} \psi_{j}(m k) .
$$

Since $a m k x=n^{\prime \prime} a a^{\prime} m m^{\prime} K(k x)$ for some $n^{\prime \prime} \in N_{E}\left(\mathbb{A}_{E}\right), \varphi_{P_{E}}(n a m k x)$ is equal to

$$
\sum_{j=1}^{r} Q_{j}\left(H_{P_{E}}(a)+H_{P_{E}}\left(a^{\prime}\right)\right) e^{\left\langle\lambda_{j}+\rho_{P_{E}}, H_{E}(a)+H_{E}\left(a^{\prime}\right)\right\rangle} \psi_{j}\left(m m^{\prime} K(k x)\right)
$$

and this shows that $\mathcal{E}_{P}(\rho(x) \varphi)=\mathcal{E}_{P}(\varphi)$ as claimed. It follows that the space $\mathcal{A}(G)^{*}$ is invariant under right translation by $G\left(\mathbb{A}_{E f}\right)$.

Theorem 9. (i) $\Pi^{G / H}$ defines an $H\left(\mathbb{A}_{f}\right)^{1}$-invariant linear functional on $\mathcal{A}(G)^{*}$.

(ii) $\Pi^{G / H}$ is independent of the choice of $T$.

(iii) If $\varphi \in \mathcal{A}(G)$ is integrable over $H \backslash H(\mathbb{A})^{1}$, then $\varphi \in \mathcal{A}(G)^{*}$ and

$$
\Pi^{G / H}(\varphi)=\int_{H \backslash H(\mathbb{A})^{1}} \varphi(h) d h .
$$

Proof. We first observe that for $f$ defined by (20), we have

$$
\int_{P \backslash H(\mathbb{A})^{1}}^{\#} f(h x) \tau_{k}(h x) d g=\int_{P \backslash H(\mathbb{A})^{1}}^{\#} f(h) \tau_{k}(h) d h
$$

for all $x \in H\left(\mathbb{A}_{f}\right)^{1}$. To verify this, set $f_{\mu}(g)=e^{\left(\mu, H_{P_{E}}(g)\right)} f(g)$ for $\mu \in \mathfrak{A}_{P}^{*}$. If $\left\langle\operatorname{Re} \mu, \varpi^{\vee}\right\rangle<<0$ for all $\varpi^{\vee} \in \hat{\Delta}_{P}^{\vee}$, then

$$
\int_{P \backslash H(\mathbb{A})^{1}} f_{\mu}(h x) \tau_{k}(h x) d h=\int_{P \backslash H(\mathbb{A})^{1}} f_{\mu}(h) \tau_{k}(h) d h,
$$

by the invariance of Haar measure, since both sides are absolutely convergent. Both sides have a meromorphic continuation whose value at $\mu=0$ gives (24).

Now fix $x \in H\left(\mathbb{A}_{f}\right)^{1}$ and set

$$
F_{P}(g)=\left(\Lambda_{m}^{T, P} \rho\left(x^{-1}\right) \varphi\right)(g x) .
$$

Then

$$
\begin{array}{r}
\int_{P \backslash H(\mathbb{A})^{1}}^{\#}\left(\Lambda_{m}^{T, P} \rho\left(x^{-1}\right) \varphi\right)(h) \tau_{P}\left(H_{P_{E}}(h)-T\right) d h \\
=\int_{P \backslash H(\mathbb{A})^{1}}^{\#} F_{P}(h) \tau_{P}\left(H_{P_{E}}(h x)-T\right) d h
\end{array}
$$

by (24), and hence we must show that

$$
\int_{H \backslash H(\mathbb{A})^{1}}^{*} \varphi(h) d h=\sum_{P} \int_{P \backslash H(\mathbb{A})^{1}}^{\#} F_{P}(h) \tau_{P}\left(H_{P_{E}}(h x)-T\right) d h .
$$


Let us re-write $F_{P}$ using the functions $\Gamma_{Q}^{P}$ of $\S 3$. As before, for $h \in H(\mathbb{A})$, we let $K(h) \in \mathbf{K}$ be any element such that $h K(h)^{-1} \in P_{0}(\mathbb{A})$. We claim that

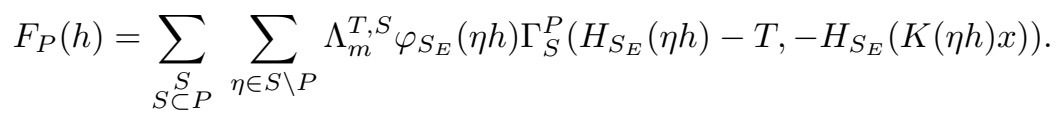

Indeed, by definition,

$$
F_{P}(h)=\sum_{\substack{R \\ R \subset P}}(-1)^{d(R)-d(P)} \sum_{\delta \in R \backslash P} \varphi_{R_{E}}(\delta h) \hat{\tau}_{R}^{P}\left(H_{E}(\delta h x)-T\right)
$$

and

$$
H_{E}(\delta h x)-T=H_{E}(\delta h)-T+H_{E}(K(\delta h) x) .
$$

Using (17), we may write $F_{P}(h)$ as the double sum over all $R \subset S \subset P$ and $\delta \in R \backslash P$ of

$$
(-1)^{d(R)-d(S)} \varphi_{R_{E}}(\delta h) \hat{\tau}_{R}^{S}\left(H_{E}(\delta h)-T\right) \Gamma_{S}^{P}\left(H_{S_{E}}(\delta h)-T,-H_{S_{E}}(K(\delta h) x)\right) .
$$

This equals the sum over $S \subset P$ and $\eta \in S \backslash P$ of

$$
\begin{aligned}
\left(\sum_{R \subset S}(-1)^{d(R)-d(S)} \sum_{\delta \in R \backslash S} \varphi_{R_{E}}(\delta \eta h) \hat{\tau}_{R}^{S}\left(H_{E}(\delta \eta h)-T\right)\right) \\
\times \Gamma_{S}^{P}\left(H_{S_{E}}(\eta h)-T,-H_{S_{E}}(K(\eta h) x)\right)
\end{aligned}
$$

and (26) follows.

This gives

$$
\begin{aligned}
& \int_{P \backslash H(\mathbb{A})^{1}}^{\#} F_{P}(h) \tau_{P}\left(H_{P_{E}}(h x)-T\right) d h \\
&= \int_{P \backslash H(\mathbb{A})^{1}}^{\#} \sum_{S \subseteq P} \sum_{\substack{S \\
\subset \in S \backslash P}} \Lambda_{m}^{T, S} \varphi_{S_{E}}(\eta h) \Gamma_{S}^{P}\left(H_{S_{E}}(\eta h)-T,-H_{S_{E}}(K(\eta h) x)\right) \\
& \times \tau_{P}\left(H_{P_{E}}(h x)-T\right) d h
\end{aligned}
$$

and our next step is to show that the sum over $S$ can be taken outside of the integral. Recall that the functions $\Gamma_{S}^{P}(Z, W)$ depend only on the projections of $Z$ and $W$ onto $\mathfrak{A}_{S}^{P}$. According to [A3], Lemma 2.1, for $W$ belonging to a fixed compact subset of $\mathfrak{A}_{S}^{P}$, there exists a compact subset $\mathcal{Y} \subset \mathfrak{A}_{S}^{P}$ such that the function $Z \longrightarrow \Gamma_{S}^{P}(Z, W)$ is the characteristic function of a compact set in $\mathfrak{A}_{S}^{P}$ contained in $\mathcal{Y}$. In particular, the function

$$
h \longrightarrow \Gamma_{S}^{P}\left(H_{S_{E}}(h)-T,-H_{S_{E}}(K(h) x)\right)
$$

is supported inside a subset of elements $h$ for which the projection of $H_{S_{E}}(h)$ onto $\mathfrak{A}_{S}^{P}$ lies in a compact set depending only on $x$. Therefore

$$
\sum_{\eta \in S \backslash P} \Lambda^{T, S} \varphi_{S_{E}}(\eta h) \Gamma_{S}^{P}\left(H_{S_{E}}(\eta h)-T,-H_{S_{E}}(K(\eta h) x)\right)
$$

is integrable over $M(F) \backslash M(\mathbb{A})^{1}$. Now let $h=n e^{X} m k$ be an Iwasawa decomposition of $h$ relative to $P$ with $X \in \mathfrak{A}_{P}$. There exist polynomials $Q_{i}$ on $\mathfrak{A}_{P}$, automorphic 
forms $\psi_{j} \in \mathcal{A}_{P}(G)$, and exponents $\lambda_{j}$ in the $\mathcal{E}_{P}(\varphi)$ such that for all $S \subset P$,

$$
\Lambda_{m}^{T, S} \varphi_{S_{E}}(h)=\sum_{j} Q_{j}(2 X) e^{\left\langle\lambda_{j}+\rho_{P}, 2 X\right\rangle} \Lambda_{m}^{T, S} \psi_{j}(m k) .
$$

Since $\Gamma_{S}^{P}(Z, W)$ depend only on the projections of $Z$ and $W$ onto $\mathfrak{A}_{Q}^{P}$, we may write (27) as the integral over $k \in \mathbf{K}_{F}$ and sum over $j$ of

$$
\begin{aligned}
& \int_{\mathfrak{A}_{P}}^{\#} \int_{M \backslash M(\mathbb{A})^{1}} \sum_{S \subset P} \sum_{\eta \in S \backslash P} Q_{j}(2 X) e^{\left\langle\lambda_{j}, 2 X\right\rangle} \Lambda_{m}^{T, S} \psi_{j}(\eta m k) \\
& \times \Gamma_{S}^{P}\left(H_{S_{E}}(\eta m)-T,-H_{S_{E}}(K(\eta m k) x)\right) d m \tau_{P}\left(2 X+H_{E}(k x)-T\right) d X \\
& =\int_{\mathfrak{A}_{P}}^{\#} \sum_{j} Q_{j}(2 X) e^{\left\langle\lambda_{j}, 2 X\right\rangle} \tau_{P}\left(2 X+H_{E}(k x)-T\right) d X \int_{M \backslash M(\mathbb{A})^{1}} \sum_{S \subseteq P} \sum_{\eta \in S \backslash P} \sum_{j} \\
& \times \Lambda_{m}^{T, S} \psi_{j}(\eta m k) \Gamma_{S}^{P}\left(H_{S_{E}}(\eta m)-T,-H_{S_{E}}(K(\eta m k) x)\right) d m
\end{aligned}
$$

and since each term in the sum over $S$ is separately integrable over $M \backslash M(\mathbb{A})^{1}$, we may take the sum over $S$ outside the integral as claimed.

We now claim that

$\int_{P \backslash H(\mathbb{A})^{1}}^{\#} \sum_{\eta \in S \backslash P} \Lambda^{T, S} \varphi_{S_{E}}(\eta h) \Gamma_{S}^{P}\left(H_{S_{E}}(\eta h)-T,-H_{S_{E}}(K(\eta h) x)\right) \tau_{P}\left(H_{E}(h x)-T\right) d h$ is equal to

$$
\int_{S \backslash H(\mathbb{A})^{1}}^{\#} \Lambda^{T, S} \varphi_{S_{E}}(h) \Gamma_{S}^{P}\left(H_{S_{E}}(h)-T,-H_{S_{E}}(K(h) x)\right) \tau_{P}\left(H_{E}(h x)-T\right) d h .
$$

Indeed, (29) is equal to the integral over $k \in \mathbf{K}_{F}$ and sum over $j$ of

$$
\begin{aligned}
& \int_{\mathfrak{A}_{P}}^{\#} Q_{j}(2 X) e^{\left\langle\lambda_{j}, 2 X\right\rangle} \tau_{P}\left(2 X+H_{E}(k x)-T\right) d X \\
& \quad \times \int_{M \backslash M(\mathbb{A})^{1}} \sum_{\eta \in S \backslash P} \Lambda_{m}^{T, S} \psi_{j}(\eta m k) \Gamma_{S}^{P}\left(H_{S_{E}}(\eta m)-T,-H_{S_{E}}(K(\eta m k) x)\right) d m
\end{aligned}
$$

which can be written

$$
\begin{aligned}
\int_{\mathfrak{A}_{P}}^{\#} & Q_{j}(2 X) e^{\left\langle\lambda_{j}, X\right\rangle} \tau_{P}\left(2 X+H_{E}(k x)-T\right) d X \\
& \times \int_{S_{M} \backslash M(\mathbb{A})^{1}} \Lambda_{m}^{T, S} \psi_{j}(m k) \Gamma_{S}^{P}\left(H_{S_{E}}(m)-T,-H_{S_{E}}(K(m k) x)\right) d m
\end{aligned}
$$

where $S_{M}=S \cap M$. Expressing the integral over $M_{S} \backslash M(\mathbb{A})^{1}$ using the Iwasawa decomposition $m=n^{\prime} e^{X^{\prime}} m^{\prime} k^{\prime}$ of $M(\mathbb{A})^{1}$ relative to $S_{M}$ gives

$$
\begin{aligned}
& \int_{\mathfrak{A}_{P}}^{\#} Q_{j}(2 X) e^{\left\langle\lambda_{j}, 2 X\right\rangle} \tau_{P}\left(2 X+H_{E}(k x)-T\right) d X \int_{\mathbf{K}_{M}} \int_{\mathfrak{A}_{S}^{P}}^{\#} \int_{M_{S} \backslash M_{S}(\mathbb{A})^{1}} \\
& \times e^{-2\left\langle\rho_{S}^{P}, X^{\prime}\right\rangle} \Lambda_{m}^{T, S} \psi_{j}\left(e^{X^{\prime}} m^{\prime} k^{\prime} k\right) \Gamma_{S}^{P}\left(2 X^{\prime}-T,-H_{S_{E}}\left(k^{\prime} k x\right)\right) d m^{\prime} d X^{\prime} d k^{\prime}
\end{aligned}
$$

where $\mathbf{K}_{M}=\mathbf{K}_{F} \cap M(\mathbb{A})^{1}$. Since we are integrating over $\mathbf{K}_{F}$, we may drop the integral over $\mathbf{K}_{M}$. However, each function $\Lambda_{m}^{T, S} \psi_{j}\left(e^{X^{\prime}} m^{\prime} k\right)$ has a decomposition 
analogous to (28) with respect to $S$ and therefore, for fixed $k$, the function

$$
\left(m^{\prime}, X^{\prime}\right) \longrightarrow \sum_{j} \Lambda_{m}^{T, S} \psi_{j}\left(e^{X^{\prime}} m^{\prime} k\right) \Gamma_{S}^{P}\left(2 X^{\prime}-T,-H_{S_{E}}(k x)\right) d m^{\prime}
$$

is a sum of terms, each of which is the product of a function of $m^{\prime}$ which is absolutely integrable over $M_{S} \backslash M_{S}(\mathbb{A})^{1}$ and a function of $X^{\prime}$ which itself is equal to an exponential polynomial times the compactly supported function $\Gamma_{S}^{P}\left(2 X^{\prime}-T,-H_{S_{E}}(k x)\right)$. According to (14), we may combine the integrals over $\mathfrak{A}_{S}^{P}$ and $\mathfrak{A}_{P}$ to a \#-integral over $\mathfrak{A}_{S}$, and we find that (29) is equal to the integral over $k \in \mathbf{K}_{F}$ and $m^{\prime} \in M_{S} \backslash M_{S}(\mathbb{A})^{1}$ of

$\int_{\mathfrak{A}_{S}}^{\#} e^{-2\left\langle\rho_{P}, X\right\rangle} \Lambda_{m}^{T, S} \varphi_{S_{E}}\left(e^{X} m^{\prime} k\right) \Gamma_{S}^{P}\left(2 X-T,-H_{S_{E}}(k x)\right) \tau_{P}\left(2 X+H_{E}(k x)-T\right) d X$, and this is equal to (30).

Summing (30) over all $S$ and $P$ such that $S \subset P$, we see that

$$
\sum_{P} \int_{P \backslash H(\mathbb{A})^{1}}^{\#} F_{P}(h) d h
$$

is equal to the sum over parabolic subgroups $S$ of

$$
\sum_{P \supset} \int_{S \backslash H(\mathbb{A})^{1}}^{\#} \Lambda_{m}^{T, S} \varphi_{S_{E}}(h) \Gamma_{S}^{P}\left(H_{S_{E}}(h)-T,-H_{S_{E}}(K(h) x)\right) \tau_{P}\left(H_{E}(h x)-T\right) d h
$$

and (25) will follow if we prove that (31) is equal to

$$
\int_{S \backslash H(\mathbb{A})^{1}}^{\#} \Lambda^{T, S} \varphi_{S_{E}}(h) \tau_{S}\left(H_{E}(h)-T\right) d h .
$$

The relation (18):

$$
\tau_{S}(Y-X)=\sum_{P \supset S} \Gamma_{S}^{P}(Y-X,-X) \tau_{P}(Y)
$$

applied to $Y=H_{S_{E}}(h x)-T$ and $X=H_{S_{E}}(h x)-H_{S_{E}}(h)=H_{S_{E}}(K(h) x)$ gives

$$
\sum_{P \supset S} \Gamma_{S}^{P}\left(H_{S_{E}}(h)-T,-H_{S_{E}}(K(h) x)\right) \tau_{P}\left(H_{E}(h x)-T\right)=\tau_{S}\left(H_{E}(h)-T\right) .
$$

Thus we need to show that the summation over $P$ in (31) can be taken inside the \#-integral.

Let $h=n e^{X} m k$ with $X \in \mathfrak{A}_{S}$ be the Iwasawa decomposition of $h \in H(\mathbb{A})$ relative to $S$. Then

$$
\Gamma_{S}^{P}\left(H_{S_{E}}(h)-T,-H_{S_{E}}(K(h) x)\right) \tau_{P}\left(H_{E}(h x)-T\right)
$$

is equal to

$$
\Gamma_{S}^{P}\left(2 X-T,-H_{S_{E}}(k x)\right) \tau_{P}\left(2 X+H_{E}(k x)-T\right) .
$$

Since the subset $\left\{H_{S_{E}}(k x): k \in H(\mathbb{A})\right\}$ of $\mathfrak{A}_{S}$ is compact, Lemma 2.1 of [A3] cited above implies that the function $Z \longrightarrow \Gamma_{S}^{P}\left(Z,-H_{S_{E}}(k x)\right)$ is supported in a fixed compact set independent of $k$. The cone defining $\tau_{P}$ is the positive Weyl chamber in $\mathfrak{A}_{P}$ which is contained in the positive Weyl chamber of $\mathfrak{A}_{S}$. Thus, we may apply 
Lemma 6 to take the sum over $P$ inside the integral. This completes the proof of (i).

The proof of (ii) is nearly identical. Suppose that $T^{\prime} \in \mathfrak{A}_{0}$ is regular. We have

$$
\Lambda_{m}^{T+T^{\prime}, P} \varphi(h)=\sum_{\substack{Q \\ Q \subset P}} \sum_{\delta \in Q \backslash P} \Lambda_{m}^{T, Q} \varphi(\delta h) \Gamma_{Q}^{P}\left(H_{E}(\delta h)-T, T^{\prime}\right) .
$$

Indeed, using the formula

$$
\hat{\tau}_{R}^{P}\left(H(\delta h)-T-T^{\prime}\right)=\sum_{\substack{Q \\ R \subset Q \subset P}}(-1)^{d(Q)-d(P)} \hat{\tau}_{R}^{Q}\left(H_{E}(\delta h)-T\right) \Gamma_{Q}^{P}\left(H_{E}(\delta h)-T, T^{\prime}\right),
$$

we have

$$
\begin{aligned}
\Lambda_{m}^{T+T^{\prime}, P} \varphi(h)=\sum_{\substack{R \\
R \subset P}}(-1)^{d(R)-d(P)} \sum_{\delta \in R \backslash P} \varphi_{R_{E}}(\delta h) \hat{\tau}_{R}^{P}\left(H_{E}(\delta h)-T-T^{\prime}\right) \\
=\sum_{\substack{R, Q \\
R \subset Q \subset P}} \sum_{\delta \in Q \backslash P}(-1)^{d(R)-d(Q)} \sum_{\gamma \in R \backslash Q} \varphi_{R_{E}}(\delta h) \hat{\tau}_{R}^{Q}\left(H_{E}(\gamma \delta h)-T\right) \Gamma_{Q}^{P}\left(H_{E}(\delta h)-T, T^{\prime}\right) \\
=\sum_{\substack{Q \\
Q \subset P}} \sum_{\delta \in Q \backslash P} \Lambda_{m}^{T, Q} \varphi(\delta h) \Gamma_{Q}^{P}\left(H_{E}(\delta h)-T, T^{\prime}\right) .
\end{aligned}
$$

Therefore

$$
\begin{aligned}
& \sum_{P} \int_{P \backslash H(\mathbb{A})^{1}}^{\#} \Lambda_{m}^{T+T^{\prime}, P} \varphi(h) \tau_{P}\left(H_{E}(h)-T-T^{\prime}\right) d h \\
& =\sum_{P} \int_{P \backslash H(\mathbb{A})^{1}}^{\#} \sum_{\substack{Q \\
Q \subset P}} \sum_{\delta \in Q \backslash P} \Lambda_{m}^{T, Q} \varphi(\delta h) \Gamma_{Q}^{P}\left(H_{E}(\delta h)-T, T^{\prime}\right) \tau_{P}\left(H_{E}(h)-T-T^{\prime}\right) d h \\
& =\sum_{Q, P} \int_{Q \backslash H(\mathbb{A})^{1}}^{\#} \Lambda_{m}^{T, Q} \varphi(h) \Gamma_{Q}^{P}\left(H_{E}(h)-T, T^{\prime}\right) \tau_{P}\left(H_{E}(h)-T-T^{\prime}\right) d h \\
& =\sum_{Q} \int_{Q \backslash H(\mathbb{A})^{1}}^{\#} \Lambda_{m}^{T, Q} \varphi(g) \sum_{P} \Gamma_{Q}^{P}\left(H_{E}(h)-T, T^{\prime}\right) \tau_{P}\left(H_{E}(h)-T-T^{\prime}\right) d h
\end{aligned}
$$

where the second equality is justified in the same way as the equality of (29) and (30) and the third equality is justified as in the discussion of (31) above. The relation

$$
\sum_{P \supset}^{P} \Gamma_{Q}^{P}\left(H_{E}(g)-T, T^{\prime}\right) \tau_{P}\left(H_{E}(g)-T-T^{\prime}\right)=\tau_{Q}\left(H_{E}(g)-T\right)
$$

follows from (18), and we obtain

$$
\sum_{Q} \int_{Q \backslash H(\mathbb{A})^{1}}^{\#} \Lambda_{m}^{T, Q} \varphi(g) \tau_{Q}(H(g)-T) d g=\int_{H \backslash H(\mathbb{A})}^{*} \varphi(g) d g
$$

as required. 
We now prove (iii). By (19), it will suffice to check that if $\varphi \in \mathcal{A}(G)^{*}$ is integrable over $H \backslash H(\mathbb{A})^{1}$, then the integral

$$
\int_{P \backslash H(\mathbb{A})^{1}} \Lambda_{m}^{T, P} \varphi_{P_{E}}(h) \tau_{P}\left(H_{E}(h)-T\right) d h
$$

converges absolutely and is equal to $\Pi_{P}^{H, T}\left(\varphi_{P}\right)$. Expand $\varphi_{P_{E}}$ as in $(22)$. As shown in [MW], top of p. 50, for all $j$, there exists a parabolic subgroup $Q \subset P$ and a cuspidal exponent $\mu$ of $\varphi_{Q_{E}}^{\text {cusp }}$ such that $\lambda_{j}$ is equal to the restriction of $\mu$ to $\mathfrak{A}_{P}$ relative to the decomposition

$$
\mathfrak{A}_{Q}=\mathfrak{A}_{Q}^{P} \oplus \mathfrak{A}_{P} .
$$

According to Lemma I.4.11 of [MW], p. 75, if $\varphi$ is square-integrable on $G \backslash G(\mathbb{A})^{1}$, then the exponent $\mu$ can be written in the form $\sum_{\alpha \in \Delta_{P}} x_{\alpha} \alpha$ with $x_{\alpha}<0$. A nearly identical argument shows that this remains true if $\varphi$ is assumed to be integrable over $H \backslash H(\mathbb{A})^{1}$. This says that $\mu$ is negative with respect to the cone $\left\{X \in \mathfrak{A}_{P}: \tau_{P}(X)=1\right\}$. Therefore the integral $\Pi_{P}^{H, T}\left(\varphi_{P}\right)$ is absolutely convergent and coincides with the ordinary integral over $P(F) \backslash H(\mathbb{A})^{1}$ as required.

8. Period of a truncated automorphic form. Let $P=M N$ be a parabolic subgroup and $\varphi \in \mathcal{A}_{P_{E}}(G)$. We may generalize the construction of the previous section to define the regularized integral

$$
\int_{P \backslash H(\mathbb{A})^{1}}^{*} \varphi(h) \tau\left(H_{P_{E}}(h)-T\right) d h
$$

where $\tau$ is a function of type $(\mathrm{C})$ on $\mathfrak{A}_{P}$. Suppose that

$$
\varphi(n a m k)=\sum_{j=1}^{r} Q_{j}\left(H_{E}(a)\right) \psi_{j}(a m k)
$$

as in (22) where $Q_{j}$ and $\psi_{j}$ are as above. Then we set (35) equal to

$$
\sum_{j=1}^{r} \int_{\mathbf{K}_{F}} \Pi^{M_{E} / M}\left(\psi_{j}(\cdot k)\right) d k \int_{\mathfrak{A}_{P}}^{\#} Q_{j}(2 X) e^{\left\langle\lambda_{j}, 2 X\right\rangle} \tau(2 X-T) d X
$$

or

$$
\int_{\mathbf{K}_{F}} \int_{\mathfrak{A}_{P}}^{\#}\left(\int_{M \backslash M(\mathbb{A})^{1}}^{*} \varphi\left(e^{X} m k\right) d m\right) e^{-2\left\langle\rho_{P}, X\right\rangle} \tau(2 X-T) d X d k .
$$

For $\tau=\tau_{P}$ this is well-defined provided that the following two conditions are satisfied:

$\left(1^{*}\right)\left\langle\mu, \varpi^{\vee}\right\rangle \neq 0$ for all $Q \subset P, \varpi^{\vee} \in\left(\hat{\Delta}^{\vee}\right)_{Q}^{P}, \mu \in \mathcal{E}_{Q}(\varphi)$.

$\left(2^{*}\right)\left\langle\lambda, \alpha^{\vee}\right\rangle \neq 0$ for all $\alpha \in \Delta_{P}$, and $\lambda \in \mathcal{E}_{P}(\varphi)$.

Let $\mathcal{A}(G)^{* *}$ be the space of $\varphi \in \mathcal{A}(G)$ such that $\varphi_{P_{E}}$ satisfies $\left(1^{*}\right)$ (and then also $\left.\left(2^{*}\right)\right)$ for all $P$.

Theorem 10. For $\varphi \in \mathcal{A}(G)^{* *}$,

$$
\int_{H \backslash H(\mathbb{A})^{1}} \Lambda_{m}^{T} \varphi(h) d h
$$


is equal to

$$
\sum_{P \subset H}(-1)^{d(P)-d(G)} \int_{P \backslash H(\mathbb{A})^{1}}^{*} \varphi_{P_{E}}(h) \hat{\tau}_{P}\left(H_{E}(h)-T\right) d h .
$$

Proof. By induction on the rank, we may assume that the theorem holds for pairs $\left(M_{E}, M\right)$ where $M$ is the Levi subgroup of a proper parabolic subgroup $P$ of $H$. We will show below that this induction hypothesis implies that

$$
\int_{P \backslash H(\mathbb{A})^{1}}^{\#} \Lambda_{m}^{T, P} \varphi_{P_{E}}(h) \tau_{P}\left(H_{E}(h)-T\right)
$$

is equal to

$$
\sum_{\substack{R \\ R \subset P}}(-1)^{d(R)-d(P)} \int_{R(F) \backslash H(\mathbb{A})^{1}}^{*} \varphi_{R_{E}}(h) \hat{\tau}_{R}^{P}\left(H_{E}(h)-T\right) \tau_{P}\left(H_{E}(h)-T\right) d h .
$$

Assuming this, we may sum over $P$ to write

$$
\int_{H(F) \backslash H(\mathbb{A})^{1}}^{*} \varphi(h) d h-\int_{H(F) \backslash H(\mathbb{A})^{1}} \Lambda^{T} \varphi(h) d h
$$

as

$$
\sum_{\substack{P \neq G \\ P \supset R}}(-1)^{d(R)-d(P)} \int_{R(F) \backslash H(\mathbb{A})^{1}}^{*} \varphi_{R_{E}}(h) \hat{\tau}_{R}^{P}\left(H_{E}(h)-T\right) \tau_{P}\left(H_{E}(h)-T\right) d h .
$$

For $R \neq H$, Langlands' Combinatorial Lemma gives

$$
\begin{aligned}
& \sum_{\substack{P \\
R \subset P \neq H}}(-1)^{d(R)-d(P)} \hat{\tau}_{R}^{P}\left(H_{E}(h)-T\right) \tau_{P}\left(H_{E}(h)-T\right) \\
& \quad=-(-1)^{d(R)-d(H)} \hat{\tau}_{R}\left(H_{E}(h)-T\right)
\end{aligned}
$$

and so the theorem will follow if we check that the summation can be taken inside the integral in (39).

Consider the three cones

$$
\begin{aligned}
& \widehat{\mathcal{C}}_{R}=\left\{X \in \mathfrak{A}_{R}: \hat{\tau}_{R}(X)=1\right\} \\
& \mathcal{C}_{P}=\left\{X \in \mathfrak{A}_{P}: \tau_{P}(X)=1\right\} \\
& \widehat{\mathcal{C}}_{R}^{P}=\left\{X \in \mathfrak{A}_{R}^{P}: \hat{\tau}_{R}^{P}(X)=1\right\} .
\end{aligned}
$$

The product $\mathcal{C}_{P} \times \widehat{\mathcal{C}}_{R}^{P}$ is contained in $\widehat{\mathcal{C}}_{R}$. Indeed, $\widehat{\mathcal{C}}_{R}$ is the positive span of the coroots $\left\{\alpha^{\vee}: \alpha \in \Delta_{R}\right\}, \mathcal{C}_{P}$ is the positive span of the coweights in $\hat{\Delta}_{P}^{\vee}$ and $\widehat{\mathcal{C}}_{R}^{P}$ is the positive span of the coroots $\left\{\alpha^{\vee}: \alpha \in \Delta_{R}^{P}\right\}$, so the assertion follows from the fact that all coweights in $\hat{\Delta}_{P}^{\vee}$ are non-negative linear combinations of coroots in $\Delta_{P}^{\vee}$. Now let $\lambda \in \mathcal{E}_{R}(\varphi)$ and for $P$ containing $R$, write $\lambda=\lambda_{R}^{P}+\lambda_{P}$ relative to the decomposition $\mathfrak{A}_{R}=\mathfrak{A}_{R}^{P} \oplus \mathfrak{A}_{P}$. By our hypothesis, $\left\langle\lambda_{P}, \varpi^{\vee}\right\rangle \neq 0$ for all $\varpi^{\vee} \in \hat{\Delta}_{P}^{\vee}$ and hence $\lambda_{P}$ is non-degenerate with respect to $\mathcal{C}_{P}$. Similarly, $\left\langle\lambda_{R}^{P}, \alpha^{\vee}\right\rangle \neq 0$ for all $\alpha \in \Delta_{R}^{P}$ and hence $\lambda_{R}^{P}$ is non-degenerate with respect to $\widehat{\mathcal{C}}_{R}^{P}$. Since $\mathcal{C}_{P} \times \widehat{\mathcal{C}}_{R}^{P} \subset \widehat{\mathcal{C}}_{R}$ for all $P$, we may apply Lemma 6 to conclude that for any polynomial $Q(X)$,

$$
-(-1)^{d(R)-d(G)} \int_{\mathfrak{A}_{R}}^{\#} Q(X) e^{\langle\lambda, 2 X\rangle} \hat{\tau}_{R}(2 X-T) d X
$$


is equal to

$$
\sum_{\substack{P \\ R \subset P \neq G}}(-1)^{d(R)-d(P)} \int_{\mathfrak{A}_{R}}^{\#} Q(X) e^{\langle\lambda, 2 X\rangle} \hat{\tau}_{R}^{P}(2 X-T) \tau_{P}(2 X-T) d X .
$$

It follows that (39) is equal to

$$
-\sum_{R \neq G}(-1)^{d(R)-d(G)} \int_{R(F) \backslash G(\mathbb{A})^{1}}^{*} \varphi_{R_{E}}(g) \hat{\tau}_{R}(H(g)-T) d g
$$

and this gives the equality of the theorem.

We now prove the equality of (36) and (37). Write the constant term $\varphi_{P_{E}}$ as a sum

$$
\varphi_{P_{E}}(n a m k)=\sum_{j=1}^{r} Q_{j}\left(H_{E}(a)\right) \psi_{j}(a m k)
$$

for $n \in N\left(\mathbb{A}_{E}\right), a \in A_{P_{E}}, m \in M\left(\mathbb{A}_{E}\right)^{1}$ and $k \in \mathbf{K}_{E}$, where the $Q_{j}$ are polynomials and $\psi_{j} \in \mathcal{A}_{P_{E}}(G)$ satisfies

$$
\psi_{j}(a g)=e^{\left\langle\lambda_{j}+\rho_{P}, H_{E}(a)\right\rangle} \psi_{j}(g)
$$

for some exponent $\lambda_{j} \in \mathfrak{A}_{P}^{*}$ for all $a \in A_{P_{E}}$. Then (36) is equal to

$$
\sum_{j=1}^{r}\left(\int_{\mathbf{K}_{F}} \int_{M \backslash M(\mathbb{A})^{1}} \Lambda_{m}^{T, M} \psi_{j}(m k) d m d k\right)\left(\int_{\mathfrak{A}_{P}}^{\#} Q_{j}(2 X) e^{\left\langle\lambda_{j}, 2 X\right\rangle} \tau_{P}(2 X-T) d X\right)
$$

where $\Lambda_{m}^{T, M}$ denotes the mixed truncation with respect to $M$. Using our induction hypothesis, we may write (36) as the sum over $j$ and $R \subset P$ of $(-1)^{d(R)-d(P)}$ times

$$
\left(\int_{\mathbf{K}_{F}} \int_{R_{M}(F) \backslash M(\mathbb{A})^{1}}^{*}\left(\psi_{j}\right)_{\left(M_{R}\right)_{E}}(m k) \hat{\tau}_{R}^{P}\left(H_{E}(m)-T\right) d m d k\right)
$$

times

$$
\left(\int_{\mathfrak{A}_{P}}^{\#} Q_{j}(2 X) e^{\left\langle\lambda_{j}, 2 X\right\rangle} \tau_{P}(2 X-T) d X\right)
$$

where $R_{M}=R \cap M$. Choose a decomposition of type (40) for the constant term $\left(\psi_{j}\right)_{R_{M}}$ :

$$
\left(\psi_{j}\right)_{\left(R_{M}\right)_{E}}(n a m k)=\sum_{j=1}^{r} P_{j, \ell}\left(H_{E}(a)\right) \psi_{j, \ell}(a m k)
$$

for $n \in\left(N_{R} \cap M\right)\left(\mathbb{A}_{E}\right), a \in A_{R} \cap M\left(\mathbb{A}_{E}\right)^{1}, m \in M_{R}\left(\mathbb{A}_{E}\right)^{1}, k \in \mathbf{K}_{E}$, where the $\psi_{j, \ell}$ satisfy

$$
\psi_{j, \ell}(a g)=e^{\left\langle\lambda_{j, \ell}+\rho_{R}^{P}, H_{E}(a)\right\rangle} \psi_{j, \ell}(g)
$$

for some exponents $\lambda_{j, \ell} \in\left(\mathfrak{A}_{R}^{P}\right)^{*}$. Then we may write (41) as a sum over $\ell$ of

$$
\int_{\mathbf{K}_{F}} \int_{M_{R}(F) \backslash M_{R}(\mathbb{A})^{1}}^{*} \psi_{j, \ell}(m k) d m d k \int_{\mathfrak{A}_{R}^{P}}^{\#} P_{j, \ell}(2 X) e^{\left\langle\lambda_{j, \ell}, 2 X\right\rangle} \hat{\tau}_{R}^{P}(2 X-T) d X .
$$


By (11), we may combine the \#-integrals over $\mathfrak{A}_{R}^{P}$ and $\mathfrak{A}_{P}$ into a single \#-integral over $\mathfrak{A}_{R}$ and we see that

$$
\begin{array}{r}
\int_{\mathbf{K}_{F}} \int_{R_{M}(F) \backslash M(\mathbb{A})^{1}}^{*}\left(\psi_{j}\right)_{\left(M_{R}\right)_{E}}(m k) \hat{\tau}_{R}^{P}\left(H_{E}(m)-T\right) d m d k \\
\times\left(\int_{\mathfrak{A}_{P}}^{\#} Q_{j}(2 X) e^{\left\langle\lambda_{j}, 2 X\right\rangle} \tau_{P}(2 X-T) d X\right)
\end{array}
$$

is equal to the sum over $\ell$ of

$$
\begin{aligned}
\int_{\mathbf{K}_{F}} \int_{M_{R}(F) \backslash M_{R}(\mathbb{A})^{1}}^{*} \psi_{j, \ell}(m k) d m d k & \\
& \times \int_{\mathfrak{A}_{R}}^{\#} P_{j, \ell}(2 X) Q_{j}(2 X) e^{\left\langle\mu_{j, \ell}, 2 X\right\rangle} \hat{\tau}_{R}^{P}(2 X-T) \tau_{P}(2 X-T) d X
\end{aligned}
$$

where $\mu_{j, \ell}=\lambda_{j}+\lambda_{j, \ell}$, and this equals

$$
\int_{R(F) \backslash H(\mathbb{A})^{1}}^{*} Q_{j}\left(H_{P_{E}}(g)\right)\left(\psi_{j}\right)_{R_{M}}(g) \hat{\tau}_{R}^{P}\left(H_{E}(g)-T\right) \tau_{P}\left(H_{E}(g)-T\right) d g .
$$

Summing over $j$ gives

$$
\int_{R(F) \backslash H(\mathbb{A})^{1}}^{*} \varphi_{R}(g) \hat{\tau}_{R}^{P}\left(H_{E}(g)-T\right) \tau_{P}\left(H_{E}(g)-T\right) d g .
$$

This shows that (36) is equal to

$$
\sum_{R \subset P}(-1)^{d(R)-d(P)} \int_{R(F) \backslash H(\mathbb{A})^{1}}^{*} \varphi_{R}(g) \hat{\tau}_{R}^{P}\left(H_{E}(g)-T\right) \tau_{P}\left(H_{E}(g)-T\right) d g,
$$

as required.

Corollary 11. For all $\varphi \in \mathcal{A}(G)^{* *}$, the period integral

$$
\int_{H(F) \backslash H(\mathbb{A})^{1}} \Lambda_{m}^{T} \varphi(h) d h
$$

is an exponential polynomial function of the truncation parameter $T$ whose exponents are contained in those of $\varphi$.

Proof. According to the definition of

$$
\int_{P \backslash H(\mathbb{A})^{1}}^{*} \varphi_{P_{E}}(h) \hat{\tau}_{P}\left(H_{E}(h)-T\right) d h,
$$

the only dependence on $T$ is through \#-integrals of the form

$$
\int_{\mathfrak{A}_{P}}^{\#} Q_{j}(2 X) e^{\left\langle\lambda_{j}, 2 X\right\rangle} \hat{\tau}_{P}(2 X-T) d X .
$$

The assertion now follows from Lemma 5.

In fact, one can prove the corollary, without any restrictions on $\varphi$ by using the relation (33). 
9. Eisenstein series. Our next goal is to verify that Theorem 10 applies to cuspidal Eisenstein series. To that end, we fix some notation and definitions that will be used in the rest of the article. We work with a reductive group $G$ over a field $F$.

Let $P=M N$ be a proper parabolic subgroup and let $\sigma$ be an automorphic subrepresentation of $L^{2}\left(M \backslash M(\mathbb{A})^{1}\right)$. Let $\mathcal{A}_{P}(G)_{\sigma}$ be the subspace of functions $\varphi \in$ $\mathcal{A}_{P}(G)$ such that $\varphi$ is left-invariant under $A_{P}$ and for all $k \in \mathbf{K}$, the function $m \longmapsto$ $\varphi(m k)$ belongs to the space of $\sigma$. For $\varphi \in \mathcal{A}_{P}(G)_{\sigma}$ and $\lambda \in \mathfrak{A}_{P}^{*}$ we write $E(g, \varphi, \lambda)$ for the Eisenstein series which is given, in its domain of absolute convergence, by the infinite series

$$
E(g, \varphi, \lambda)=\sum_{\gamma \in P \backslash G} \varphi(\gamma g) e^{\left\langle\lambda+\rho_{P}, H_{P}(\gamma g)\right\rangle} .
$$

Let $N_{G(F)}\left(A_{0}\right)$ be the normalizer of $A_{0}$ in $G(F)$ and let

$$
\Omega=N_{G(F)}\left(A_{0}\right) / C_{G(F)}\left(A_{0}\right)
$$

be the Weyl group of $G$. Recall that a parabolic subgroup $Q$ is said to be associate to $P$ if $M_{Q}$ is conjugate to $M_{P}$ under $\Omega$. If $Q$ is associate to $P$, let $\Omega(P, Q)$ be the set of maps $A_{P} \longrightarrow A_{Q}$ obtained by restriction of elements $w \in \Omega$ such that $w M_{P} w^{-1}=M_{Q}$.

Suppose that $Q$ is associate to $P$ and let $w$ be an element of $\Omega(P, Q)$ with representative $\widetilde{w}$ in $N_{G(F)}\left(A_{0}\right)$. We define the standard intertwining operator

$$
M(w, \lambda) \varphi(g)=e^{-\left\langle w \lambda+\rho_{Q}, H_{Q}(g)\right\rangle} \int_{N_{w}(\mathbb{A}) \backslash N_{Q}(\mathbb{A})} \varphi\left(\widetilde{w}^{-1} n g\right) e^{\left\langle\lambda+\rho_{P}, H_{P}\left(\widetilde{w}^{-1} n g\right)\right\rangle} d n
$$

where $N_{w}=N_{Q} \cap \widetilde{w} N \widetilde{w}^{-1}$. The operator $M(w, \lambda)$ depends on $w$ but not on the choice of representative $\widetilde{w}$.

Assume that $\sigma$ is cuspidal. Then the constant term $E_{Q}(g, \varphi, \lambda)$ relative to a parabolic subgroup $Q$ has a simple expression. If $Q$ does not contain an associate of $P$, then $E_{Q}(g, \varphi, \lambda)$ is identically zero. If $Q$ is associate to $P$, then

$$
E_{Q}(g, \varphi, \lambda)=\sum_{w \in \Omega(P, Q)} M(w, \lambda) \varphi(g) e^{\left\langle w \lambda+\rho_{Q}, H_{Q}(g)\right\rangle} .
$$

On the other hand, if $Q$ properly contains an associate of $P$, then [A2]

$$
E_{Q}(g, \varphi, \lambda)=\sum_{Q^{\prime}} \sum_{\substack{w \in \Omega\left(P, Q^{\prime}\right) \\ w^{-1} \alpha>0 \text { for } \alpha \in \Delta_{Q^{\prime}}^{Q}}} E^{Q}(g, M(w, \lambda) \varphi, w \lambda)
$$

where the sum is over the standard parabolic subgroups $Q^{\prime} \subset Q$ associate to $P$ and $E^{Q}(g, \psi, \lambda)$ denotes an Eisenstein series induced from $M_{Q^{\prime}}$ to $M_{Q}$ :

$$
E^{Q}(g, \psi, \lambda)=\sum_{\gamma \in Q^{\prime} \backslash Q} \psi(\gamma g) e^{\left\langle\lambda+\rho_{Q^{\prime}}, H_{Q^{\prime}}(\gamma g)\right\rangle} .
$$

We now return to the situation where $G=H_{/ E}$ where $E / F$ is quadratic as above. Consider a cuspidal Eisenstein series $E(g, \varphi, \lambda)$ on $G$. We shall check that $E(g, \varphi, \lambda)$ belongs to $\mathcal{A}(G)^{* *}$ for generic values of the parameter $\lambda$. For each $Q$ containing an associate of $P$, let $\mathcal{O}$ be the open set where $E^{Q}$ and the intertwining operators are regular. This is a complement of hyperplanes. In $\mathcal{O}$, the exponents of the Eisenstein series along a parabolic subgroup $Q$ are the restrictions to $\mathfrak{A}_{Q}$ of the linear forms $w \lambda$ with $P^{\prime}$ associate to $P$ and contained in $Q$ and $w \in \Omega\left(P, P^{\prime}\right)$ 
with $w^{-1} \Delta_{P^{\prime}}^{Q}>0$. Thus for $\lambda \in \mathcal{O}$ the Eisenstein series belongs to $\mathcal{A}(G)^{* *}$ if for every such $Q$ and such $w$, and all $\varpi^{\vee} \in\left(\hat{\Delta}^{\vee}\right)_{Q}^{Q^{\prime}}$ with $Q \subset Q^{\prime}$ we have

$$
\left\langle w \lambda, \varpi^{\vee}\right\rangle \neq 0 .
$$

If we denote this set of such $\lambda$ by $\mathcal{O}_{0}$, we see that the Eisenstein series $E(g, \varphi, \lambda)$ belongs to the space $\mathcal{A}(G)^{* *}$ for all $\lambda \in \mathcal{O}_{0}$.

Proposition 12. Let $E(g, \varphi, \lambda)$ be a cuspidal Eisenstein series. Then the period integral of a cuspidal Eisenstein series $\Pi^{G / H}(E(\varphi, \lambda))$ is a meromorphic function of $\lambda$ with hyperplane singularities. It is holomorphic on $\mathcal{O}_{0}$.

Proof. Assume that $E(\varphi, \lambda)$ is induced from $P_{E}=M_{E} N_{E}$. It will suffice to check the claim for each of the integrals

$$
\int_{Q \backslash H(\mathbb{A})^{1}}^{*} \Lambda_{m}^{T, Q} E_{Q}(h, \varphi, \lambda) \tau_{Q}\left(H_{E}(h)-T\right) d h .
$$

By (42), each of these integrals is a sum of terms of the form

$$
\int_{Q \backslash H(\mathbb{A})^{1}}^{*} \Lambda_{m}^{T, Q} E^{Q}(h, M(w, \lambda) \varphi, w \lambda) \tau_{Q}\left(H_{E}(h)-T\right) d h,
$$

which itself can be written as a product of

$$
\int_{\mathfrak{A}_{Q}}^{\#} e^{\langle w \lambda, 2 X\rangle} \tau_{Q}(2 X-T) d X
$$

and

$$
\int_{\mathbf{K}_{F} \times M_{Q} \backslash M_{Q}(\mathbb{A})^{1}} \Lambda_{m}^{T, Q} E^{Q}(m k, M(w, \lambda) \varphi, \lambda) d m d k .
$$

The first factor depends only on the projection $(w \lambda)_{Q}$ of $w \lambda$ to $\mathfrak{A}_{Q}$. It can be evaluated explicitly and is clearly meromorphic and holomorphic in $\mathcal{O}_{0}$. The second factor depends only on the projection $(w \lambda)_{0}^{Q}$ of $w \lambda$ to $\mathfrak{A}_{0}^{Q}$. The integrand is defined and varies analytically in $\mathcal{O}_{0}$. According to $[\mathrm{MW}]$, Lemma I.2.16, the rate of rapid decrease of Arthur's truncation $\Lambda^{T} \psi$ is majorized in terms of the rate of slow increase of finitely many derivatives of $\psi$ and hence in terms of the exponents of finitely many derivatives of $\psi$. The proof applies with little change to the mixed truncation and thus, similarly, the rate of rapid decrease of $\Lambda_{m}^{T, Q} \psi$ is similarly majorized in terms of the exponents of finitely many derivatives of $\psi$. In our case, these exponents vary analytically and we may therefore conclude that the integral (43) is uniformly convergent for $\lambda$ in a compact subset of $\mathcal{O}_{0}$. Hence (43) is analytic in $\mathcal{O}_{0}$.

Remark 1. It is not true in general that $\Pi^{G / H}(E(\varphi, \lambda))$ is analytic whenever $E(\varphi, \lambda)$ is analytic. For example, the computations in $\S 20$ show that for the Eisenstein series on $G L(2)$ induced from the trivial character and $\varphi \equiv 1$ we have $\Pi^{G / H}(E(\varphi, \lambda))=$ $\zeta_{F}(\lambda) / L\left(\lambda+1, \omega_{E / F}\right)$ up to a volume factor. The regularized period thus has a pole at $\lambda=0$. However $E(\varphi, 0) \equiv 0$ by the functional equation. 


\section{INTEGRAL OF AN AUTOMORPHIC FORM}

In this section, we describe how the considerations of the previous section can be carried over to the case that $H=G$. We omit most of the proofs since they are nearly identical, word for word, as those presented in the previous section.

Let $P$ be a parabolic subgroup of $G$ and let $f \in \mathcal{A}_{P}(G)$. Then there exists a finite set of distinct exponents $\mathcal{E}_{P}(f)=\left\{\lambda_{1}, \ldots, \lambda_{k}\right\}$ in $\mathfrak{A}_{P}^{*}$ such that

$$
f(n a m k)=\sum_{j=1}^{k} \phi_{j}(m k) \alpha_{j}(H(a)) e^{\left\langle\lambda_{j}+\rho_{P}, H(a)\right\rangle}
$$

for $n \in N(\mathbb{A}), a \in A_{P}, m \in M(\mathbb{A})^{1}$ and $k \in \mathbf{K}$, where for all $j, \alpha_{j}(X)$ is a polynomial, and $\phi_{j}(g)$ is an automorphic form in $\mathcal{A}_{P}(G)$ such that $\phi_{j}(a g)=\phi_{j}(g)$ for $a \in A_{P}$.

The function $\tau_{P}$ is the characteristic function of the cone spanned by the coweights in $\hat{\Delta}_{P}^{\vee}$. It follows that if

$$
\left\langle\lambda_{j}-\rho_{P}, \varpi^{\vee}\right\rangle \neq 0
$$

for all $\varpi^{\vee} \in \hat{\Delta}_{P}^{\vee}$ and $\lambda_{j} \in \mathcal{E}_{P}(f)$, then the integrals

$$
\int_{\mathfrak{A}_{P}}^{\#} \alpha_{j}(X) e^{\left\langle\lambda_{j}-\rho_{P}, X\right\rangle} \tau_{P}(X-T) d X
$$

are defined. Assuming this condition holds, we define the \#-integral

$$
\int_{P \backslash G(\mathbb{A})^{1}}^{\#} \Lambda^{T, P} f(g) \tau_{P}(H(g)-T) d g
$$

by the formula

$$
\sum_{j=1}^{k} \int_{\mathbf{K}}\left(\int_{M \backslash M(\mathbb{A})^{1}} \Lambda^{T, P} \phi_{j}(m k) d m\right)\left(\int_{\mathfrak{A}_{P}}^{*} \alpha_{j}(X) e^{\left\langle\lambda_{j}-\rho_{P}, X\right\rangle} \tau_{P}(X-T) d X\right) d k .
$$

We also denote this expression by $I_{P}^{T}(f)$. It coincides with the ordinary integral if $\Lambda^{T, P} f(g) \tau_{P}(H(g)-T)$ is integrable over $P \backslash G(\mathbb{A})^{1}$.

As before, for $\varphi \in \mathcal{A}(G)$, we write $\mathcal{E}_{P}(\varphi)$ for $\mathcal{E}_{P}\left(\varphi_{P}\right)$. Let

$$
\mathcal{A}(G)^{\prime}=\left\{\varphi \in \mathcal{A}(G):\left\langle\lambda-\rho_{P}, \varpi^{\vee}\right\rangle \neq 0 \text { for all } \varpi^{\vee} \in \hat{\Delta}_{P}^{\vee}, \lambda \in \mathcal{E}_{P}(\varphi), P \neq G\right\} .
$$

For $\varphi \in \mathcal{A}(G)^{\prime}$, we define the regularized integral

$$
\int_{G \backslash G(\mathbb{A})^{1}}^{*} \varphi(g) d g=\sum_{P \subset G} I_{P}^{T}\left(\varphi_{P}\right) .
$$

We also denote this integral by $I_{G}^{1}(\varphi)$. For $x \in G\left(\mathbb{A}_{f}\right)$, let $\rho(x)$ denote right translation by $x: \rho(x) \varphi(g)=\varphi(g x)$.

Theorem 13. (i) $I_{G}^{1}$ is independent of the choice of $T$.

(ii) The space $\mathcal{A}(G)^{\prime}$ is invariant under right translation by $G\left(\mathbb{A}_{f}\right)^{1}$ and $I_{G}^{1}$ defines an invariant linear functional on $\mathcal{A}(G)^{\prime}$. Explicitly, $I_{G}^{1}(\rho(x) \varphi)=I_{G}^{1}(\varphi)$ for all $x \in G\left(\mathbb{A}_{f}\right)$.

(iii) If $\varphi \in \mathcal{A}(G)$ is integrable over $G \backslash G(\mathbb{A})^{1}$, then $\varphi \in \mathcal{A}(G)^{\prime}$ and $I_{G}^{1}(\varphi)$ coincides with the ordinary integral of $\varphi$ over $G \backslash G(\mathbb{A})^{1}$. 
Proof. The proof is nearly identical to that of Theorem 9 and therefore we omit it. However, we merely note a slight modification required for part (iii). As in the prove of part (iii) of Theorem 9, it is necessary to check that if $\varphi \in \mathcal{A}(G)$ is integrable over $G \backslash G(\mathbb{A})^{1}$, then the integral

$$
\int_{P \backslash G(\mathbb{A})^{1}} \Lambda^{T, P} \varphi_{P}(g) \tau_{P}(H(g)-T) d g
$$

converges absolutely. Expand $\varphi_{P}$ as in (44). As shown in [MW], top of p. 50, for all $j$, there exists a parabolic subgroup $Q \subset P$ and a cuspidal exponent $\mu$ of $\varphi_{Q}^{\text {cusp }}$ such that $\lambda_{j}$ is equal to the restriction of $\mu$ to $\mathfrak{A}_{P}$ relative to the decomposition

$$
\mathfrak{A}_{Q}=\mathfrak{A}_{Q}^{P} \oplus \mathfrak{A}_{P} .
$$

According to Lemma I.4.11 of [MW], p. 75 , if $\varphi$ is square-integrable, the exponent $\mu$ can be written in the from $\sum_{\alpha \in \Delta_{Q}} x_{\alpha} \alpha$ with $x_{\alpha}<0$. This time, we modify the argument to show that if $\varphi$ is integrable, then

$$
\mu-\rho_{Q}=\sum_{\alpha \in \Delta_{Q}} x_{\alpha} \alpha
$$

with $x_{\alpha}<0$. The restriction of $\rho_{Q}$ to $\mathfrak{A}_{P}$ is $\rho_{P}$ and the set of non-zero restrictions of elements in $\Delta_{Q}$ to $\mathfrak{A}_{P}$ coincides with $\Delta_{P}$. It follows that

$$
\lambda_{j}-\rho_{P}=\sum_{\beta \in \Delta_{P}} y_{\beta} \beta
$$

with $y_{\beta}<0$ and hence

$$
\int_{\mathfrak{A}_{P}} Q_{j}(X) e^{\left\langle\lambda_{j}-\rho_{P}, X\right\rangle} \tau_{P}(X-T) d X
$$

converges absolutely. This is all that is required in order that the integral (45) be absolutely convergent.

10. Integral of a truncated automorphic form. We can now define a certain regularized integral for automorphic functions $f \in \mathcal{A}_{P}(G)$. Assume that $f$ has a decomposition as in (44) and define

$$
\int_{P \backslash G(\mathbb{A})^{1}}^{*} f(g) \hat{\tau}_{P}(H(g)-T) d g
$$

by

$$
\sum_{j=1}^{k} \int_{\mathbf{K}} \int_{M \backslash M(\mathbb{A})^{1}}^{*} \phi_{j}(m k) d m d k\left(\int_{\mathfrak{A}_{P}}^{\#} \alpha_{j}(X) e^{-\left\langle\rho_{P}, X\right\rangle} \hat{\tau}_{P}(X-T) d X\right) .
$$

This is well-defined provided that the following two conditions are satisfied:

(1) $\left\langle\lambda-\rho_{Q}, \varpi^{\vee}\right\rangle \neq 0$ for all $Q \subset P, \varpi^{\vee} \in\left(\hat{\Delta}^{\vee}\right)_{Q}^{P}, \lambda \in \mathcal{E}_{Q}(\varphi)$.

(2) $\left\langle\lambda-\rho_{P}, \alpha^{\vee}\right\rangle \neq 0$ for all $\alpha \in \Delta_{P}$, and $\lambda \in \mathcal{E}_{P}(\varphi)$.

Let $\mathcal{A}(G)^{\prime \prime}$ be the space of $\varphi \in \mathcal{A}(G)$ such that (1) (and then also (2)) is satisfied for any $P$. The following Theorem and Corollary follow by the same proofs as those of Theorem 10 and its Corollary. 
Theorem 14. For all $\varphi \in \mathcal{A}(G)^{\prime \prime}$,

is equal to

$$
\int_{G(F) \backslash G(\mathbb{A})^{1}} \Lambda^{T} \varphi(g) d g
$$

$$
\sum_{P \subset G}(-1)^{d(P)-d(G)} \int_{P(F) \backslash G(\mathbb{A})^{1}}^{*} \varphi_{P}(g) \hat{\tau}_{P}(H(g)-T) d g .
$$

Corollary 15. For all $\varphi \in \mathcal{A}(G)^{\prime \prime}$, the integral

$$
\int_{G(F) \backslash G(\mathbb{A})^{1}} \Lambda^{T} \varphi(g) d g
$$

is an exponential polynomial function of the truncation parameter $T$.

Again, the corollary can be proved directly and it applies to any $\varphi \in \mathcal{A}(G)$.

11. Integral of a truncated Eisenstein series. Let $E(g, \varphi, \lambda)$ be a cuspidal Eisenstein series. As in the proof of Proposition 12, one shows that $I_{G}^{1}(E(g, \varphi, \lambda))$ is defined for generic values of the parameter $\lambda$ and varies analytically in $\lambda$.

Lemma 16 (Bernstein's principle). Let $P=M N$ be a proper parabolic subgroup and let $\sigma$ be an irreducible cuspidal representation in $L^{2}\left(M(F) \backslash M(\mathbb{A})^{1}\right)$. Let $E(g, \varphi, \lambda)$ be an Eisenstein series where $\varphi \in \mathcal{A}_{P}(G)_{\sigma}$. Then

$$
I_{G}^{1}(E(g, \varphi, \lambda))=0
$$

for all $\lambda$ such that $E(g, \varphi, \lambda)$ and $I_{G}^{1}(E(g, \varphi, \lambda))$ are defined.

Proof. Suppose that $E(g, \varphi, \lambda)$ and its regularized integral are defined at $\lambda_{0}$. Then there exists an open set $\mathcal{O}$ containing $\lambda_{0}$ such that $I_{G}^{1}(E(g, \varphi, \lambda))$ is defined for all $\lambda \in \mathcal{O}$. The map $\varphi \longmapsto I_{G}^{1}(E(g, \varphi, \lambda))$ therefore defines a $G\left(\mathbb{A}_{f}\right)^{1}$-invariant functional on $\operatorname{Ind}_{P}^{G}\left(\sigma \otimes e^{\lambda}\right)$ for $\lambda \in \mathcal{O}$. Since there does not exist any such invariant functional for generic values of $\lambda$, the function $I_{G}^{1}(E(g, \varphi, \lambda))$ must vanish identically.

Corollary 17. Let $P=M N$ be a parabolic subgroup and let $(M, \sigma)$ be a cuspidal datum. Let $E(g, \varphi, \lambda)$ be an Eisenstein series where $\varphi \in \mathcal{A}_{P}(G)_{\sigma}$. Then

$$
\int_{G(F) \backslash G(\mathbb{A})^{1}} \Lambda^{T} E(g, \varphi, \lambda) d g
$$

is equal to zero if $P$ is not a minimal parabolic. If $P$ is minimal, then it is equal to

$$
v \sum_{w \in \Omega} \frac{e^{\langle w \lambda-\rho, T\rangle}}{\prod_{\alpha \in \Delta_{0}}\left\langle w \lambda-\rho, \alpha^{\vee}\right\rangle} \int_{M \backslash M^{1}(\mathbb{A}) \times \mathbf{K}} M(w, \lambda) \varphi(m k) d m d k
$$

where $v=\operatorname{vol}\left(\left\{\sum_{\alpha \in \Delta_{0}} a_{\alpha} \alpha^{\vee}: 0 \leq a_{\alpha}<1\right\}\right)$.

Proof. For $\lambda \in \mathfrak{A}_{P}^{*}$ generic, the integral

is equal to

$$
\int_{G(F) \backslash G(\mathbb{A})^{1}} \Lambda^{T} E(g, \varphi, \lambda) d g
$$

$$
\sum_{Q}(-1)^{d(Q)-d(G)} \int_{Q(F) \backslash G(\mathbb{A})^{1}}^{*} E_{Q}(g, \varphi, \lambda) \hat{\tau}_{Q}(H(g)-T) d g .
$$


Since $\varphi$ is cuspidal, $E_{Q}(g, \varphi, \lambda)$ vanishes identically unless $Q$ contains an associate of $P$. As a function of $m, E_{Q}(\operatorname{namk}, \varphi, \lambda)$ is a sum of Eisenstein series induced from parabolic subgroups associate to $P$ to $M_{Q}$. Therefore, by the previous lemma and its defining formula,

$$
\int_{Q(F) \backslash G(\mathbb{A})^{1}}^{*} E_{Q}(g, \varphi, \lambda) \hat{\tau}_{Q}(H(g)-T) d g
$$

vanishes unless $Q$ is associate to $P$. If $Q$ is associate to $P$, then $E_{Q}$ is a sum of cusp forms on $M_{Q}(\mathbb{A})$, and the integral of a cusp form over $M_{Q}(F) \backslash M_{Q}(\mathbb{A})^{1}$ is zero unless $Q$ is minimal. In this case, $Q=P$ and the only non-zero term is

$$
(-1)^{d(P)-d(G)} \int_{P(F) \backslash G(\mathbb{A})^{1}} E_{P}(g, \varphi, \lambda) \hat{\tau}_{P}(H(g)-T) d g .
$$

This is equal to

$$
\begin{aligned}
& (-1)^{d(P)-d(G)} \sum_{w \in \Omega}\left(\int_{\mathfrak{A}_{P}}^{\#} e^{\left\langle w \lambda-\rho_{P}, H\right\rangle} \hat{\tau}_{P}(H-T) d H\right) \\
& \times\left(\int_{M \backslash M^{1}(\mathbb{A}) \times \mathbf{K}} M(w, \lambda) \varphi(m k) d m d k\right) .
\end{aligned}
$$

The lemma follows from the formula

$$
\left((-1)^{d(P)-d(G)} \int_{\mathfrak{A}_{P}}^{\#} e^{\left\langle w \lambda-\rho_{P}, H\right\rangle} \hat{\tau}_{P}(H-T) d H\right)=v \frac{e^{\left\langle w \lambda-\rho_{P}, T\right\rangle}}{\prod_{\alpha \in \Delta_{P}}\left\langle w \lambda-\rho_{P}, \alpha^{\vee}\right\rangle} .
$$

12. General inner product formula. The formula in Theorem 14 can be extended to a formula for the inner product

$$
\int_{G(F) \backslash G(\mathbb{A})^{1}} \Lambda^{T} \psi(g) \Lambda^{T} \varphi(g) d g
$$

where $\psi, \varphi \in \mathcal{A}(G)$. Recall that $\Lambda^{T}$ is a self-adjoint projection [A2] and therefore (47) is equal to

$$
\int_{G(F) \backslash G(\mathbb{A})^{1}} \psi(g) \Lambda^{T} \varphi(g) d g .
$$

Define a bilinear form on $\mathcal{A}(G)$ by

$$
B_{G}(\psi, \varphi)=\sum_{P \subset G} \int_{P \backslash G(\mathbb{A})^{1}}^{\#} \psi_{P}(g) \Lambda^{T, P} \varphi_{P}(g) \tau_{P}(H(g)-T) d g .
$$

The \#-integral is defined, as before, as a sum of products of absolutely convergent integrals over $M(\mathbb{A})^{1} \times \mathbf{K}$ and \#-integrals over $\mathfrak{A}_{P}$. This reduces to the usual inner product if either $\varphi$ or $\psi$ is cuspidal. In general, $B_{G}(\psi, \varphi)$ is well defined if, for all $P, \lambda \in \mathcal{E}_{P}(\varphi)$, and $\mu \in \mathcal{E}_{P}(\psi)$, we have $\left\langle\lambda+\mu, \varpi^{\vee}\right\rangle \neq 0$ for all $\varpi^{\vee} \in \hat{\Delta}_{P}^{\vee}$. One shows, as in Section IV that $B_{G}$ is invariant under $G\left(\mathbb{A}_{f}\right)$ and independent of $T$.

We use $B_{G}$ to define a regularized inner product

$$
\int_{P(F) \backslash G(\mathbb{A})^{1}}^{*} \psi(g) \varphi(g) \hat{\tau}_{P}(H(g)-T) d g
$$


for $\varphi, \psi \in \mathcal{A}_{P}(G)$. Suppose that

$$
\varphi(n a m k)=\sum_{i} Q_{i}\left(H_{P}(a)\right) e^{\left\langle\lambda_{i}+\rho_{P}, H_{P}(a)\right\rangle} \varphi_{i}(m k)
$$

and

$$
\psi(n a m k)=\sum_{j} R_{j}\left(H_{P}(a)\right) e^{\left\langle\mu_{j}+\rho_{P}, H_{P}(a)\right\rangle} \psi_{j}(m k)
$$

in the usual notation. Then we set (48) equal to

$$
\sum_{i, j}\left(\int_{\mathbf{K}} B_{M_{P}}^{1}\left(\psi_{j}(\bullet k), \varphi_{i}(\bullet k)\right) d k\right)\left(\int_{\mathfrak{A}_{P}}^{\#} Q_{i}(X) R_{j}(X) e^{\left\langle\lambda_{i}+\mu_{j}, X\right\rangle} \hat{\tau}_{P}(X-T) d X\right) .
$$

This is well-defined provided that

$\left(1^{\prime}\right)$ For all $Q \subset P, \lambda^{\prime} \in \mathcal{E}_{Q}(\varphi)$, and $\mu^{\prime} \in \mathcal{E}_{Q}(\psi)$, we have $\left\langle\lambda^{\prime}+\mu^{\prime}, \varpi^{\vee}\right\rangle \neq 0$ for all $\varpi^{\vee} \in\left(\hat{\Delta}^{\vee}\right)_{Q}^{P}$.

$\left(2^{\prime}\right)\left\langle\lambda+\mu, \alpha^{\vee}\right\rangle \neq 0$ for all $\alpha \in \Delta_{P}$.

Again, by arguments parallel to those in the proof of Theorem 10, we obtain the following

Theorem 18. Let $\psi, \varphi \in \mathcal{A}(G)$. Suppose that $\psi_{P}$ and $\varphi_{P}$ satisfy condition (1') (and then also $\left(2^{\prime}\right)$ ) for all $P$. Then the integral

$$
\int_{P(F) \backslash G(\mathbb{A})^{1}}^{*} \psi_{P}(g) \varphi_{P}(g) \hat{\tau}_{P}(H(g)-T) d g
$$

is well-defined for all $P$ and

$$
\int_{G(F) \backslash G(\mathbb{A})^{1}} \Lambda^{T} \psi(g) \Lambda^{T} \varphi(g) d g
$$

is equal to

$$
\sum_{P \subset G}(-1)^{d(P)-d(G)} \int_{P(F) \backslash G(\mathbb{A})^{1}}^{*} \psi_{P}(g) \varphi_{P}(g) \hat{\tau}_{P}(H(g)-T) d g .
$$

This theorem may be regarded as a generalization of the Langlands inner product formula. When applied to cuspidal Eisenstein series $E(g, \varphi, \lambda)$ and $E(g, \psi, \lambda)$ (induced from parabolics $P$ and $P^{\prime}$ ) we obtain the formula

$$
\begin{aligned}
& \int_{G(F) \backslash G(\mathbb{A})^{1}} \Lambda^{T} E(g, \psi, \lambda) \Lambda^{T} E(g, \varphi, \mu) d g \\
= & \sum_{Q}(-1)^{d(Q)-d(G)} \int_{Q(F) \backslash G(\mathbb{A})^{1}}^{*} E_{Q}(g, \psi, \lambda) E_{Q}(g, \varphi, \mu) \hat{\tau}_{Q}(H(g)-T) d g .
\end{aligned}
$$

As noted before, the constant term $E_{Q}(g, \psi, \lambda)$ vanishes unless $Q$ contains an associate of $P^{\prime}$. On the other hand, if $Q$ properly contains an associate of $P$ or $P^{\prime}$, then

$$
\int_{Q(F) \backslash G(\mathbb{A})^{1}}^{*} E_{Q}(g, \psi, \lambda) E_{Q}(g, \varphi, \mu) \hat{\tau}_{Q}(H(g)-T) d g=0
$$

by formula (42) and a variant of Bernstein's principle, viz., non-existence of an invariant pairing, either locally or globally, between $\operatorname{Ind}_{P}^{Q}\left(\sigma^{\prime} \otimes e^{\lambda}\right)$ and $\operatorname{Ind}_{P^{\prime}}^{Q}\left(\tau^{\prime} \otimes e^{\mu}\right)$ for generic $\lambda, \mu \in \mathfrak{A}_{P^{\prime}}$ and any representations $\sigma^{\prime}, \tau^{\prime}$ of $M_{P^{\prime}}$. Therefore (49) reduces 
to a sum over parabolic subgroups $Q$ associate to $P$. Direct calculation of the *integral using formula (42) for the constant terms $E_{Q}(g, \psi, \lambda)$ immediately yields the Langlands inner product formula [A2], Lemma 4.2.

\section{Regularized Periods of a Cuspidal Eisenstein SERIES on $G L(n)$}

For the rest of the paper, $H=G L(n)_{/ F}$ and $G=G L(n)_{/ E}$. Our goal in this section is to evaluate

$$
\int_{H(F) \backslash H(\mathbb{A})^{1}} \Lambda_{m}^{T} E(h, \varphi, \lambda) d h
$$

for the case of cuspidal Eisenstein series.

13. Weyl groups and double cosets. We take this opportunity to fix some notation that will be used from now on. Let $B=T U$ be the standard Borel subgroup of upper-triangular matrices of $H, T$ the diagonal subgroup, and $U$ the unipotent radical of $B$. We identify the Weyl groups $N_{H}(T) / T$ and $N_{G}\left(T_{E}\right) / T_{E}$ of $H$ and $G$, respectively and set $\Omega=N_{H}(T) / T=N_{G}\left(T_{E}\right) / T_{E}$ with length function $\ell$. The group $\Omega$ is isomorphic to the symmetric group $S_{n}$ and is naturally identified with the group of permutation matrices in $H$ or $G$ (matrices whose entries are 0 or 1 , with a single 1 in each row and column). For any (standard) parabolic subgroup $P=M N$ of $H$, we let $\Omega_{M}=N_{M}(T) / T$ be the Weyl group of the (standard) Levi factor $M$. In this case, $M$ consists of block diagonal matrices with blocks of size $n_{1}, \ldots, n_{r}$ for some partition $n=n_{1}+\cdots+n_{r}$ and is thus isomorphic to a product $G L\left(n_{1}\right) \times \cdots \times G L\left(n_{r}\right)$.

Let $\Omega(M)$ be the set of elements $w \in \Omega$ such that $M^{\prime}=w M w^{-1}$ is again a standard Levi subgroup and $w$ is of minimal length in the class $w \Omega_{M}$. This latter condition is satisfied if and only if $w \Delta_{0}^{M}=\Delta_{0}^{M^{\prime}}$. Explicitly, an element $w \in \Omega(M)$ is represented by a unique permutation matrix that shuffles the diagonal blocks of $M$ without causing any internal change within each block. The permutation matrix itself is built out of blocks of size $n_{j} \times n_{j}$ (not necessarily along the diagonal) which are either the identity matrix or the zero matrix.

If $M^{\prime}$ is another standard Levi subgroup, let

$$
\Omega\left(M, M^{\prime}\right)=\left\{w \in \Omega(M): w M w^{-1}=M^{\prime}\right\} .
$$

We also set

$$
\Omega^{M}=\left\{w \in \Omega: w M w^{-1}=M\right\} .
$$

Then $\Omega(M, M)$ is a subgroup of $\Omega^{M}$ and we have

$$
\Omega^{M}=\Omega(M, M) \ltimes \Omega_{M} .
$$

Let $\Omega_{2}=\left\{\xi \in \Omega: \xi^{2}=e\right\}$ and let

$$
\Omega_{2}(M, M)=\left\{w \in \Omega(M, M): w^{2}=e\right\}
$$

be the set of involutions in $\Omega(M, M)$. If $\xi \in \Omega_{2}(M, M)$, we denote the \pm 1 eigenspaces of $\xi$ in $\mathfrak{A}_{P}$ and $\mathfrak{A}_{P}^{*}$ by $\left(\mathfrak{A}_{P}\right)_{\xi}^{ \pm}$and $\left(\mathfrak{A}_{P}^{*}\right)_{\xi}^{ \pm}$, respectively. Let $P_{\xi}^{ \pm}$be the projections onto $\left(\mathfrak{A}_{P}^{*}\right)_{\xi}^{ \pm}$.

Lemma 19. 1. In any double coset $\kappa$ of $B(E) \backslash G(E) / H(F)$ there exists a representative $\eta$ so that $\eta \bar{\eta}^{-1} \in N_{G}\left(T_{E}\right)$ and its image in $\Omega$ depends only on $\kappa$. 
2. The map

$$
B(E) \backslash G(E) / H(F) \rightarrow \Omega
$$

defined above is injective and its image is $\Omega_{2}$.

3. Let $\xi \in \Omega_{2}$. There exists a constant $C$ such that

$$
\left\langle P_{\xi}^{-} \varpi, H_{E}(\eta)\right\rangle \leq C
$$

for all $\varpi \in \widehat{\Delta}_{0}$ and all $\eta \in G(E)$ such that $\eta \bar{\eta}^{-1}$ represents $\xi$ (i.e., $\eta \bar{\eta}^{-1} \in$ $\left.\xi T_{E}\right)$.

Proof. Part 1 is proved in [S], $\S 4$ (for the split case, but the proof carries over verbatim). Furthermore, $[\mathrm{S}]$ reduces part 2 to the following two statements, which follow from Hilbert's Theorem 90:

(a) Every $\xi \in \Omega_{2}$ can be represented by $g \bar{g}^{-1}$ for some $g \in G(E)$.

(b) If $\xi \in \Omega_{2}$, then the action of $\mathbb{Z} /(2)$ on $T_{E}$ by the involution $t \rightarrow \xi \bar{t} \xi^{-1}$ has trivial first cohomology.

To prove part 3, we observe that $\eta=\xi \bar{\eta}$ and hence if $\eta=a n k$, then

$$
H_{E}(\eta)=H_{E}(\xi \bar{\eta})=\xi H_{E}(\bar{\eta})+H_{E}(\xi \bar{n}) .
$$

We have $H_{E}(\bar{\eta})=H_{E}(\eta)$ and thus $P_{\xi}^{-} H_{E}(\eta)=\frac{1}{2} H_{E}(\xi n)$. The result follows from the fact that $\left\langle\varpi, H_{E}(\xi n)\right\rangle$ is bounded for all $\varpi \in \hat{\Delta}_{0}$.

We now describe $P(E) \backslash G(E) / H(F)$ for general $P$. For any such double coset, take a representative $\eta$ so that $\eta \bar{\eta}^{-1}$ represents $w \in \Omega_{2}$. Map $w$ to its reduced representative in $\Omega_{M} \backslash \Omega / \Omega_{M}$. Denote by $\iota_{P}$ the resulting map from $P(E) \backslash G(E) / H(F)$ to $\Omega_{M} \backslash \Omega / \Omega_{M}$. We identify $\Omega_{M} \backslash \Omega / \Omega_{M}$ with ${ }^{M} \Omega^{M}$ - the set of left and right $\Omega_{M}$-reduced elements in $\Omega$. Let ${ }^{M} \Omega_{2}^{M}$ be the set of involutions in ${ }^{M} \Omega^{M}$. This corresponds to the double cosets in $\Omega_{M} \backslash \Omega / \Omega_{M}$ which contain an involution. Alternatively, these are double cosets $D$ which are self-inverse (i.e. $D^{-1}=D$ ). Indeed, if $\xi$ is the reduced representative in $\Omega_{M} w \Omega_{M}$, then $\xi^{-1}$ is the reduced representative of $\Omega_{M} w^{-1} \Omega_{M}$.

Proposition 20. $\iota_{P}$ is a bijection between $P(E) \backslash G(E) / H(F)$ and ${ }^{M} \Omega_{2}^{M}$.

Proof. Choosing another $\eta$ in the double coset changes $\eta \bar{\eta}^{-1}$ to $p \eta \bar{\eta}^{-1} \bar{p}^{-1}$ for some $p \in P(E)$. Since $P(E) \backslash G(E) / P(E) \longleftrightarrow \Omega_{M} \backslash \Omega / \Omega_{M}, \iota_{P}$ is well defined. Moreover, it is clear that the image of $\iota_{P}$ consists of the cosets of $\Omega_{M} \backslash \Omega / \Omega_{M}$ which contain an involution. Suppose that $P g H$ and $P g^{\prime} H$ have the same image under $\iota_{P}$. Let $\xi, \xi^{\prime} \in$ $\Omega_{2}$ be the image of $B g H$ and $B g^{\prime} H$ respectively under $\iota_{B}$. By assumption $\xi^{\prime} \in$ $\Omega_{M} \xi \Omega_{M}$. We first prove that $\xi^{\prime}$ can be obtained from $\xi$ by successively performing two kinds of operations: conjugation by an element of $\Omega_{M}$ and multiplying by a simple reflection inside $M$ which commutes with the involution. We may suppose, to begin with, that $\xi^{\prime} \in{ }^{M} \Omega_{2}^{M}$. If $\xi \neq \xi^{\prime}$, then $\xi$ is not reduced and there exists $\alpha \in \Delta^{P}$ so that $\xi \alpha<0$, that is, $\ell\left(\xi s_{\alpha}\right)=\ell(\xi)-1$. If $\xi \alpha \neq-\alpha$, then $\left(\xi s_{\alpha}\right)^{-1} \alpha<0$ and hence $\ell\left(s_{\alpha} \xi s_{\alpha}\right)=\ell(\xi)-2$. If $\xi \alpha=-\alpha$, then $\xi^{-1} s_{\alpha} \xi=s_{\xi \alpha}=s_{\alpha}$ and $\xi=\left(\xi s_{\alpha}\right) s_{\alpha}$. In any case, we can reduce the length of $\xi$ by the operations described above. Next, we show that these operations can be realized by $\xi \mapsto p \xi \bar{p}^{-1}$ for $p \in P(E)$. In other words, if $\eta \bar{\eta}^{-1}$ represents $\xi \in \Omega_{2}$ and $\xi^{\prime \prime} \in \Omega_{2}$ is obtained by one of the operations above, then $\xi^{\prime \prime}$ can be represented by $p \eta \bar{\eta}^{-1} \bar{p}^{-1}$. This is evident for the first operation. For the second one, suppose that $s_{\alpha}$ commutes 
with $\xi$ with $\alpha \in \Delta_{0}^{M}$. Then $\xi \alpha= \pm \alpha$ and (possibly after interchanging $\xi$ and $\xi^{\prime \prime}=s_{\alpha} \xi$ ) we may assume that $\xi \alpha=\alpha$. We can also assume that $\eta \bar{\eta}^{-1}$ is actually equal to the permutation matrix of $\xi$ and hence centralizes the rank 1 subgroup $M_{\alpha}$ corresponding to the root $\alpha$. Choose $\eta_{\alpha} \in M_{\alpha} \subset M$ so that $\eta_{\alpha} \bar{\eta}_{\alpha}^{-1}$ represents $s_{\alpha}$. Then $\eta_{\alpha} \eta \bar{\eta}^{-1} \bar{\eta}_{\alpha}^{-1}=\eta \bar{\eta}^{-1} \eta_{\alpha} \bar{\eta}_{\alpha}^{-1}$ represents $\xi^{\prime \prime}$. Thus, there exists $g^{\prime \prime} \in P g^{\prime} H$ such that $\iota_{B}\left(B g^{\prime \prime} H\right)=\xi$. By the injectivity part of Lemma 19, $B g H=B g^{\prime \prime} H$, hence injectivity of $\iota_{P}$.

For any double coset in $P(E) \backslash G(E) / H(F)$ choose a representative $\eta$ so that $\eta \bar{\eta}^{-1}$ is the permutation matrix $\xi \in{ }^{M} \Omega_{2}^{M}$. Let $G^{*}=\operatorname{Res}_{E / F} H$ and similarly for $P^{*}$. Define $F$-subgroups of $G^{*}$ by

$$
\begin{aligned}
H_{\eta} & =H \cap \eta^{-1} P^{*} \eta, \\
P_{\eta} & =\eta H \eta^{-1} \cap P^{*},
\end{aligned}
$$

and set $M_{\eta}=M^{*} \cap P_{\eta}, N_{\eta}=N^{*} \cap P_{\eta}$. We observe that

$$
\eta H \eta^{-1}=\left\{x \in G^{*}: \bar{x}=\xi^{-1} x \xi\right\} .
$$

Hence the groups $P_{\eta}, M_{\eta}, N_{\eta}$ depend only on $\xi$ and we sometimes denote them by $P_{\xi}, M_{\xi}, N_{\xi}$.

In general, $P_{\eta}$ need not equal $M_{\eta} N_{\eta}$. For example, consider the parabolic subgroup

$$
P=\left\{\left(\begin{array}{ccc}
* & * & * \\
* & * & * \\
0 & 0 & *
\end{array}\right)\right\}
$$

of $G L(3)$ and the involution $\xi=(23) \in{ }^{M} \Omega_{2}^{M}$. Then

$$
P_{\eta}(F)=\left\{\left(\begin{array}{ccc}
x & \beta & \bar{\beta} \\
0 & \alpha & 0 \\
0 & 0 & \bar{\alpha}
\end{array}\right): x \in F^{*} \text { and } \alpha, \beta \in E^{*}\right\}
$$

and $M_{\eta}(F)$ is the diagonal subgroup of $P_{\eta}(F)$, but $N_{\eta}=\{e\}$. However, we do have $P_{\eta}=M_{\eta} N_{\eta}$ if $\xi$ normalizes $M$. We now make the following definition.

Definition 1. A representative $\eta$ as above is called $P$-admissible if $\xi=\eta \bar{\eta}^{-1} \bmod T_{E}$ lies in $\Omega^{M}$. We also speak about an admissible double coset in $P(E) \backslash G(E) / H(F)$.

Lemma 21. Let $\eta$ be a representative of a double coset in $P(E) \backslash G(E) / H(F)$ such that $\xi=\eta \bar{\eta}^{-1}$ represents an element in ${ }^{M} \Omega_{2}^{M}$.

(1) In the case $P=B$, every $\eta$ is $B$-admissible.

(2) If $\eta$ is $P$-admissible, then $M_{\eta}$ is a twisted F-form of $M$ defined by the Galois action $m \mapsto \xi \bar{m} \xi^{-1}$.

(3) If $\eta$ is $P$-admissible, then $P_{\eta}=M_{\eta} N_{\eta}$ is a Levi decomposition of $P_{\eta}$. The center of $M_{\eta}$ is contained in the center of $M_{E}$.

(4) $\iota_{P}$ induces a bijection between the $P$-admissible double cosets in $P(E) \backslash G(E) / H(F)$ and $\Omega_{2}(M, M)$.

Proof. Part (1) is immediate from the definition since $\Omega^{T}=\Omega$. Part (2) is also immediate from the definition. Observe that

$$
P_{\eta}(F)=\left\{p \in P_{E}(E): \bar{p}=\xi^{-1} p \xi\right\} .
$$

Since $\xi \in \Omega^{M}$ normalizes $M$, it follows that $P \cap \xi N \xi^{-1}=N \cap \xi N \xi^{-1}$. Therefore, if $p=m n \in P_{\eta}(F)$ with $m \in M(E)$ and $n \in N(E)$, then $\bar{m}=\xi^{-1} m \xi$ and $\bar{n}=\xi^{-1} n \xi$. 
On the other hand, $M_{\eta}$ is the fixed point set of an involution and hence is reductive ([S]). This proves the first part of (3). The second claim follows from the fact that the center of $M_{\eta}(F)$ is contained in that of $M_{\eta}(E) \simeq M(E)$ since $M_{\eta}(F)$ is Zariski dense in $M_{\eta}(E)$. Finally, (4) follows from (50).

Remark 2. Explicitly, if $P$ corresponds to the partition $\left(n_{1}, \ldots, n_{r}\right)$, then the admissible double cosets correspond to involutions $\pi$ of $\{1, \ldots, r\}$ so that $n_{\pi(i)}=n_{i}$ and then $M_{\eta}(F) \simeq \prod_{\pi(i)=i} G L\left(n_{i}, F\right) \times \prod_{\pi(i)<i} G L\left(n_{i}, E\right)$.

\section{Invariant functionals on principal series representations.}

Proposition 22. Let $v$ be a place of $F$ where $E$ is inert and let $\tau$ be a character of the diagonal subgroup $T\left(E_{v}\right)$. Suppose there exists a non-zero $H_{v}$-invariant linear functional on the induced representation $\operatorname{Ind}_{B\left(E_{v}\right)}^{G\left(E_{v}\right)} \tau$. Then there exists $\eta \in$ $B(E) \backslash G(E) / H(F)$ such that the restriction of $\tau$ to $T_{\eta}\left(F_{v}\right)$ is trivial.

Proof. We drop $v$ from the notation in this proof. For $x \in G(E)$, denote by $\pi^{x}$ the representation obtained by conjugating $\pi$ by $x$. Let $\delta_{\eta}$ be the modulus function on $B_{\eta}$. According to the geometric lemma in [BZ], Theorem 5.2, the representation $\operatorname{Res}_{H(F)} \operatorname{Ind}_{B(E)}^{G(E)} \tau$ is "glued" from $\operatorname{ind}_{H_{\eta}(F)}^{H(F)}\left(\left.\delta_{B_{E}}^{1 / 2} \delta_{\eta}^{-1 / 2} \tau\right|_{B_{\eta}}\right)^{\eta}$ (induction with compact support) where $\eta$ ranges over representatives of $B(E) \backslash G(E) / H(F)$ as above. The modulus factors appear because we use normalized induction. We claim that $\delta_{B_{E}}^{1 / 2} \delta_{\eta}^{-1 / 2}=\delta_{\eta}^{1 / 2}$. Since $\delta_{B}=\delta_{B_{E}}^{1 / 2}$ on $B(F)$, it suffices to check that the restriction of $\delta_{B}$ to $B_{\eta}$ is $\delta_{\eta}$. Suppose that $\eta \bar{\eta}^{-1}$ represents $\xi \in \Omega_{2}$. Let $\mathfrak{u}_{\{\alpha, \xi \alpha\}}=\left\{x \in u_{\alpha}+u_{\xi \alpha}: A d\left(\eta \bar{\eta}^{-1}\right) x=\bar{x}\right\}$ where $\mathfrak{u}_{\alpha}$ is the root space of $\alpha$. Then $\operatorname{Lie}\left(U_{\eta}\right)$ decomposes as a direct sum of $\mathfrak{u}_{\{\alpha, \xi \alpha\}}$ over orbits $\{\alpha, \xi \alpha\} \subset \Phi^{+}$of $\xi$ and $\operatorname{dim}_{F} \mathfrak{u}_{\{\alpha, \xi \alpha\}}=|\{\alpha, \xi \alpha\}|$. We have $\operatorname{Lie}\left(A_{\eta}\right)=\left(\mathfrak{A}_{P}\right)_{\xi}^{+}$where $A_{\eta}$ is the split component of $T_{\eta}$ and thus for $t \in A_{\eta}$,

$$
\delta_{\eta}(t)=\prod_{\{\alpha, \xi \alpha\} \subset \Phi^{+}}|\operatorname{det} A d(t)|_{\mathfrak{u}_{\{\alpha, \xi \alpha\}} \mid}\left|=\prod_{\left\{\alpha \in \Phi^{+}: \xi \alpha>0\right\}}\right| \alpha(t) \mid .
$$

On the other hand, since $t \in A_{\eta}$ we have

$$
\prod_{\left\{\alpha \in \Phi^{+}: \xi \alpha<0\right\}}|\alpha(t)|=1
$$

because $|\alpha(t)||(-\xi \alpha)(t)|=1$. Thus:

$$
\delta_{\eta}(t)=\prod_{\alpha \in \Phi^{+}}|\alpha(t)|=\delta_{B}(t)
$$

as claimed. Thus, if $\operatorname{Ind}_{B(E)}^{G(E)} \tau$ has a non-zero $H(F)$-invariant functional, then at least one of the quotients $\operatorname{ind}_{H_{\eta}(F)}^{H(F)}\left(\delta_{\eta}^{1 / 2} \tau\right)^{\eta}$ must have a non-zero $H(F)$-invariant functional. In this case, the dual, which is isomorphic to $\operatorname{Ind}_{H_{\eta}(F)}^{H(F)}\left(\bmod _{H_{\eta}}^{-1 / 2}\left(\tau^{\eta}\right)^{-1}\right)$, has an $H(F)$-invariant vector. By Frobenius reciprocity this occurs if and only if the restriction of $\tau^{\eta}$ to $H_{\eta}(F)$ is trivial as claimed.

15. The regularized period. The next theorem describes the regularized period of a cuspidal Eisenstein series. We write $x \longrightarrow \bar{x}$ for the conjugation of $E$ over $F$ and if $\pi$ is a representation of $G\left(\mathbb{A}_{E}\right)$ or a Levi subgroup, we write $\bar{\pi}$ for the representation $g \longrightarrow \pi(\bar{g})$. Let $P$ be the parabolic subgroup of $H$ corresponding to the partition $\left(n_{1}, \ldots, n_{r}\right)$ of $n$. Assume that $P$ is a proper subgroup. If $\sigma=$ 
$\sigma_{1} \otimes \sigma_{2} \otimes \cdots \otimes \sigma_{r}$ is a representation of $M_{E}\left(\mathbb{A}_{E}\right)^{1}$ and $\lambda \in \mathfrak{A}_{P}^{*}$, we write $\sigma(\lambda)$ for the representation that extends $\sigma$ to $M_{E}\left(\mathbb{A}_{E}\right)$ by $\sigma(\lambda)(a m)=e^{\left\langle\lambda, H_{P_{E}}(a)\right\rangle} \sigma(m)$. We write $\sigma^{*}$ for the contragredient of $\sigma$.

Theorem 23. Let $\varphi \in \mathcal{A}_{P}(G)_{\sigma}$ where $\sigma=\sigma_{1} \otimes \sigma_{2} \otimes \cdots \otimes \sigma_{r}$ is a cuspidal representation of $M_{E}\left(\mathbb{A}_{E}\right)^{1}$, and let $E(\varphi, \lambda)=E(g, \varphi, \lambda)$ be the associated Eisenstein series. Suppose that $E(\varphi, \lambda)$ is regular at $\lambda=\lambda_{0}$ and that $\Pi^{G / H}\left(E\left(\varphi, \lambda_{0}\right)\right)$ is defined and non-zero. Then either $r=1$ and $\varphi=E\left(\varphi, \lambda_{0}\right)$ is a distinguished cusp form on $G\left(\mathbb{A}_{E}\right)$, or $r=2$ and $\sigma_{2}^{*}=\bar{\sigma}_{1}$. In this case, if $\left\langle\operatorname{Re} \lambda, \alpha^{\vee}\right\rangle>>0$ for the unique root $\alpha \in \Delta_{P}$, then the period is given by the following absolutely convergent integral:

$$
\Pi^{G / H}(E(\varphi, \lambda))=\int_{H_{\eta}(F) \backslash H(\mathbb{A})^{1}} e^{\left\langle\lambda+\rho_{P}, H_{P_{E}}(\eta h)\right\rangle} \varphi(\eta h) d h
$$

where $\eta \bar{\eta}^{-1}=\xi$ is the unique non-trivial element in $\Omega(M, M)$. Furthermore, $\Pi^{G / H}(E(\varphi, \lambda))$ extends to a meromorphic function of $\lambda$.

Remark 3. More precisely, it can be proved that the right hand side of the above formula converges absolutely in the domain $\left\langle\operatorname{Re} \lambda-\rho_{P}, \alpha^{\vee}\right\rangle>0([\mathrm{LP}])$.

Proof. We may suppose that $r>1$ and hence that $E(g, \varphi, \lambda)$ is a true Eisenstein series. If $\Pi^{G / H}\left(E\left(\varphi, \lambda_{0}\right)\right) \neq 0$, then $\Pi^{G / H}(E(\varphi, \lambda))$ is non-zero for generic $\lambda \in \mathfrak{A}_{P, \mathbb{C}}^{*}$. This implies that there exists a dense open set $\mathcal{O} \subset \mathfrak{A}_{P, \mathbb{C}}^{*}$ such that $\operatorname{Ind}_{P}^{G}(\sigma(\lambda))$ admits an $H\left(\mathbb{A}_{f}\right)^{1}$-invariant functional for all $\lambda \in \mathcal{O}$. Choose a place $v$ where $E_{v}$ is inert and $\sigma_{v}$ is unramified, induced from an unramified character $t \mapsto e^{\left\langle\alpha, H_{E}(t)\right\rangle}$ of $T_{E}$ for some $\alpha \in \mathfrak{A}_{0, \mathbb{C}}^{*}$. We observe that for $\xi \in \Omega_{2}, T_{\xi}=\left\{t \in T(E): \bar{t}=\xi t \xi^{-1}\right\}$ and $\mathfrak{A}_{\xi}^{+}$is the Lie algebra of the split component of $T_{\xi}$ modulo the center. From the previous proposition, we see that for all $\lambda \in \mathcal{O}$, there exists $\xi_{\lambda} \in \Omega_{2}$ such that $\left\langle\lambda+\alpha, \mathfrak{A}_{\xi_{\lambda}}^{+}\right\rangle=0$. Since $\Omega_{2}$ is a finite set, there exists $\xi \in \Omega_{2}$ and a subset $\mathcal{O}^{\prime} \subset \mathcal{O}$ with non-empty interior such that $\xi_{\lambda}=\xi$ for all $\lambda \in \mathcal{O}^{\prime}$. Therefore $\mathfrak{A}_{\xi}^{+}$is orthogonal to $\mathfrak{A}_{P}^{*}$ and hence $\mathfrak{A}_{P}^{*} \subset\left(\mathfrak{A}^{*}\right)_{\xi}^{-}$. We claim that $P$ must be a maximal parabolic subgroup of type $(m, m)$ where $m=\frac{n}{2}$ and $\xi$ is a Weyl group element that interchanges the two blocks. Indeed, $\xi$ acts by -1 on $\mathfrak{A}_{P}^{*}$ and therefore normalizes $M_{P}$, acting as a transposition on its blocks. But if $M_{P}$ has more than two blocks, then $\mathfrak{A}_{P}^{*} \cap\left(\mathfrak{A}^{*}\right)_{\xi}^{+} \neq 0$ since $\xi$ necessarily fixes certain non-scalar elements in the center of $M_{P}$.

Assume now that $P$ is of type $(m, m)$. Then its associate class consists only of $P$ itself. In particular, the constant term $E_{Q_{E}}(h, \varphi, \lambda)$ vanishes for all proper parabolic subgroups $Q \neq P$. Therefore

$$
\begin{aligned}
\Lambda_{m}^{T} E(h, \varphi, \lambda) & =E(h, \varphi, \lambda)-\sum_{\delta \in P \backslash H} E_{P_{E}}(\delta h, \varphi, \lambda) \hat{\tau}_{P}\left(H_{P_{E}}(\delta h)-T\right), \\
\Lambda_{m}^{T, P} E(h, \varphi, \lambda) & =E_{P_{E}}(h, \varphi, \lambda)
\end{aligned}
$$

and

$$
\begin{aligned}
\Pi^{G / H}(E(\varphi, \lambda))= & \int_{H \backslash H(\mathbb{A})^{1}} \Lambda_{m}^{T} E(h, \varphi, \lambda) d g \\
& +\int_{P \backslash H(\mathbb{A})^{1}}^{*} E_{P_{E}}(h, \varphi, \lambda) \hat{\tau}_{P}\left(H_{P_{E}}(h)-T\right) d h .
\end{aligned}
$$


Now assume that $\left\langle\operatorname{Re} \lambda, \alpha^{\vee}\right\rangle>>0$ where $\alpha$ is the unique root in $\Delta_{P}$. Using the formula

$$
E_{P_{E}}(h, \varphi, \lambda)=\varphi(h) e^{\left\langle\lambda+\rho_{P}, H_{P_{E}}(h)\right\rangle}+M(\xi, \lambda) \varphi(h) e^{\left\langle-\lambda+\rho_{P}, H_{P_{E}}(h)\right\rangle}
$$

we see that $\Lambda_{m}^{T} E(h, \varphi, \lambda)$ is equal to $I(h)+I I(h)+I I I(h)$ where

$$
\begin{gathered}
I(h)=\sum_{\delta \in P_{E} \backslash G-P \backslash H} \varphi(\delta h) e^{\left\langle\lambda+\rho_{P}, H_{P_{E}}(\delta h)\right\rangle}, \\
I I(h)=\sum_{\delta \in P \backslash H} \varphi(\delta h) e^{\left\langle\lambda+\rho_{P}, H_{P_{E}}(\delta h)\right\rangle}\left(1-\hat{\tau}_{P}\left(H_{P_{E}}(\delta h)-T\right)\right), \\
I I I(h)=-\sum_{\delta \in P \backslash H} M(\xi, \lambda) \varphi(\delta h) e^{\left\langle-\lambda+\rho_{P}, H_{P_{E}}(\delta h)\right\rangle_{\hat{\tau}_{P}}\left(H_{P_{E}}(\delta h)-T\right) .}
\end{gathered}
$$

The sum $I I(h)+I I I(h)$ is absolutely integrable over $H \backslash H(\mathbb{A})^{1}$ and the integral is equal to

$$
\begin{gathered}
\int_{P \backslash H(\mathbb{A})^{1}} \varphi(h) e^{\left\langle\lambda+\rho_{P}, H_{P_{E}}(h)\right\rangle}\left(1-\hat{\tau}_{P}\left(H_{P_{E}}(h)-T\right)\right) d h \\
-\quad \int_{P \backslash H(\mathbb{A})^{1}} M(\xi, \lambda) \varphi(h) e^{\left\langle-\lambda+\rho_{P}, H_{P_{E}}(h)\right\rangle_{\tau_{P}}\left(H_{P_{E}}(h)-T\right) d h .}
\end{gathered}
$$

Indeed, using the Iwasawa decomposition, we bound them by

$$
\int_{\mathfrak{A}_{P}} \int_{M(F) \backslash M(\mathbb{A})^{1} \times \mathbf{K}} e^{\left\langle\operatorname{Re} \lambda-\rho_{P}, H_{P_{E}}(X)\right\rangle}|\varphi(m k)|\left(1-\hat{\tau}_{P}\left(H_{P_{E}}(X)-T\right)\right) d X d m d k
$$

and

$$
\int_{\mathfrak{A}_{P}} \int_{M(F) \backslash M(\mathbb{A})^{1} \times \mathbf{K}} e^{\left\langle-\operatorname{Re} \lambda-\rho_{P}, H_{P_{E}}(X)\right\rangle}|M(\xi, \lambda) \varphi(m k)| \hat{\tau}_{P}\left(H_{P_{E}}(X)-T\right) d X d m d k .
$$

The integrals over $\mathfrak{A}_{P}$ are finite since $\left\langle\operatorname{Re} \lambda-\rho_{P}, \alpha^{\vee}\right\rangle>0$, as are the integrals over $M(F) \backslash M(\mathbb{A})^{1} \times \mathbf{K}$ since $\varphi$ and $M(\xi, \lambda) \varphi$ are integrable on $M(F) \backslash M(\mathbb{A})^{1}$. On the other hand, the relation (16) yields the equality

$$
\begin{aligned}
& \int_{P \backslash H(\mathbb{A})^{1}} \varphi(g) e^{\left\langle\lambda+\rho_{P}, H_{P_{E}}(h)\right\rangle}\left(1-\hat{\tau}_{P}\left(H_{P_{E}}(h)-T\right)\right) d h \\
= & -\int_{P \backslash H(\mathbb{A})^{1}}^{*} \varphi(g) e^{\left\langle\lambda+\rho_{P}, H_{P_{E}}(h)\right\rangle} \hat{\tau}_{P}\left(H_{P_{E}}(h)-T\right) d h,
\end{aligned}
$$

and so the contribution of $I I(h)+I I I(h)$ is equal to

$$
-\int_{P \backslash H(\mathbb{A})^{1}}^{*} E_{P_{E}}(h, \varphi, \lambda) \hat{\tau}_{P}\left(H_{P_{E}}(h)-T\right) d h .
$$

Now (53) shows that

$$
\Pi^{G / H}(E(\varphi, \lambda))=\int_{H(F) \backslash H(\mathbb{A})^{1}} I(h) d h,
$$

where we recall that

$$
I(h)=\sum_{\delta \in P_{E} \backslash G-P \backslash H} \varphi(\delta h) e^{\left\langle\lambda+\rho_{P}, H_{P_{E}}(\delta h)\right\rangle} .
$$


We know that $I(h)$ is absolutely integrable, but we do not yet know that the series

$$
|I|(h)=\sum_{\delta \in P_{E} \backslash G-P \backslash H}|\varphi(\delta h)| e^{\left\langle\operatorname{Re} \lambda+\rho_{P}, H_{P_{E}}(\delta h)\right\rangle}
$$

is integrable. We will show in fact that it is bounded. Assume that $\lambda$ is real for simplicity of notation, and set

$$
\begin{aligned}
I^{T}(h) & =\sum_{\delta \in P_{E} \backslash G-P \backslash H}|\varphi(\delta h)| e^{\left\langle\lambda+\rho_{P}, H_{P_{E}}(\delta h)\right\rangle_{\tau_{P}}\left(H_{P_{E}}(\delta h)-T\right),} \\
I_{T}(h) & =\sum_{\delta \in P_{E} \backslash G-P \backslash H}|\varphi(\delta h)| e^{\left\langle\lambda+\rho_{P}, H_{P_{E}}(\delta h)\right\rangle}\left(1-\hat{\tau}_{P}\left(H_{P_{E}}(\delta h)-T\right)\right) .
\end{aligned}
$$

Then $|I|(h)=I^{T}(h)+I_{T}(h)$. We shall bound $I^{T}(h)$ and $I_{T}(h)$ separately.

By [A1], Lemma 5.1, the number of $\delta \in P_{E} \backslash G$ such that $\hat{\tau}_{P}\left(H_{P_{E}}(\delta h)-T\right)=1$ is bounded by $C\|h\|^{N}$ for some constants $C, N>0$. To bound $I^{T}(h)$, it will therefore suffice to prove the following proposition.

Proposition 24. For any $M>0$ there exists a constant $K>0$ such that for $h$ in the Siegel domain $\mathcal{S}^{H}$ we have

$$
|\varphi(\delta h)| e^{\left\langle\lambda+\rho_{P}, H_{P_{E}}(\delta h)\right\rangle} \leq K\|h\|^{-M}
$$

if $\delta \in P_{E} \backslash G-P \backslash H$ and $\hat{\tau}_{P}\left(H_{P_{E}}(\delta h)-T\right)=1$.

We begin the proof of Proposition 24 with the following lemma. We write $C_{1}, C_{2}$, etc. for the positive constants that appear in the arguments below. Let $\left(\mathfrak{A}_{0}^{P}\right)^{*+}$ denote the positive Weyl chamber in $\left(\mathfrak{A}_{0}^{P}\right)^{*}$.

Lemma 25. There exists $\mu \in\left(\mathfrak{A}_{0}^{P}\right)^{*}$ and a constant $C$ with the following property. For all $h \in \mathcal{S}^{G} \cap H(\mathbb{A})^{1}$ and $\delta \in G(E)-P_{E} H$ such that $\delta h \in \mathcal{S}^{P_{E}}$, we have

$$
\left\langle\alpha, H_{P_{E}}(\delta h)\right\rangle \leq\left\langle\mu, H_{E}(\delta h)\right\rangle+C .
$$

Proof. Let $h \in \mathcal{S}^{G} \cap H(\mathbb{A})^{1}$ and $\delta \in G(E)-P_{E} H$. We do not assume at the outset that $\delta h \in \mathcal{S}^{P_{E}}$. However, multiplying $\delta$ on the left by $P_{E}(E)$ if necessary, we can use Proposition 20, to assume that $\delta \bar{\delta}^{-1} \in N_{G}\left(T_{E}\right)$ represents $\varrho \in{ }^{M} \Omega_{2}^{M}$. According to Lemma 19, (52), we have $\left\langle P_{\varrho}^{-} \varpi, H_{E}(\delta h)\right\rangle<C_{1}$ for some constant $C_{1}$ where $\hat{\Delta}_{P}=\{\varpi\}$. There exist a unique $a \in \mathbb{R}$ and $\nu \in\left(\mathfrak{A}_{0}^{P}\right)^{*}$ such that $P_{\varrho}^{-} \alpha=a \alpha+\nu$. Since $\delta \notin P_{E} H, \varrho$ does not lie in $\Omega_{M}$ and $a>0$ because $a=\frac{1}{2}\left\langle P_{\varrho}^{-} \alpha, \alpha^{\vee}\right\rangle=$ $\frac{1}{2}\left\langle P_{\varrho}^{-} \alpha, P_{\varrho}^{-} \alpha^{\vee}\right\rangle$. Also, $\left\langle P_{\varrho}^{-} \varpi, \beta^{\vee}\right\rangle=\frac{1}{2}\left\langle\varpi,-\varrho \beta^{\vee}\right\rangle \leq 0$ for any $\beta \in \Delta^{P}$, since $\varrho$ is reduced, so that $\nu \in-\left(\mathfrak{A}_{0}^{P}\right)^{*+}$. Using $\left\langle\alpha, H_{E}(\delta h)\right\rangle\left\langle a^{-1} C_{1}-a^{-1}\left\langle\nu, H_{E}(\delta h)\right\rangle\right.$ and setting $\kappa=-a^{-1} \nu$, we obtain

$$
e^{\left\langle\alpha, H_{P_{E}}(\delta h)\right\rangle} \leq C_{2} e^{\left\langle\kappa, H_{E}(\delta h)\right\rangle} .
$$

Now we observe that for any $\mu^{\prime} \in\left(\mathfrak{A}_{0}^{P}\right)^{*+}$ there exists $\mu^{\prime \prime} \in\left(\mathfrak{A}_{0}^{P}\right)^{*}$ such that for all $g \in G\left(\mathbb{A}_{E}\right)$ and $p \in P_{E}$ such that $p g \in \mathcal{S}^{P_{E}}$ we have

$$
e^{\left\langle\mu^{\prime}, H(g)\right\rangle} \leq C_{3} e^{\left\langle\mu^{\prime \prime}, H(p g)\right\rangle} .
$$


To prove this, write $p^{-1}=\delta^{\prime} u$ with $\delta^{\prime} \in M_{E}(E), u \in N(E)$ and $p g=n a m k$ where $m$ lies in the Siegel domain of $M_{E}^{1}$. Then

$$
e^{\left\langle\mu^{\prime}, H(g)\right\rangle}=e^{\left\langle\mu^{\prime}, H\left(\delta^{\prime} m\right)\right\rangle} \leq C_{4}\|m\|^{N} \leq C_{5} e^{\left\langle\mu^{\prime \prime}, H(m)\right\rangle}
$$

where the first inequality can be found in [A1], equation (5.2) in the proof of Lemma 5.1 , and the second inequality for some $\mu^{\prime \prime}$ is proved in [MW], p. 20 (vi). Now apply (55) with $\mu^{\prime}=\kappa$ and $g=\delta h$. Since $H_{P_{E}}(\delta h)=H_{P_{E}}(p \delta h)$, we obtain

$$
e^{\left\langle\alpha, H_{P_{E}}(p \delta h)\right\rangle} \leq C_{6} e^{\left\langle\mu^{\prime \prime}, H_{E}(p \delta h)\right\rangle}
$$

where $p \delta h \in \mathcal{S}^{P_{E}}$.

We now prove Proposition 24. Let $\mu$ be as in Lemma 25 and let $h \in \mathcal{S}^{G} \cap H(\mathbb{A})^{1}$ and $\delta \in G(E)-P_{E} H$. Since the conclusion of the proposition does not change if we replace $\delta$ by $p \delta$ for $p \in P_{E}$, we may assume that $\delta h$ belongs to $\mathcal{S}^{P_{E}}$. We get

$$
|\varphi(\delta h)| e^{\left\langle\lambda+\rho_{P}, H_{P_{E}}(\delta h)\right\rangle} \leq C_{7}|\varphi(\delta h)| e^{\langle\mu, H(\delta h)\rangle} .
$$

The function $m \longrightarrow \varphi(m k)$ is cuspidal for all $k \in \mathbf{K}$ and therefore rapidly decreasing on $M(E) \backslash M\left(\mathbb{A}_{E}\right)^{1}$. For any $\nu \in\left(\mathfrak{A}_{0}^{P}\right)^{*}$ there exists $C_{8}$ such that

$$
|\varphi(\delta h)| e^{\langle\mu, H(\delta h)\rangle} \leq C_{8} e^{\langle\nu, H(\delta h)\rangle}
$$

and thus

$$
|\varphi(\delta h)| e^{\left\langle\lambda+\rho_{P}, H_{P_{E}}(\delta h)\right\rangle} \leq C_{9} e^{\langle\nu, H(\delta h)\rangle} .
$$

Now, since $\delta h \in \mathcal{S}^{P_{E}}$,

$$
\left\langle\beta, H_{E}(\delta h)\right\rangle>\left\langle\beta, T_{0}\right\rangle \text { for } \beta \in \Delta_{0}^{M} .
$$

The hypothesis $\hat{\tau}_{P}\left(H_{P_{E}}(\delta h)-T\right)=1$ is equivalent to $\left\langle\alpha, H_{E}(\delta h)\right\rangle \geq\langle\alpha, T\rangle$ and therefore

$$
\|\delta h\| \leq C_{10} e^{\left\langle\zeta, H_{E}(\delta h)\right\rangle} e^{k\left\langle\alpha, H_{E}(\delta h)\right\rangle}
$$

for some $\zeta \in\left(\mathfrak{A}_{0}^{P}\right)^{*}$ and $k>0$. Again, using Lemma 25, given $N$, we may choose $\nu \in\left(\mathfrak{A}_{0}^{P}\right)^{*}$ so that

$$
e^{\langle\nu, H(\delta h)\rangle} \leq C_{11}\|\delta h\|^{-N} .
$$

By [MW], p. 21, (vii), there exists $C_{12}>0$ such that

$$
\|g\| \leq C_{12}\|\gamma g\|
$$

for all $g \in \mathcal{S}^{G}$ and $\gamma \in G(E)$, and hence

$$
|\varphi(\delta h)| e^{\left\langle\lambda+\rho_{P}, H_{P_{E}}(\delta h)\right\rangle} \leq C_{13}\|h\|^{-N} .
$$

This completes the proof of Proposition 24.

It remains to bound $I_{T}(h)$. In fact we will show that

$$
g \rightarrow \sum_{\delta \in P_{E} \backslash G}|\varphi(\delta g)| e^{\left\langle\lambda+\rho_{P}, H_{P_{E}}(\delta g)\right\rangle}\left(1-\hat{\tau}_{P}\left(H_{P_{E}}(\delta g)-T\right)\right)
$$


is bounded for $\lambda$ positive enough. We can assume that $g \in \mathcal{S}^{G}$. In analogy to (56), there exist $k_{1}>0$ and $\nu \in\left(\mathfrak{A}_{0}^{P}\right)^{*}$ such that

$$
\|\delta g\| \leq C_{14} e^{\left\langle\nu, H_{E}(\delta g)\right\rangle} e^{-k_{1}\left\langle\alpha, H_{P_{E}}(\delta g)\right\rangle}
$$

for all $\delta g \in \mathcal{S}^{P_{E}}$ and $\hat{\tau}_{P}\left(H_{P_{E}}(\delta g)-T\right)=0$. Since $\varphi$ is rapidly decreasing we infer that for any $N_{2}, k_{2}>0$

$$
|\varphi(\delta g)| e^{\left\langle\lambda+\rho_{P}, H_{P_{E}}(\delta g)\right\rangle}\left(1-\hat{\tau}_{P}\left(H_{P_{E}}(\delta g)-T\right)\right) \leq C_{15}\|\delta g\|^{-N_{2}} e^{-k_{2}\left\langle\alpha, H_{P_{E}}(\delta g)\right\rangle}
$$

provided that $\lambda$ is positive enough. For $x \in \mathbb{Z}, x<T$ is the number of $\delta$ such that $\delta g \in \mathcal{S}^{P_{E}}$ and $x \leq\left\langle\alpha, H_{P_{E}}(\delta g)\right\rangle<x+1$ is bounded by $C_{16}\|g\|^{N_{3}} e^{k_{3} x}$ for some $N_{3}, k_{3}>0$. In fact, according to Lemma 5.1 of [A1], this is true also for $x \leq\left\langle\alpha, H_{P_{E}}(\delta g)\right\rangle$. The claim now follows from (58) taking into account (57).

We now compute the integral of $I(h)$. Let $\{\gamma\}$ be a set of representatives for the double cosets $P_{E} \backslash G / H$ other than the trivial coset $P_{E} H$. We may choose them so that $\gamma \bar{\gamma}^{-1} \in N_{G}\left(T_{E}\right)$. We also assume that $\eta \in\{\gamma\}$ is one of the representatives. Set

$$
E_{\gamma}(h, \varphi, \lambda)=\sum_{\delta \in P_{E} \backslash P_{E} \gamma H} \varphi(\delta h) e^{\left\langle\lambda+\rho_{P}, H_{P_{E}}(\delta h)\right\rangle} .
$$

Then

$$
I(h)=\sum_{\{\gamma\}} E_{\gamma}(h, \varphi, \lambda)
$$

We have shown that the series

$$
\left|E_{\gamma}\right|(h, \varphi, \lambda)=\sum_{\delta \in P_{E} \backslash P_{E} \gamma H}|\varphi(\delta h)| e^{\left\langle\operatorname{Re} \lambda+\rho_{P}, H_{P_{E}}(\delta h)\right\rangle}
$$

are integrable for $\left\langle\operatorname{Re} \lambda, \alpha^{\vee}\right\rangle>>0$. We will show that

$$
\int_{H \backslash H(\mathbb{A})^{1}} E_{\gamma}(h, \varphi, \lambda)=0
$$

for $\gamma \neq \eta$.

As before, we let $P_{\gamma}$ be the subgroup of $H$ such that

$$
P_{\gamma}(F)=P_{E}(E) \cap \gamma H(F) \gamma^{-1}
$$


and let $R=R_{\gamma}$ be the unipotent radical of $P_{\gamma}$. Then we have

$$
\begin{aligned}
& \int_{H \backslash H(\mathbb{A})^{1}} E_{\gamma}(h, \varphi, \lambda) d h=\int_{P_{\gamma}(F) \backslash \gamma H(\mathbb{A})^{1} \gamma^{-1}} \varphi(h \gamma) e^{\left\langle\lambda+\rho_{P}, H_{P_{E}}(h \gamma)\right\rangle} d h \\
& =\int_{P_{\gamma}(F) R(\mathbb{A}) \backslash \gamma H(\mathbb{A})^{1} \gamma^{-1}} e^{\left\langle\lambda+\rho_{P}, H_{P_{E}}(h \gamma)\right\rangle}\left(\int_{R(F) \backslash R(\mathbb{A})} \varphi(u h \gamma) d u\right) d h .
\end{aligned}
$$

We will show that the inner integral vanishes due to the cuspidality of $\varphi$.

Let $w=\gamma \bar{\gamma}^{-1}$ represent an element in $\Omega_{2}$ and set $K=P_{E} \cap w^{-1} P_{E} w$. Define an involution $\Xi$ on $G(E)$ by the formula $\Xi(x)=w^{-1} \bar{x} w$. Then $P_{\gamma}$ is the set of fixed points of $\Xi$ in $P_{E}$. Thus $P_{\gamma} \subset K$ and therefore $P_{\gamma}$ is also equal to the set $K^{\Xi}$ of fixed points of $\Xi$ in $K$. Now $K$ contains four subgroups

$$
\begin{array}{ll}
K_{0}=M_{E} \cap w^{-1} M_{E} w, & K_{2}=N_{E} \cap w^{-1} M_{E} w, \\
K_{1}=M_{E} \cap w^{-1} N_{E} w, & K_{3}=N_{E} \cap w^{-1} N_{E} w .
\end{array}
$$

The subgroups $K_{1}$ and $K_{2}$ normalize $K_{3}$ and the set of commutators $\left[K_{1}, K_{2}\right]$ is contained in $K_{3}$. Hence $U=K_{1} K_{2} K_{3}$ is a unipotent subgroup of $K$ which is normal in $K$ since $K_{0}$ normalizes $K_{1}, K_{2}$, and $K_{3}$. Furthermore, $K=K_{0} \ltimes U$ and $P_{\gamma}=K_{0}^{\Xi} \ltimes U^{\Xi}$. In particular, $R_{\gamma}=U^{\Xi}$.

We claim that for any $u_{1} \in K_{1}$, there exists $u_{3} \in K_{3}$ such that $u_{1} \Xi\left(u_{1}\right) u_{3} \in R$. Indeed, $u_{1} \Xi\left(u_{1}\right) u_{3}$ is fixed by $\Xi$ if and only if

$$
\Xi\left(u_{3}\right) u_{3}^{-1}=u_{1}^{-1} \Xi\left(u_{1}\right)^{-1} u_{1} \Xi\left(u_{1}\right) .
$$

This equation has a solution $u_{3}$ in $K_{3}$ since the right-hand side belongs to $K_{3}$ and satisfies the equation $x \Xi(x)=1$. The coset $u_{3} K_{3}^{\Xi}$ is uniquely determined by $u_{1}$. Set $m\left(u_{1}\right)=\Xi\left(u_{1}\right) u_{3}$. Then the map $u_{1} \longrightarrow u_{1} m\left(u_{1}\right) K_{3}^{\Xi}$ defines a bijection $K_{1} \longrightarrow R / K_{3}^{\Xi}$. This is an isomorphism of algebraic groups whose inverse is the canonical projection onto $M_{E}$. It follows that the formula

$$
\int_{K_{1}(\mathbb{A})}\left(\int_{K_{3}^{\Xi}(\mathbb{A})} f\left(u_{1} m\left(u_{1}\right) t\right) d t\right) d u_{1}
$$

defines an invariant measure on $R(\mathbb{A})$. Here $d u_{1}$ and $d t$ are Haar measures on $K_{1}(\mathbb{A})$ and $K_{3}^{\Xi}(\mathbb{A})$, respectively. If $f$ is left-invariant under $R(F)$,

$$
\int_{R(F) \backslash R(\mathbb{A})} f(r) d r=\int_{K_{1}(F) \backslash K_{1}(\mathbb{A})}\left(\int_{K_{3}^{\Xi}(F) \backslash K_{3}^{\Xi}(A)} f\left(u_{1} m\left(u_{1}\right) t\right) d t\right) d u_{1}
$$

where $d r$ is an invariant measure on $R(F) \backslash R(\mathbb{A})$. If $f$ is a function on $G\left(\mathbb{A}_{E}\right)$ which is left invariant under $N_{E}(\mathbb{A})$, then this formula simplifies to

$$
\int_{R(F) \backslash R(\mathbb{A})} f(r) d r=\int_{K_{1}(F) \backslash K_{1}(\mathbb{A})} f\left(u_{1} m\left(u_{1}\right)\right) d u_{1}
$$

since $K_{3}^{\Xi} \subset N_{E}$ and then to

$$
\int_{R(F) \backslash R(\mathbb{A})} f(r) d r=\int_{K_{1}(F) \backslash K_{1}(\mathbb{A})} f\left(u_{1}\right) d u_{1}
$$

since $u_{1} m\left(u_{1}\right) u_{1}^{-1} \in N_{E}(\mathbb{A})$. 
The function $\varphi$ above is $N_{E}(\mathbb{A})$-invariant, and therefore

$$
\int_{H \backslash H(\mathbb{A})^{1}} E_{\gamma}(h, \varphi, \lambda) d h
$$

is equal to

$$
\int_{P_{\gamma}(F) R(\mathbb{A}) \backslash \gamma H(\mathbb{A})^{1} \gamma^{-1}} e^{\left\langle\lambda+\rho_{P}, H_{P_{E}}(h \gamma)\right\rangle}\left(\int_{K_{1}(F) \backslash K_{1}(\mathbb{A})} \varphi\left(u_{1} h \gamma\right) d u\right) d h .
$$

However $K_{1}$ is the unipotent radical of a (not necessarily standard) parabolic subgroup of $M_{E}$. If $\gamma$ is not admissible (i.e. $\gamma \neq \eta$ according to Lemma 21), then $K_{1} \neq 0$. Since $\varphi$ is cuspidal, the inner integral over $K_{1}$ vanishes as desired. We remark that the idea that these terms vanish already appears in [F3].

We have shown that

$$
\begin{aligned}
\Pi^{G / H}(E(\varphi, \lambda)) & =\int_{H(F) \backslash H(\mathbb{A})^{1}} E_{\eta}(h, \varphi, \lambda) d h \\
& =\int_{H_{\eta}(F) \backslash H(\mathbb{A})^{1}} e^{\left\langle\lambda+\rho_{P}, H_{P_{E}}(\eta h)\right\rangle} \varphi(\eta h) d h .
\end{aligned}
$$

Note that this integral is absolutely convergent. To complete the proof, i.e. to show that $\sigma_{2}^{*}=\overline{\sigma_{1}}$, we rewrite the expression for $\Pi^{G / H}(E(\varphi, \lambda))$ slightly as follows. Let

$$
\xi=\left(\begin{array}{ll}
0 & 1_{m} \\
1_{m} & 0
\end{array}\right)
$$

We may choose $\eta$ to be

$$
\eta=\left(\begin{array}{cc}
1_{m} & i 1_{m} \\
1_{m} & -i 1_{m}
\end{array}\right)
$$

where $i \in E-F$ and $i^{2} \in F$. Then

$$
M_{\eta}=\left\{m=\operatorname{diag}(g, \bar{g}): g \in G L_{m}(E)\right\}
$$

and $H_{\eta}=\eta^{-1} M_{\eta} \eta$. For future reference, we write this as a separate proposition. Henceforth, we assume that $\varphi(a g)=\varphi(g)$ for $a \in A_{P}$.

Proposition 26. With the previous notation, we have, for $\left\langle\operatorname{Re} \lambda, \alpha^{\vee}\right\rangle>>0$,

$$
\begin{gathered}
\Pi^{G / H}(E(\varphi, \lambda))= \\
\int_{H_{\eta}(\mathbb{A}) \backslash H(\mathbb{A})} e^{\left\langle\lambda+\rho_{P}, H_{P_{E}}(\eta h)\right\rangle}\left(\int_{M_{\eta} \backslash M_{\eta}(\mathbb{A})^{1}} \varphi(m \eta h) d m\right) d h,
\end{gathered}
$$

where the integral on the right is absolutely convergent.

Example 1. We illustrate the decomposition of the group $P_{\gamma}$ used above in the case that $P$ corresponds to $(2,1,1,1)$ and $w=(13)(24)$ :

$$
P_{E}=\left\{\left(\begin{array}{ccccc}
\# & \# & * & * & * \\
\# & \# & * & * & * \\
0 & 0 & \# & * & * \\
0 & 0 & 0 & \# & * \\
0 & 0 & 0 & 0 & \#
\end{array}\right)\right\}, w P_{E} w^{-1}=\left\{\left(\begin{array}{ccccc}
\# & * & 0 & 0 & * \\
0 & \# & 0 & 0 & * \\
* & * & \# & \# & * \\
* & * & \# & \# & * \\
0 & 0 & 0 & 0 & \#
\end{array}\right)\right\} .
$$


Then

$$
K=P_{E} \cap w^{-1} P_{E} w=\left\{\left(\begin{array}{lllll}
K_{0} & K_{1} & 0 & 0 & K_{3} \\
0 & K_{0} & 0 & 0 & K_{3} \\
0 & 0 & K_{0} & K_{2} & K_{3} \\
0 & 0 & 0 & K_{0} & K_{3} \\
0 & 0 & 0 & 0 & K_{0}
\end{array}\right)\right\}
$$

where we have indicated which entries belong to the subgroups $K_{j}$. In this case

$$
P_{\gamma}=\left\{\left(\begin{array}{ccccc}
\alpha & \varepsilon & 0 & 0 & \nu \\
0 & \beta & 0 & 0 & \mu \\
0 & 0 & \bar{\alpha} & \bar{\varepsilon} & \bar{\nu} \\
0 & 0 & 0 & \bar{\beta} & \bar{\mu} \\
0 & 0 & 0 & 0 & a
\end{array}\right): \alpha, \beta \in E^{*}, \varepsilon, \mu, \nu \in E \text { and } a \in F^{*}\right\} .
$$

The integral over $\varepsilon$ will lead to vanishing of the integral when $\varphi$ is in the induced space of a space of cusp forms on $M_{P}$.

The proof of Theorem 23 establishes the convergence of the integral in the previous Proposition. However, we will need to establish the convergence of the integral for functions $\varphi$ which are not necessarily cuspidal, in fact, for $\varphi$ a constant function. If $\varphi=1$, then the inner integral is just the volume of the quotient $M_{\eta}(F) \backslash M_{\eta}\left(\mathbb{A}_{F}\right)^{1}$. The remaining integral is described in the next Lemma.

Lemma 27. Let the notations be as in the previous proposition, except that $\lambda$ is real and $\varphi=1$. If $\left\langle\operatorname{Re} \lambda, \alpha^{\vee}\right\rangle>>0$ is sufficiently large, then the following integral is finite:

$$
\int_{H_{\eta}(\mathbb{A}) \backslash H(\mathbb{A})} e^{\left\langle\lambda+\rho_{P}, H_{P_{E}}(\eta h)\right\rangle} d h .
$$

Proof. It will be convenient to change notations and write the function in the integrand as $f(g, s)$ where $s$ is real and $f$ is defined by:

$$
f\left[\left(\begin{array}{cc}
a & x \\
0 & b
\end{array}\right) k, s\right]=\left|\frac{\operatorname{det} a}{\operatorname{det} b}\right|^{s+m / 2} .
$$

Here the matrices $a, b, x, 0$ are $m \times m$ matrices and $k$ is in $\mathbf{K}_{E}$. We have to show that the integral

$$
\int_{H_{\eta}(\mathbb{A}) \backslash H(\mathbb{A})} f(\eta h, s) d h
$$

is finite for $s$ sufficiently large. To that end we use the familiar device of representing $f$ by an integral. Recall the Zeta integral for the trivial representation $1_{m E}$ of $G L\left(m, \mathbb{A}_{E}\right)$ :

$$
\int_{G L\left(m, \mathbb{A}_{E}\right)} \phi(t)|\operatorname{det} t|^{s+\frac{m-1}{2}} d t
$$

where $\phi$ is a Schwartz-Bruhat function on $M\left(m \times m, \mathbb{A}_{E}\right)$. The integral converges for $s$ sufficiently large and is a holomorphic multiple of the Godement-Jacquet $L$-function $L\left(s, 1_{m E}\right)$. In fact, it equals $L\left(s, 1_{m E}\right)$ for a suitable choice of $\phi$. We can write:

$$
f(g, s)=\frac{1}{L\left(2 s+\frac{m+1}{2}, 1_{m E}\right)} \int_{G L\left(m, \mathbb{A}_{E}\right)} \Phi[(0, t) g]|\operatorname{det} t|_{E}^{2 s+m} d t|\operatorname{det} g|_{E}^{s+m / 2},
$$


where $\Phi$ is a suitable Schwartz-Bruhat function on $M\left(2 m \times m, \mathbb{A}_{E}\right)$. We can write this integral as an integral over $M_{\eta}$ :

$$
f(g, s)=\frac{1}{L\left(2 s+\frac{m+1}{2}, 1_{m E}\right)} \int_{M_{\eta}(\mathbb{A})} \Phi\left[\left(0,1_{m}\right) m g\right]|\operatorname{det} m|_{F}^{2 s+m} d m|\operatorname{det} g|_{E}^{s+m / 2} .
$$

After a change of variables we get, for $h \in H(\mathbb{A})$ :

$$
f(\eta h, s)=\frac{1}{L\left(2 s+\frac{m+1}{2}, 1_{m E}\right)} \int_{H_{\eta}(\mathbb{A})} \Phi\left[\left(0,1_{m}\right) \eta h_{\eta} h\right]\left|\operatorname{det}\left(h_{\eta} h\right)\right|_{F}^{2 s+m} d h_{\eta} .
$$

Combining the integral over $H_{\eta}(\mathbb{A})$ with the integral over the quotient $H_{\eta}(\mathbb{A}) \backslash H(\mathbb{A})$ we get for our integral:

$$
\frac{1}{L\left(2 s+\frac{m+1}{2}, 1_{m E}\right)} \int_{H(\mathbb{A})} \Phi\left[\left(0,1_{m}\right) \eta h\right]|\operatorname{det}(h)|_{F}^{2 s+m} d h .
$$

If

$$
h=\left(\begin{array}{ll}
a & b \\
c & d
\end{array}\right)
$$

then

$$
\Phi\left[\left(0,1_{m}\right) \eta h\right]=\Phi(a-i c, b-i d) .
$$

This is a Schwartz-Bruhat function on $M\left(m \times m, \mathbb{A}_{F}\right)$ and thus the integral is now a Zeta integral for the trivial representation $1_{m F}$ of $G L(m, \mathbb{A})$. In particular, it converges for $s$ sufficiently large and the Lemma is proved.

\section{INTERTWINING PERIODS}

Fix a Levi subgroup $M$ of $H$ and a cuspidal representation $\sigma$ of $M_{E}\left(\mathbb{A}_{E}\right)$ trivial on $A_{P_{E}}$. For $\xi \in \Omega_{2}(M, M), \varphi \in \mathcal{A}_{P}(G)_{\sigma}$ and $\lambda \in\left(\mathfrak{A}_{P, \mathbb{C}}^{*}\right)_{\xi}^{-}$, we claim that the following intertwining period attached to $\xi$ is well-defined:

$$
J(\xi, \varphi, \lambda)=\int_{H_{\eta}(\mathbb{A}) \backslash H(\mathbb{A})} e^{\left\langle\lambda+\rho_{P}, H_{P_{E}}(\eta h)\right\rangle}\left(\int_{M_{\eta}(F) \backslash M_{\eta}(\mathbb{A})^{1}} \varphi(m \eta h) d m\right) d h
$$

where $\eta \in G(E)$ is any element satisfying $\eta \bar{\eta}^{-1}=\xi$. Recall that $P_{\eta}=M_{\eta} N_{\eta}$, $H_{\eta}=\eta^{-1} P_{\eta} \eta$ and that the center of $M_{\eta}$ is contained in the center of $M_{E}$. It follows that the inner integral defines a function of $h$ invariant on the left under the subgroup $H_{\eta}(\mathbb{A})$. The function $h \longmapsto e^{\left\langle\lambda, H_{P_{E}}(\eta h)\right\rangle}$ is also left-invariant under $H_{\eta}(\mathbb{A})$, for if $x \in H_{\eta}(\mathbb{A})$, then

$$
\left\langle\lambda, H_{P_{E}}(\eta x h)\right\rangle=\left\langle\lambda, H_{P_{E}}(y \eta h)\right\rangle=\left\langle\lambda, H_{P_{E}}(y)\right\rangle+\left\langle\lambda, H_{P_{E}}(\eta h)\right\rangle
$$

where $y=\eta x \eta^{-1} \in P_{\eta}(\mathbb{A})$. But $\left\langle\lambda, H_{P_{E}}(y)\right\rangle=0$ since $H_{P_{E}}(y)$ lies in the subspace $\mathfrak{A}_{P}^{+}$of $\mathfrak{A}_{P}$ fixed by $\xi$ for all $y \in P_{\eta}(\mathbb{A})$. Moreover, the function $h \longmapsto e^{\left\langle\rho_{P}, H_{P_{E}}(\eta h)\right\rangle}$ is the right modulus of the subgroup $H_{\eta}(\mathbb{A})$ (cf. the proof of Proposition 22) while the group $H(\mathbb{A})$ is unimodular. Finally it depends only on the double coset $P(F) \eta H(F)$ corresponding to $\xi$. We prove below that the integral defining $J(\xi, \varphi, \lambda)$ converges absolutely for $\lambda$ in a suitable cone and can be analytically continued to a meromorphic function of $\lambda \in\left(\mathfrak{A}_{P}^{*}\right)_{\xi}^{-}$. 
Example 2. In the special case where $P=B$ and $\xi=e$, we may take $\eta=e$. Note that $\lambda$ is necessarily 0 . In this case, $H_{\eta}=P, M_{\eta}=M$, and the Iwasawa decomposition yields

$$
J(e, \varphi, \lambda)=\operatorname{vol}\left(M(F) \backslash M(\mathbb{A})^{1}\right) \int_{\mathbf{K}_{H}} \varphi(k) d k
$$

where $\mathbf{K}_{H}$ is a maximal compact subgroup of $H(\mathbb{A})$.

Example 3. Let the notation be as in Theorem 23. Then we find:

$$
\Pi^{G / H}(E(\varphi, \lambda))=\int_{H_{\eta}(F) \backslash H(\mathbb{A})^{1}} e^{\left\langle\lambda+\rho_{P}, H_{P_{E}}(\eta h)\right\rangle} \varphi(\eta h) d h=J(\xi, \varphi, \lambda)
$$

where $H_{\eta}=\eta^{-1} M_{\eta} \eta$ and $M_{\eta}=\left\{\operatorname{diag}(g, \bar{g}): g \in G L_{m}(E)\right\}$.

16. Minimal involutions. To investigate the functionals $J(\xi, \varphi, \lambda)$, we use induction beginning with the minimal involutions, defined as follows.

Definition 2. We say that $\xi \in \Omega_{2}(M, M)$ is minimal if $\left(\mathfrak{A}_{P}^{*}\right)_{\xi}^{-}$is spanned by simple roots in $\Delta_{P}$.

This definition as well as several results below apply to groups other than $G L(n)$. In our case, $P$ corresponds to a partition $\left(n_{1}, \ldots, n_{r}\right)$ of $n$ such that $M \simeq G L\left(n_{1}\right) \times$ $\cdots \times G L\left(n_{r}\right)$. An involution $\xi \in \Omega_{2}(M, M)$ permutes the factors of $M$ and hence induces a permutation of order two on $\{1,2, \ldots, r\}$. It is easy to see that $\xi$ is minimal if and only if the permutation is a product of disjoint transpositions of the form $(j, j+1)$.

Let $Y$ be the set of $j \in\{1,2, \ldots, r-1\}$ such that $n_{j}=n_{j+1}$. For each subset $X \subset Y$ such that $j+1 \notin X$ if $j \in X$, there is a unique minimal involution $\xi$ that interchanges $G L\left(n_{j}\right)$ and $G L\left(n_{j+1}\right)$ for $j \in X$ and fixes the remaining factors. Let $Q$ be the parabolic subgroup containing $P$ that corresponds to the partition obtained from $\left(n_{1}, \ldots, n_{r}\right)$ by replacing the pair of entries $n_{j}, n_{j+1}$ by the single entry $n_{j}+n_{j+1}$ for $j \in X$. In other words, $\Delta_{P}^{Q}$ spans $\left(\mathfrak{A}_{P}^{*}\right)_{\xi}^{-}$. Then $Q$ uniquely determines $X$ and we may denote the minimal involution associated to $X$ by $\xi_{Q}$.

Let $\Xi$ be an associate class of standard Levi subgroups. Since each $M \in \Xi$ lies in a unique standard parabolic subgroup $P$, there is no ambiguity if we use the index $M$ instead of $P$. For example, we shall write $\Delta_{M}$ for $\Delta_{P}$, etc. Let $\Phi_{M}$ be the set of roots of $A_{P}$ and let $\Phi_{M}^{+}$be the subset of positive roots (those that occur in $N_{P}$ where $\left.M=M_{P}\right)$. For $w \in \Omega(M)$ we define the length $\ell_{M}(w)$ to be the number of roots in $\Phi_{M}^{+}$sent to negative roots in $\Phi_{w M w^{-1}}$ by $w$. For all $\alpha \in \Delta_{M}$, there is an elementary symmetry $s_{\alpha} \in \Omega(M)$ uniquely characterized by the property that $\alpha$ is the only positive root sent to a negative root by $s_{\alpha}$ (cf. [MW], Section 1.1.7). Furthermore, for $w \in \Omega(M)$ and $\alpha \in \Delta_{w M w^{-1}}$, we have

$$
\ell_{\mathrm{M}}\left(s_{\alpha} w\right)=\left\{\begin{array}{l}
\ell_{M}(w)+1 \text { if } w^{-1} \alpha>0 \\
\ell_{M}(w)-1 \text { if } w^{-1} \alpha<0 .
\end{array}\right.
$$

17. Graph of involutions. We will consider a directed graph $\Gamma$ whose vertices are elements $(\xi, M)$ where $M \in \Xi$ and $\xi \in \Omega_{2}(M, M)$. To define the edges, observe that for all $\alpha \in \Delta_{M}$, the pair $\left(s_{\alpha} \xi s_{\alpha}^{-1}, s_{\alpha} M s_{\alpha}^{-1}\right)$ is also a vertex of $\Gamma$. We define an edge

$$
(\xi, M) \stackrel{\alpha}{\longrightarrow}\left(s_{\alpha} \xi s_{\alpha}^{-1}, s_{\alpha} M s_{\alpha}^{-1}\right)
$$


provided that $\xi(\alpha) \neq \alpha$. Note that an edge may start and end at the same vertex. By Lemma 28 below, this occurs if and only if $\xi(\alpha)=-\alpha$. Furthermore, if the edge (61) has distinct vertices, then there is also an edge in the opposite direction:

$$
(\xi, M) \stackrel{-s_{\alpha} \alpha}{\longleftarrow}\left(s_{\alpha} \xi s_{\alpha}^{-1}, s_{\alpha} M s_{\alpha}^{-1}\right) .
$$

Let $\Gamma^{0}$ be the subgraph whose vertices are the same at $\Gamma$, but containing those edges (61) for which $\xi(\alpha) \neq \pm \alpha$. In other words, $\Gamma^{0}$ is obtained from $\Gamma$ by removing the loops.

Example 4. Consider the case $G=G L(3), \Xi=\{M\}$ the class of the Levi subgroup of the Borel subgroup. Set $\xi_{1}=(12), \xi_{2}=(23), \xi_{3}=(13)$. Then $\Gamma$ is the following graph with two connected components:

$$
\stackrel{\alpha_{2}}{\circlearrowright}\left(\xi_{2}, M\right) \stackrel{\alpha_{1}}{\longrightarrow}\left(\xi_{3}, M\right) \stackrel{\alpha_{2}}{\longrightarrow}\left(\xi_{1}, M\right) \stackrel{\alpha_{1}}{\circlearrowleft} \quad(e, M) .
$$

Here we have not drawn the two interior edges directed from right to left. The graph $\Gamma^{0}$ is obtained by removing the loops.

Example 5. Consider the case $G=G L(4), \Xi=\{M\}$ the class of the Levi subgroup of the Borel subgroup. Set $\xi_{1}=(12)(34), \xi_{2}=(13)(24), \xi_{3}=(14)(23)$. Then $\Gamma$ contains the component

$$
\underset{\substack{\alpha_{3} \\ \circlearrowright}}{\stackrel{\alpha_{1}}{\alpha_{3}}}\left(\xi_{1}, M\right) \stackrel{\alpha_{2}}{\longrightarrow}\left(\xi_{2}, M\right) \underset{\stackrel{\alpha_{3}}{\longrightarrow}}{\stackrel{\alpha_{1}}{\longrightarrow}}\left(\xi_{3}, M\right) \stackrel{\alpha_{2}}{\circlearrowleft} .
$$

Again, we have not drawn the interior edges directed from right to left.

Each path

$$
\left(\xi_{1}, M_{1}\right) \stackrel{\alpha_{1}}{\longrightarrow}\left(\xi_{2}, M_{2}\right) \stackrel{\alpha_{2}}{\longrightarrow} \ldots \stackrel{\alpha_{l-1}}{\longrightarrow}\left(\xi_{\ell}, M_{\ell}\right)
$$

in $\Gamma$ defines a word $s=s_{\alpha_{\ell-1}} \cdots s_{\alpha_{1}} \in \Omega\left(M_{1}, M_{l}\right)$ such that $\xi_{\ell}=s \xi_{1} s^{-1}$ and $M_{\ell}=s M_{1} s^{-1}$. If $M_{1}$ and $M_{2}$ are standard Levi subgroups and $\xi_{i} \in \Omega_{2}\left(M_{i}, M_{i}\right)$ for $i=1,2$, let $\Omega\left(\xi_{1}, \xi_{2}\right)$ be the set of all words defined by paths from $\left(\xi_{1}, M_{1}\right)$ to $\left(\xi_{2}, M_{2}\right)$ in $\Gamma$. Let $\Omega^{0}\left(\xi_{1}, \xi_{2}\right)$ be defined similarly relative to the graph $\Gamma^{0}$.

Lemma 28. Assume $\xi \in \Omega_{2}(M, M)$ and $\alpha \in \Delta_{M}$. Then

(1) The following are equivalent:

(i) $s_{\alpha} \xi s_{\alpha}^{-1}=\xi$;

(ii) $\xi(\alpha)= \pm \alpha$;

(iii) $\ell_{s_{\alpha} M s_{\alpha}^{-1}}\left(s_{\alpha} \xi s_{\alpha}^{-1}\right)=\ell_{M}(\xi)$.

Furthermore, if $\xi(\alpha)= \pm \alpha$, then $s_{\alpha} M s_{\alpha}^{-1}=M$.

(2) If $\xi \alpha \neq \pm \alpha$, then the following are equivalent:

(i) $\ell_{s_{\alpha} M s_{\alpha}^{-1}}\left(s_{\alpha} \xi s_{\alpha}^{-1}\right)=\ell_{M}(\xi)-2$;

(ii) $\xi(\alpha)<0$.

Proof. We first prove (1). Since $G=G L(n), M$ is isomorphic to a product $G L\left(n_{1}\right) \times$ $\cdots \times G L\left(n_{r}\right)$ where $\left(n_{1}, \ldots, n_{r}\right)$ is a partition of $n$. The elementary reflection $s_{\alpha}$ interchanges two adjacent factors $G L\left(n_{k}\right)$ and $G L\left(n_{k+1}\right)$ for some $k$, and $\xi$ interchanges certain pairs of factors of equal size. Therefore $s_{\alpha} \xi s_{\alpha}^{-1}=\xi$ if and only if either $\xi$ fixes the factors $G L\left(n_{k}\right)$ and $G L\left(n_{k+1}\right)$ (in which case $\xi \alpha=\alpha$ ) or interchanges them (in which case $\xi \alpha=-\alpha$ ). Therefore (i) and (ii) are equivalent. By (60), (iii) occurs if and only if either $\xi(\alpha)>0$ and $s_{\alpha} \xi(\alpha)<0$, or $\xi(\alpha)<0$ and $s_{\alpha} \xi(\alpha)>0$. But this is the case precisely when $\xi(\alpha)= \pm \alpha$. Therefore (ii) and (iii) 
are equivalent. To prove part (2), observe that $\ell_{s_{\alpha} M s_{\alpha}^{-1}}\left(s_{\alpha} \xi s_{\alpha}^{-1}\right)=\ell_{M}(\xi)-2$ if and only if $\xi^{-1} \alpha$ and $s_{\alpha} \xi^{-1} \alpha$ are both negative, but if $\xi \alpha \neq \pm \alpha$, this is equivalent to the condition $\xi^{-1} \alpha<0$. We remark that (1)(ii),(iii) and (2)(i),(ii) are equivalent for general groups but (1)(i) is sharper than (1)(ii) in general (unless $P=P_{0}$ ).

This lemma implies, in particular, that an edge begins and ends at $(\xi, M)$ if and only if $\xi(\alpha)=-\alpha$. Therefore $\Gamma^{0}$ is the graph obtained from $\Gamma$ by removing the loops.

We shall use the following characterization of minimal involutions.

Proposition 29. Let $\xi \in \Omega_{2}(M, M)$. Then

(1) $\xi$ is minimal if and only if the length function $\ell$ has a local minimum at $\xi$ (i.e., $\ell_{M}(\xi) \leq \ell_{M^{\prime}}\left(\xi^{\prime}\right)$ for all neighbors $\left(\xi^{\prime}, M^{\prime}\right)$ of $(\xi, M)$ in $\left.\Gamma\right)$.

(2) $\xi$ is conjugate to a minimal involution by an element in $\Omega(M)$.

(3) $\left(\mathfrak{A}_{P}^{*}\right)_{\xi}^{-}$is spanned by roots in $\Phi_{P}$.

Proof. Suppose that $\xi$ is a local minimum for $\ell$ on $\Gamma$. Lemma 28 implies that $\xi(\alpha)=-\alpha$ whenever $\alpha \in \Delta_{M}$ and $\xi(\alpha)<0$. Let $I=\left\{\alpha \in \Delta_{M}: \xi(\alpha)=-\alpha\right\}$. If $\beta \in \Phi_{M}^{+}$is any root which is not in the span of the roots in $I$, then $\xi(\beta)>0$ because $\xi(\beta)$ contains positive multiples of simple roots outside $I$. Therefore $\left(\mathfrak{A}_{M}^{*}\right)_{\xi}^{-}$ is contained in the span of $I$ and hence coincides with the span of $I$. Therefore $\xi$ is a minimal involution in $\Omega_{2}(M, M)$.

On the other hand, if $\xi$ is minimal in $\Omega_{2}(M, M)$, then for any $0<\beta \notin\left(\mathfrak{A}_{M}^{*}\right)_{\xi}^{-}$we have $\xi(\beta)>0$. Indeed, we may decompose $\beta$ as $\beta_{\xi}^{+}+\beta_{\xi}^{-}$where $\xi \beta_{\xi}^{ \pm}= \pm \beta_{\xi}^{ \pm}$. Since $\beta_{\xi}^{+} \neq 0$, it contains in its decomposition relative to $\Delta_{M}$ some positive multiple of a simple root $\alpha$ such that $\xi \alpha>0$, and this multiple is also present in the decomposition of $\xi(\beta)=\beta_{\xi}^{+}-\beta_{\xi}^{-}$. In particular, if $\beta \in \Delta_{M}$ and $\xi(\beta)<0$, then $\xi \beta=-\beta$. It follows from Lemma 28 that $\ell$ has a local minimum at $\xi$.

Part (2) follows immediately from (1) by the description of the edges in $\Gamma$. Part (3) follows from (2).

Part (1) of the previous proposition implies

Corollary 30. Let $\xi \in \Omega_{2}(M, M)$. If $(\xi, M)$ is not minimal, then there exists $\alpha \in \Delta_{M}$ such that the element $\xi^{\prime}=s_{\alpha} \xi s_{\alpha}^{-1}$ satisfies $\ell\left(\xi^{\prime}\right)=\ell(\xi)-2$.

18. The functional equations. Fix a parabolic subgroup $P=M N$ and $\xi \in$ $\Omega_{2}(M, M)$. Let $\Phi_{P}^{+}$be the set of positive roots of $A_{P}$ in $N_{P}$ and set

$$
\Phi_{P}^{\xi}=\left\{\beta \in \Phi_{P}^{+}: \xi \beta<0\right\} .
$$

We shall set

$$
D_{P, \xi}=\left\{\lambda \in\left(\mathfrak{A}_{P}^{*}\right)_{\xi}^{-}:\left\langle\lambda, \beta^{\vee}\right\rangle>>0 \text { for } \beta \in \Phi_{P}^{\xi}\right\}
$$

where $>>$ denotes sufficiently large, but we shall not specify the implied constants. However, observe that $D_{P, \xi}$ is non-empty because it contains $P_{\xi}^{-} \lambda$ if $\lambda$ is sufficiently positive in $\mathfrak{A}_{P}^{*+}$. Indeed, if $\beta \in \Phi_{P}^{+}$, then $P_{\xi}^{-} \beta^{\vee}=\frac{1}{2}\left(\beta^{\vee}-\xi \beta^{\vee}\right)$ is a sum of positive roots and hence

$$
\left\langle P_{\xi}^{-} \lambda, \beta^{\vee}\right\rangle=\left\langle\lambda, P_{\xi}^{-} \beta^{\vee}\right\rangle>>0 .
$$


Our main result in this section is the following

Theorem 31. Let $\xi \in \Omega_{2}(M, M)$. Then

(1) $J(\xi, \varphi, \lambda)$ is defined by an absolutely convergent integral for $\operatorname{Re} \lambda \in D_{P, \xi}$.

(2) $J(\xi, \varphi, \lambda)$ has a meromorphic continuation to $\left(\mathfrak{A}_{P}^{*}\right)_{\xi}^{-} \otimes \mathbb{C}$.

(3) If $s \in \Omega\left(\xi_{1}, \xi_{2}\right)$, then

$$
J\left(\xi_{1}, \varphi, \lambda\right)=J\left(\xi_{2}, M(s, \lambda) \varphi, s \lambda\right)
$$

In the case of minimal involutions, parts (1) and (2) of this Theorem as well as certain cases of (3) follow from the previous section. Indeed,

Lemma 32. If $\xi=\xi_{Q}$ is minimal and $Q=Q_{\xi}$, then $\left(\mathfrak{A}_{P}^{*}\right)_{\xi}^{-}=\left(\mathfrak{A}_{P}^{Q}\right)^{*}$ and $D_{P, \xi}$ is the half-space

$$
D_{P, \xi}=\left\{\lambda \in\left(\mathfrak{A}_{P}^{Q}\right)^{*}:\left\langle\operatorname{Re} \lambda, \alpha^{\vee}\right\rangle>>0 \text { for all } \alpha \in \Delta_{P}^{Q}\right\} .
$$

In this case,

(1) The integral defining $J\left(\xi_{Q}, \varphi, \lambda\right)$ is absolutely convergent if $\operatorname{Re} \lambda \in D_{P, \xi}$.

(2) $J\left(\xi_{Q}, \varphi, \lambda\right)$ has a meromorphic continuation to $\left(\mathfrak{A}_{P}^{Q}\right)^{*} \otimes \mathbb{C}$.

(3) $J\left(\xi_{Q}, \varphi, \lambda\right)=J\left(\xi_{Q}, M\left(s_{\alpha}, \lambda\right) \varphi, s_{\alpha} \lambda\right)$ for all $\alpha \in \Delta_{P}^{Q}$.

Proof. We can take $\eta=\eta_{Q} \in M_{Q}(E)$. Then $H(\mathbb{A})=N_{Q}(\mathbb{A}) M_{Q}(\mathbb{A}) \mathbf{K}_{H}$ with $d h=$ $e^{-\left\langle\rho_{Q}, H_{E}(m)\right\rangle} d n d m d k$ and $H_{\eta}=H_{\eta, Q} N_{Q}$ where $H_{\eta, Q}=H_{\eta} \cap M_{Q}$. Therefore, since $\rho_{P}=\rho_{Q}+\rho_{P}^{Q}$

$$
\begin{gathered}
J\left(\xi_{Q}, \phi, \lambda\right) \\
=\int_{H_{n, Q}(\mathbb{A}) N_{Q}(\mathbb{A}) \backslash H(\mathbb{A})} e^{\left\langle\lambda+\rho_{P}, H_{P_{E}}\left(\eta_{Q} h\right)\right\rangle}\left(\int_{M_{\eta}(F) \backslash M_{\eta}(\mathbb{A})^{1}} \phi\left(l \eta_{Q} h\right) d l\right) d h \\
=\int_{H_{n, Q}(\mathbb{A}) \backslash M_{Q}(\mathbb{A})} e^{\left\langle\lambda+\rho_{P}^{Q}, H_{P_{E}}\left(\eta_{Q} m\right)\right\rangle}\left(\int_{M_{\eta}(F) \backslash M_{\eta}(\mathbb{A})^{1}} \phi^{\left.\mathbf{K}_{F}\left(l \eta_{Q} h\right) d l\right)} d m\right. \\
=J^{M_{Q}\left(\xi_{Q}, \phi^{\mathbf{K}_{F}}, \lambda_{P}^{Q}\right),}
\end{gathered}
$$

where we set

$$
\phi^{\mathbf{K}_{F}}(g)=\int_{\mathbf{K}_{F}} \phi(g k) d k .
$$

By Proposition 26 (applied to a product of linear groups, namely $M_{Q_{E}}$ ) this integral is absolutely convergent in the indicated domain and equal to the period $\Pi^{M_{Q_{E}} / M_{Q}}\left(E\left(\phi^{\mathbf{K}_{H}}, \lambda_{P}^{Q}\right)\right)$ of the Eisenstein series on $M_{Q_{E}}$ for the parabolic subgroup $P \cap M_{Q_{E}}$. This shows that the integral extends to a meromorphic function. The stated functional equation is inherited from the functional equation of the Eisenstein series.

The next proposition provides functional equations that can be verified by direct calculation. 
Proposition 33. Let $\xi \in \Omega_{2}(M, M)$ and let $\alpha \in \Delta_{M}$ be a root such that $\xi(\alpha)<0$ but $\xi(\alpha) \neq-\alpha$. Then for $\lambda \in D_{P, \xi}$ we have an equality

$$
J(\xi, \varphi, \lambda)=J\left(s_{\alpha} \xi s_{\alpha}^{-1}, M\left(s_{\alpha}, \lambda\right) \varphi, s_{\alpha} \lambda\right)
$$

where both sides are defined by absolutely convergent integrals.

Proof. We first establish the integral formula (66) below. Let $w_{\alpha}$ be a representative for $s_{\alpha}$ and set:

$$
\xi^{\prime}=w_{\alpha} \xi w_{\alpha}^{-1}, \quad \eta^{\prime}=w_{\alpha} \eta, \quad M^{\prime}=w_{\alpha} M w_{\alpha}^{-1} .
$$

Then $M^{\prime}$ is the standard Levi-subgroup of a (standard) parabolic subgroup $P^{\prime}=$ $M^{\prime} N^{\prime}$. We have $s_{\alpha} \alpha=-\alpha^{\prime}$ with $\alpha^{\prime} \in \Delta_{P^{\prime}}$. We also introduce the parabolic subgroup $R$ such that $R \supset P$ and $\Delta_{P}^{R}=\{\alpha\}$. Note that $R \supset P^{\prime}$ and $\Delta_{P^{\prime}}^{R}=\left\{\alpha^{\prime}\right\}$. The standard Levi-decomposition of $R$ reads $R=M_{R} N_{R}$ and $w_{\alpha}$ belongs to $M_{R}$ and hence normalizes $N_{R}$. On the other hand, we have $N=N_{\alpha} N_{R}$ and $N^{\prime}=$ $N_{\alpha^{\prime}} N_{R}$. Here we denote by $N_{\alpha}$ the abelian subgroup exponential of the eigenspace of the Lie algebra corresponding to $\alpha$. Likewise for $N_{\alpha^{\prime}}$.

Define an involution $\theta$ by $\theta(x)=\left(\xi^{\prime}\right)^{-1} \bar{x}^{\prime} \xi^{\prime}$. The subgroup $N_{\eta^{\prime}}^{\prime}$ is the set of fixed points of $\theta$ in $N^{\prime}$. We claim that

$$
w_{\alpha} N_{\eta} w_{\alpha}^{-1}=N_{\eta^{\prime}}^{\prime} \cap N_{R} .
$$

To prove this, observe first that $N_{\eta}$ is contained in $N_{R}$. Indeed, $N_{\eta}$ is the space of $u \in N$ such that $\xi^{-1} u \xi=\bar{u}$ and therefore is contained in $N \cap \xi^{-1} N \xi$. However, $N \cap \xi^{-1} N \xi$ is contained in $N_{R}$, for if not, then $N_{\alpha}$ would intersect $N \cap \xi^{-1} N \xi$ non-trivially and then $\xi N_{\alpha} \xi^{-1} \subset N$, contradicting our assumption that $\xi(\alpha)<0$. Since $w_{\alpha}$ normalizes $N_{R}$, we also conclude that $w_{\alpha} N_{\eta} w_{\alpha}^{-1}$ is contained in $N_{R}$. If an element $x$ satisfies $\xi^{-1} x \xi=\bar{x}$, then the element $x^{\prime}=w_{\alpha} x w_{\alpha}^{-1}$ is fixed by $\theta$. Therefore $w_{\alpha} N_{\eta} w_{\alpha}^{-1}$ is contained in $N_{R} \cap N_{\eta^{\prime}}^{\prime}$. Similarly we find that $w_{\alpha}^{-1}\left(N_{R} \cap N_{\eta^{\prime}}^{\prime}\right) w_{\alpha}$ is contained in $N_{\eta}$ and the equality (64) follows.

The involution $\theta$ maps $N_{\alpha^{\prime}}$ to $N_{R}$. Indeed, if $u \in N_{\alpha^{\prime}}$, then

$$
\theta(u)=w_{\alpha} \xi^{-1} w_{\alpha}^{-1} \bar{u} w_{\alpha} \xi w_{\alpha}^{-1} .
$$

The element $w_{\alpha}^{-1} \bar{u} w_{\alpha}$ is in $N_{-\alpha}$ and thus $\xi^{-1} w_{\alpha}^{-1} \bar{u} w_{\alpha} \xi$ lies in some $N_{\beta}$ with $\beta>0$, $\beta \neq \alpha$. It follows that $\beta^{\prime}=s_{\alpha} \beta$ is a positive root in $\Delta_{P^{\prime}}$ different from $\alpha^{\prime}$ and hence $\theta(u)$ lies in $N_{\beta^{\prime}} \subset N_{R}$.

We now claim that given any element $u \in N_{\alpha^{\prime}}$, there exists $s(u) \in N_{R}$, unique modulo $N_{\eta^{\prime}}^{\prime} \cap N_{R}$, such that $u \theta(u) s(u) \in N_{\eta^{\prime}}^{\prime}$. Furthermore, the map

$$
\begin{aligned}
N_{\alpha^{\prime}} & \longrightarrow N_{\eta^{\prime}}^{\prime} / N_{\eta^{\prime}}^{\prime} \cap N_{R} \\
u & \mapsto u \theta(u) s(u) \quad\left(N_{\eta^{\prime}}^{\prime} \cap N_{R}\right)
\end{aligned}
$$

defines an algebraic group isomorphism (locally, globally), whose inverse is the projection on the $M_{R}$ part. To prove the claim, observe that the element $t=$ $u^{-1} \theta(u)^{-1} u \theta(u)$ belongs to $N_{R}$ and satisfies $\theta(t) t=1$. Hence $t=\theta(s) s^{-1}$ for some $s \in N_{R}$ which is unique modulo $N_{\eta^{\prime}}^{\prime} \cap N_{R}$. Since $u \theta(u) s$ is fixed by $\theta$, we may set $s(u)=s$. 
The isomorphism (65) can be used to compute the Haar measure on $N_{\eta^{\prime}}^{\prime}$ (locally or globally) as:

$$
\int_{N_{\eta^{\prime}}^{\prime}} f(z) d z=\int_{N_{\alpha^{\prime}}}\left(\int_{N_{\eta^{\prime}}^{\prime} \cap N_{R}} f(u \theta(u) s(u) n) d n\right) d u .
$$

To continue the proof, we start with the intertwining period

$$
J\left(s_{\alpha} \xi s_{\alpha}^{-1}, M\left(s_{\alpha}, \lambda\right) \phi, s_{\alpha} \lambda\right)
$$

which is equal to

$$
\int_{H_{\eta^{\prime}}(\mathbb{A}) \backslash H(\mathbb{A})} \int_{M_{\eta^{\prime}}(F) \backslash M_{\eta^{\prime}}(\mathbb{A})^{1}} \int_{N_{\alpha^{\prime}}(\mathbb{A})} e^{\left\langle\lambda+\rho_{P}, H_{P_{E}}\left(w_{\alpha}^{-1} n m^{\prime} \eta^{\prime} h\right)\right\rangle} \phi\left(w_{\alpha}^{-1} n m^{\prime} \eta^{\prime} h\right) d n d m^{\prime} d h .
$$

We will check below that this triple integral is absolutely convergent. The integrand, viewed as a function of $n \in N^{\prime}(\mathbb{A})$, is left-invariant under $N_{R}(\mathbb{A})$. It follows that the integral over $N_{\alpha^{\prime}}(\mathbb{A})$ can be rewritten as an integral over the quotient

$$
w_{\alpha} N_{\eta} w_{\alpha}^{-1}(\mathbb{A}) \backslash N_{\eta^{\prime}}^{\prime}(\mathbb{A}) .
$$

Now we have $P_{\eta^{\prime}}=M_{\eta^{\prime}} N_{\eta^{\prime}}^{\prime}$ and $M_{\eta^{\prime}}=w_{\alpha} M_{\eta} w_{\alpha}^{-1}$. Also $N_{\eta^{\prime}}^{\prime} \supset w_{\alpha} N_{\eta} w_{\alpha}^{-1}$. Thus $P_{\eta^{\prime}}=w_{\alpha} P_{\eta} w_{\alpha}^{-1} N_{\eta^{\prime}}^{\prime}$ with $N_{\eta^{\prime}}^{\prime}$ normal and the intersection of the two subgroups is $w_{\alpha} N_{\eta} w_{\alpha}^{-1}$. Conjugating by $\left(\eta^{\prime}\right)^{-1}$, we obtain

$$
H_{\eta^{\prime}}=H_{\eta}\left(\eta^{\prime-1} N_{\eta^{\prime}}^{\prime} \eta^{\prime}\right) \text {. }
$$

The second group is normal and the intersection of the two groups is $\eta^{-1} N_{\eta} \eta$. Using the fact that $m^{\prime}$ normalizes both $N_{\eta^{\prime}}^{\prime}(\mathbb{A})$ and $w_{\alpha} N_{\eta} w_{\alpha}^{-1}(\mathbb{A})$ and conjugation by $m^{\prime}$ does not change the Haar measures, we see that

$$
J\left(s_{\alpha} \xi s_{\alpha}^{-1}, M\left(s_{\alpha}, \lambda\right) \phi, s_{\alpha} \lambda\right)
$$

can be written as

$$
\iiint e^{\left\langle\lambda+\rho, H_{P_{E}}\left(w_{\alpha}^{-1} m^{\prime} \eta^{\prime} u h\right)\right\rangle} \phi\left(w_{\alpha}^{-1} m^{\prime} \eta^{\prime} u h\right) d m^{\prime} d u d h .
$$

The integral in the $h$ variable is taken over $H_{\eta^{\prime}}(\mathbb{A}) \backslash H(\mathbb{A})$ and the integral in the $u$ variable is taken over $\eta^{-1} N_{\eta} \eta(\mathbb{A}) \backslash\left(\eta^{\prime}\right)^{-1} N_{\eta^{\prime}}^{\prime} \eta^{\prime}(\mathbb{A})$. We may now combine the integrations over $u$ and $h$ into an integration over $H(\mathbb{A}) \operatorname{modulo} H_{\eta}(\mathbb{A})$ to obtain

$$
\int_{H_{\eta}(\mathbb{A}) \backslash H(\mathbb{A})} \int_{M_{\eta^{\prime}}(F) \backslash M_{\eta^{\prime}}(\mathbb{A})^{1}} e^{\left\langle\lambda+\rho_{P}, H_{P_{E}}\left(w_{\alpha}^{-1} m^{\prime} \eta^{\prime} h\right)\right\rangle} \phi\left(w_{\alpha}^{-1} m^{\prime} \eta^{\prime} h\right) d m^{\prime} d h .
$$

Finally, using that $w_{\alpha}^{-1} m^{\prime} \eta^{\prime}=m \eta$ with $m \in M_{\eta}$, we obtain equation (63) as desired.

It remains to verify that the triple integral defining $J\left(\xi^{\prime}, M\left(s_{\alpha}, \lambda\right) \phi, s_{\alpha} \lambda\right)$ is absolutely convergent. To this end, we take the variable $\lambda$ to be real and $\phi$ to be the constant 1 , and define a "scalar" intertwining period:

$$
j(\xi, \lambda)=\operatorname{vol}\left(M_{\eta}(F) \backslash M_{\eta}^{1}(\mathbb{A})\right) \int_{H_{\eta}(\mathbb{A}) \backslash H(\mathbb{A})} e^{\left\langle\lambda+\rho_{P}, H_{P_{E}}(\eta h)\right\rangle} d h
$$

(with positive integrand). Computing formally as above, we find a formula:

$$
j(\xi, \phi)=j\left(s_{\alpha} \xi s_{\alpha}^{-1}, s_{\alpha} \lambda\right) m\left(s_{\alpha}, \lambda\right)
$$


where

$$
m\left(s_{\alpha}, \lambda\right)=\int_{N_{\alpha^{\prime}}(\mathbb{A})} e^{\left\langle\lambda+\rho_{P}, H_{P_{E}}\left(w_{\alpha}^{-1} n\right)\right\rangle} d n
$$

is a scalar intertwining operator. The integral defining $m\left(s_{\alpha}, \lambda\right)$ is known to converge absolutely in a domain

$$
C=\left\{\lambda:\left\langle\lambda, \alpha^{\vee}\right\rangle>>0\right\} .
$$

By Lemma 27 above, we know that if $\xi$ is minimal, then $j(\xi, \lambda)$ converges in some domain $D_{P, \xi}$. By induction on the length of $\xi$ we may assume that $j\left(\xi^{\prime}, \lambda^{\prime}\right)$ converges in $D_{P^{\prime}, \xi^{\prime}}$. Now

$$
D_{P, \xi} \subset s_{\alpha}^{-1} D_{P^{\prime}, \xi} \cap C .
$$

Thus for $\lambda \in D_{P, \xi}$ we find that both $j\left(s_{\alpha} \xi s_{\alpha}^{-1}, s_{\alpha} \lambda\right)$ and $m\left(s_{\alpha}, \lambda\right)$ are defined by absolutely convergent integrals and our assertion follows: the integral $j(\xi, \lambda)$ is absolutely convergent for $\lambda \in D_{P, \xi}$. The same is therefore true of $J(\xi, \phi, \lambda)$, and the proof is complete.

We now prove parts (1) and (2) of Theorem 31 by induction on $\ell_{M}(\xi)$. If $\xi$ is not minimal, there exists a root $\alpha \in \Delta_{M}$ such that $\xi(\alpha)<0$ and $\xi^{\prime}=s_{\alpha} \xi s_{\alpha}^{-1}$ has length $\ell(\xi)-2$ by Corollary 30 . Let $P^{\prime}$ be the parabolic subgroup with $\mathfrak{A}_{P^{\prime}}^{*}=s_{\alpha} A_{P}^{*}$. Then

$$
D_{P, \xi}=s_{\alpha}^{-1}\left(D_{P^{\prime}, \xi^{\prime}}\right) \cap\left\{\lambda \in\left(\mathfrak{A}_{P}^{*}\right)_{\xi}^{-}:\left\langle\lambda, \alpha^{\vee}\right\rangle,\left\langle\lambda,-\xi \alpha^{\vee}\right\rangle>>0\right\}
$$

since $\Phi_{P}^{\xi}=s_{\alpha} \Phi_{P^{\prime}}^{\xi^{\prime}} \cup\{\alpha,-\xi(\alpha)\}$. We may assume by induction that the integral defining $J\left(\xi^{\prime}, \varphi, \lambda\right)$ converges absolutely in $D_{\xi^{\prime}}$ and has a meromorphic continuation. Then $J(\xi, \varphi, \lambda)$ converges absolutely in $D_{\xi}$ and has a meromorphic continuation by Proposition 33 .

It remains to prove part (3) of Theorem 31. The functional equations corresponding to $s \in \Omega^{0}\left(\xi, \xi^{\prime}\right)$ are a consequence of Proposition 33 and the functional equations $M\left(s_{\alpha}^{-1}, s_{\alpha} \lambda\right) M\left(s_{\alpha}, \lambda\right)=I$. It remains to examine the case of a loop. So assume that $\xi=s_{\alpha} \xi s_{\alpha^{-1}}$ where $\alpha$ is a root such that $\xi \alpha=-\alpha$. By Lemma 29, (2), there exists a minimal involution $\xi^{\prime}$ such that $\xi^{\prime}=w \xi w^{-1}$ with $w \in \Omega^{0}\left(\xi, \xi^{\prime}\right)$, i.e., $w$ is equal to a word associated to a path in $\Gamma^{0}$ (start with any $w \in \Omega\left(\xi, \xi^{\prime}\right)$ and remove loops in the path attached to $w$ ). Since $s_{\alpha} \xi s_{\alpha}^{-1}=\xi$, the element $s=w s_{\alpha} w^{-1}$ lies in $\Omega\left(\xi^{\prime}, \xi^{\prime}\right)$. In fact, since $s$ acts trivially on $\left(\mathfrak{A}_{P^{\prime}}^{*}\right)_{\xi^{\prime}}^{+}$and $\xi^{\prime}$ is minimal, $s$ must be a product of commuting elementary symmetries $s_{\gamma}$ for roots $\gamma$ contained in $\left(\mathfrak{A}_{P^{\prime}}^{*}\right)_{\xi^{\prime}}^{-}$. We deduce that

$$
\begin{aligned}
J\left(\xi, M\left(s_{\alpha}, \lambda\right) \varphi, s_{\alpha} \lambda\right) & =J\left(\xi^{\prime}, M\left(w, s_{\alpha} \lambda\right) M\left(s_{\alpha}, \lambda\right) \varphi, w s_{\alpha} \lambda\right) \\
& =J\left(\xi^{\prime}, M\left(w s_{\alpha}, \lambda\right) \varphi, w s_{\alpha} \lambda\right)
\end{aligned}
$$

by the functional equation $M\left(w s_{\alpha}, \lambda\right)=M\left(w, s_{\alpha} \lambda\right) M\left(s_{\alpha}, \lambda\right)$. On the other hand, by Lemma 32

$$
\begin{aligned}
J\left(\xi^{\prime}, M\left(w s_{\alpha}, \lambda\right) \varphi, w s_{\alpha} \lambda\right) & =J\left(\xi^{\prime}, M\left(s w s_{\alpha}, \lambda\right) \varphi, s w s_{\alpha} \lambda\right) \\
& =J\left(\xi^{\prime}, M(w, \lambda) \varphi, w \lambda\right) .
\end{aligned}
$$

Finally $J\left(\xi^{\prime}, M(w, \lambda) \varphi, w \lambda\right)=J(\xi, \varphi, \lambda)$, again since $w \in \Omega^{0}\left(\xi, \xi^{\prime}\right)$. 
19. A description of $\Omega\left(\xi, \xi^{\prime}\right)$. Since the sets $\Omega\left(\xi, \xi^{\prime}\right)$ determine the functional equations of the intertwining periods, it is of interest to have a description of them independent of the graphs. We do this in the next proposition. For $\xi \in \Omega_{2}(M, M)$, set

$$
C_{\xi}=\left\{x \in \mathfrak{A}_{M}^{*}:\left\langle x, \beta^{\vee}\right\rangle>0 \text { for } \beta \in \Phi_{M}^{+} \cap\left(\mathfrak{A}_{M}^{*}\right)_{\xi}^{+}\right\} .
$$

Proposition 34. Let $M$ and $M^{\prime}$ be standard Levi subgroups, $\xi \in \Omega_{2}(M, M)$, and $\xi^{\prime} \in \Omega_{2}\left(M^{\prime}, M^{\prime}\right)$. Suppose that $w \xi w^{-1}=\xi^{\prime}$. Then the following are equivalent:

(a) $w \in \Omega\left(\xi, \xi^{\prime}\right)$.

(b) $w \in \Omega\left(M, M^{\prime}\right)$ and $w C_{\xi}=C_{\xi^{\prime}}$.

(c) $w \in \Omega\left(M, M^{\prime}\right)$ and $w^{-1} \beta>0$ for all $\beta \in \Phi_{M^{\prime}}^{+} \cap\left(\mathfrak{A}_{M^{\prime}}^{*}\right)_{\xi^{\prime}}^{+}$.

Proof. The equivalence of (b) and (c) follows immediately from the definitions. To prove the equivalence of (a) and (c), assume first that $w=s_{\alpha}$ is an elementary symmetry. Then $w^{-1} \beta>0$ for all $\beta \in \Phi_{M^{\prime}}^{+} \cap\left(\mathfrak{A}_{M^{\prime}}^{*}\right)_{\xi^{\prime}}^{+}$if and only if $\alpha \notin\left(\mathfrak{A}_{M}^{*}\right)_{\xi}^{+}$. But $\alpha \notin\left(\mathfrak{A}_{M}^{*}\right)_{\xi}^{+}$if and only if $\xi \stackrel{\alpha}{\longrightarrow} \xi^{\prime}$. Notice that when (c) holds, $w^{-1}$ maps $\Phi_{M^{\prime}}^{+} \cap\left(\mathfrak{A}_{M^{\prime}}^{*}\right)_{\xi^{\prime}}^{+}$bijectively to $\Phi_{M}^{+} \cap\left(\mathfrak{A}_{M}^{*}\right)_{\xi}^{+}$.

Now assume (a) holds. Then we may write $w$ as a product $w=s_{\alpha_{k}} \cdots s_{\alpha_{1}}$ of elementary symmetries arising from a path in $\Gamma$. Set $w_{j}=s_{\alpha_{j-1}} \cdots s_{\alpha_{1}}, \xi_{j}=$ $w_{j} \xi w_{j}^{-1}$ and $M_{j}=w_{j} M w_{j}^{-1}$. By induction, $w_{k}^{-1}$ maps $\Phi_{M_{k}}^{+} \cap\left(\mathfrak{A}_{M_{k}}^{*}\right)_{\xi_{k}}^{+}$bijectively to $\Phi_{M}^{+} \cap\left(\mathfrak{A}_{M}^{*}\right)_{\xi}^{+}$and $s_{\alpha_{k}}^{-1}$ maps $\Phi_{M^{\prime}}^{+} \cap\left(\mathfrak{A}_{M^{\prime}}^{*}\right)_{\xi^{\prime}}^{+}$bijectively to $\Phi_{M_{k}}^{+} \cap\left(\mathfrak{A}_{M_{k}}^{*}\right)_{\xi_{k}}^{+}$. Hence $w^{-1}$ maps $\Phi_{M^{\prime}}^{+} \cap\left(\mathfrak{A}_{M^{\prime}}^{*}\right)_{\xi^{\prime}}^{+}$bijectively to $\Phi_{M}^{+} \cap\left(\mathfrak{A}_{M}^{*}\right)_{\xi}^{+}$and (c) holds.

Finally, assume (c) holds and let $w=s_{\alpha_{k}} \cdots s_{\alpha_{1}}$ be a reduced decomposition of $w$. Set $w_{j}=s_{\alpha_{j-1}} \cdots s_{\alpha_{1}}$. Since the decomposition is reduced, $w_{j}^{-1} \alpha_{j}>0$ and $w w_{j}^{-1} \alpha_{j}<0$ for $j=1, \ldots, k$ ([MW], p. 14). In particular, the $\operatorname{root} \beta=-s_{\alpha_{k}} \alpha_{k}$ lies in $\Phi_{M^{\prime}}^{+}$and $w^{-1} \beta=-w_{k}^{-1} \alpha_{k}<0$. Our hypothesis implies that $\beta \notin\left(\mathfrak{A}_{M^{\prime}}^{*}\right)_{\xi^{\prime}}^{+}$. Therefore $\xi_{k} \stackrel{\alpha_{k}}{\longrightarrow} \xi^{\prime}$ is an edge in $\Gamma$. It also follows that $w_{k}$ satisfies condition (c) with $M_{k}$ in place of $M^{\prime}$ and, by induction, $w_{k} \in \Omega\left(\xi, w_{k} \xi w_{k}^{-1}\right)$. Hence $w=s_{\alpha_{k}} w_{k}$ belongs to $\Omega\left(\xi, \xi^{\prime}\right)$ as required.

Corollary 35. Let $w \in \Omega\left(\xi, \xi^{\prime}\right)$. Then every reduced decomposition $w=s_{\alpha_{k}} \cdots s_{\alpha_{1}}$ is obtained from a word in the graph $\Gamma$.

Remark 4. Assume that $M=M_{0}$ and let $\left\{\beta_{1}, \ldots, \beta_{r}\right\}$ be the positive roots contained in $\left(\mathfrak{A}_{M}^{*}\right)_{\xi}^{-}$. It is straightforward to check that $\Omega(\xi, \xi) \cong(\mathbb{Z} / 2)^{r} \rtimes S_{r}$. The group $\Omega(\xi, \xi)$ acts by signed permutations on $\left(\mathfrak{A}_{0}^{*}\right)_{\xi}^{-}$, i.e., $S_{r}$ permutes the $\beta_{j}$ and the $j^{\text {th }}$ factor of $(\mathbb{Z} / 2)^{r}$ acts by sending $\beta_{j}$ to $-\beta_{j}$. The subgroup $\Omega_{\xi}^{+}$of $\Omega$ generated by reflections about roots contained in $\left(\mathfrak{A}_{0}^{*}\right)_{\xi}^{+}$is isomorphic to $S_{n-2 r}$ and

$$
\left\{w \in \Omega: w\left(\mathfrak{A}_{0}^{*}\right)_{\xi}^{-}=\left(\mathfrak{A}_{0}^{*}\right)_{\xi}^{-}\right\}=\Omega(\xi, \xi) \times \Omega_{\xi}^{+} .
$$

It is sufficient to check these facts for $\xi=(1,2) \cdots(2 r-1,2 r)$.

20. Unramified computations. In this section we consider a local analogue of the intertwining period in the unramified case. Assume that $n=2 m$ and let $P=M N$ be the standard parabolic subgroup of type $(m, m)$. We use the notation of Section $V I$. Thus $\xi$ is the non-trivial element in $\Omega(M, M)$ and $\eta \in G L_{n}(E)$ is such that $\eta \bar{\eta}^{-1}=\xi$. The subgroup $M_{\eta}$ consists of the matrices $\operatorname{diag}(h, \bar{h})$ where $h \in G L_{m}(E)$. 
Assume that $\sigma=\sigma_{1} \otimes \sigma_{2}$ is a cuspidal representation of $M_{E}\left(\mathbb{A}_{E}\right)$ trivial on $A_{M}$ such that $\sigma_{2} \simeq \bar{\sigma}_{1}^{*}$. There is a unique (up to scalars) non-zero linear form $L^{\prime}$ on the space of $\sigma$ which is invariant under $M_{\eta}(\mathbb{A})$, namely

$$
L^{\prime}(\varphi)=\int_{M_{\eta}(F) \backslash M_{\eta}(\mathbb{A})^{1}} \varphi(m) d m .
$$

For $j=1,2$, we choose an identification of $\sigma_{j}$ with a restricted tensor product $\otimes^{\prime} \sigma_{j v}$ where the product is over places $v$ of $F$. This identification presupposes the choice of $K_{v}$-fixed vectors $x_{j v}$ in the space of $\sigma_{j v}$ for almost all $v$. Thus, $\sigma_{j v}$ is a representation of $G L_{m}\left(E_{v}\right)$ where $E_{v}=E \otimes F_{v}$. We set $\sigma_{v}=\sigma_{1 v} \otimes \sigma_{2 v}$. Since $\sigma_{2 v} \simeq \bar{\sigma}_{1 v}^{*}$, there exists a non-zero linear map

$$
L_{v}^{\prime}: \sigma_{1 v} \otimes \sigma_{2 v} \longrightarrow \mathbb{C}
$$

invariant under $M_{\eta}$. It is also unique up to scalar multiples. We may assume that $L_{v}^{\prime}\left(x_{1 v} \otimes x_{2 v}\right)=1$ for almost all $v$. Then for a suitable normalization of $L^{\prime}$, we have

$$
L^{\prime}(\varphi)=\prod_{v} L_{v}^{\prime}\left(\varphi_{v}\right)
$$

whenever $\varphi$ corresponds to a pure tensor $\otimes \varphi_{v}$.

Set

$$
\pi_{v}=\operatorname{Ind}_{P_{v}}^{G_{v}} \sigma_{v}, \quad \pi=\operatorname{Ind}_{P(\mathbb{A})}^{G(\mathbb{A})} \sigma
$$

and let $V\left(\pi_{v}\right)$ and $V(\pi)$ denote the space of $\pi_{v}$ and $\pi$, respectively. If $\varphi_{v} \in V\left(\pi_{v}\right)$, then $\varphi_{v}(g)$ lies in the space of $\sigma_{1 v} \otimes \sigma_{2 v}$ for all $g \in G_{v}$ and hence we may define a function $L_{v}\left(\varphi_{v}\right)(g)$ whose value is the image of $\varphi_{v}(g)$ under $L_{v}^{\prime}$. This function is left-invariant under $M_{\eta}\left(E_{v}\right)$. Similarly, for $\varphi$ belonging to the space $V(\pi)$ of $\pi$, we may set $L(\varphi)(g)$ equal to the image of $\varphi(g)$ under $L^{\prime}$. Then

$$
L(\varphi)(g)=\prod_{v} L_{v}\left(\varphi_{v}\right)\left(g_{v}\right)
$$

In the global theory we used unnormalized induction. However, it will be more convenient to us to use normalized induction for the local computation. Thus, we now have

$$
J(\xi, \varphi, \lambda)=\int_{H_{\eta}(\mathbb{A}) \backslash H(\mathbb{A})} e^{\left\langle\lambda, H_{P_{E}}(\eta h)\right\rangle} L(\varphi)(\eta h) d h .
$$

(The $\rho_{P}$ is already absorbed in $\varphi$ because we use normalized induction.) Define the following local intertwining period:

$$
J_{v}\left(\xi, \varphi_{v}, \lambda\right)=\int_{H_{\eta}\left(F_{v}\right) \backslash H\left(F_{v}\right)} e^{\left\langle\lambda, H_{P_{E}}\left(\eta h_{v}\right)\right\rangle} L_{v}\left(\varphi_{v}\right)\left(\eta h_{v}\right) d h_{v}
$$

for $\lambda \in\left(\mathfrak{A}_{P}^{*}\right)_{\xi}^{-}$. Then we have the factorization (for a suitable normalization of measures):

$$
J(\xi, \varphi, \lambda)=\prod_{v} J_{v}\left(\xi, \varphi_{v}, \lambda\right)
$$

In this sense, the global intertwining period is equal to the product of the local intertwining periods. We shall now compute the local factor $J_{v}\left(\xi, \varphi_{v}, \lambda\right)$ in the unramified case.

For the rest of this section, assume that $v$ is a finite place such that $\sigma_{j v}$ is unramified for $j=1,2$. We shall show below that the integral converges absolutely 
for $\operatorname{Re} \lambda>>0$ and compute its value assuming that $\varphi_{v}$ is a $K_{v}$-fixed vector in $V\left(\pi_{v}\right)$. To this end, we recall the definition of the Asai $L$-function of an unramified representation of $G L_{m}(E)([\mathrm{AS}],[\mathrm{F} 1])$. We view $G L(m)_{E}$ as a group over $F$. Its $L$-group is then

$$
{ }^{L} G L(m)_{E}=G L_{m}(\mathbb{C}) \times G L_{m}(C) \rtimes G a l(E / F)
$$

where the non-trivial element $\sigma_{E / F} \in \operatorname{Gal}(E / F)$ acts on the connected component by interchanging the factors. Let $V=\mathbb{C}^{m}$ and let $T$ be the automorphism of $V \otimes V$ sending $x \otimes y$ to $y \otimes x$. We identify $G L(V \otimes V)$ with $G L_{m^{2}}(\mathbb{C})$ and define

$$
\rho_{A}:{ }^{L} G L(m, E) \longrightarrow G L_{m^{2}}(\mathbb{C}),
$$

where $\rho_{A}(g \times h \times 1)=g \otimes h$ and $\rho_{A}\left(1 \times 1 \times \sigma_{E / F}\right)=T$. Write $\omega_{E / F}$ for the character of ${ }^{L} G L(m)_{E}$ obtained by pulling back the non-trivial character of $\operatorname{Gal}(E / F)$. Then $\rho_{A} \otimes \omega_{E / F}$ is also defined. If $\sigma_{v}$ is an unramified representation, we may define the local Langlands $L$-factors $L\left(\lambda, \sigma_{v}, \rho_{A}\right)$ and $L\left(\lambda, \sigma_{v}, \rho_{A} \otimes \omega_{E / F}\right)$ attached to $\rho_{A}$ and $\rho_{A} \otimes \omega_{E / F}$ respectively. Note that the direct sum $\rho_{A} \oplus \rho_{A} \otimes \omega_{E / F}$ is isomorphic to the representation of ${ }^{L} G L(m)_{E}$ induced from the tensor product representation of $G L_{m}(\mathbb{C}) \times G L_{m}(C)$. Since formation of $L$-functions is invariant under induction, we obtain

$$
L\left(\sigma_{v}, s, \rho_{A}\right) L\left(\sigma_{v}, s, \rho_{A} \otimes \omega_{E / F}\right)=L\left(s, \sigma_{v} \times \overline{\sigma_{v}}\right)
$$

where $L\left(s, \sigma_{v} \times \overline{\sigma_{v}}\right)$ is the Rankin-Selberg convolution of $\sigma_{v}$ and $\overline{\sigma_{v}}$ ([JS]).

Actually, we shall need to use the contragredient representation $\rho_{A}^{*}$. Let us write down the local factors explicitly. Recall that $\sigma_{v}$ is a representation of $G L_{m}\left(E_{v}\right)$ where $E_{v}=E \otimes F_{v}$. If $v$ splits in $E$, then $G L_{m}\left(E_{v}\right)=G L_{m}\left(E_{w_{1}}\right) \times G L_{m}\left(E_{w_{2}}\right)$ and $\sigma_{v}=\sigma_{w_{1}} \otimes \sigma_{w_{2}}$ where $w_{1}$ and $w_{2}$ are the place of $E$ dividing $v$. For $j=1,2, \sigma_{w_{j}}$ corresponds to a Langlands class $g\left(\sigma_{w_{j}}\right) \in G L_{m}(\mathbb{C})$ and the Langlands class of $\sigma_{v}$ in ${ }^{L} G L(m, E)$ is $g\left(\sigma_{v}\right)=g\left(\sigma_{w_{1}}\right) \times g\left(\sigma_{w_{2}}\right) \times 1$. In this case,

$$
L\left(\lambda, \sigma_{v}, \rho_{A}^{*}\right)=L\left(\lambda, \sigma_{v}, \rho_{A}^{*} \otimes \omega_{E / F}\right)=\operatorname{det}\left(1-q_{v}^{-\lambda} g\left(\sigma_{w_{1}}\right)^{*} \otimes g\left(\sigma_{w_{2}}\right)^{*}\right)
$$

where $q_{v}$ is the order of the residue field of $F_{v}$ and $g^{*}={ }^{t} g^{-1}$. This is the local factor in the Rankin-Selberg product $\sigma_{w_{1}}^{*} \times \sigma_{w_{2}}^{*}$. If $v$ remains prime in $E$, let $w$ be the unique place of $E$ dividing $v$. Then $E_{v}$ is a field and $\sigma_{v}=\sigma_{w}$ corresponds to a Langlands class $g\left(\sigma_{w}\right) \in G L_{m}(\mathbb{C})$. It also corresponds to a Langlands class in ${ }^{L} G L(m, E)$, namely $g\left(\sigma_{w}\right) \times 1 \times \sigma_{E / F}$, and we have

$$
L\left(\lambda, \sigma_{v}, \rho_{A}^{*}\right)=\operatorname{det}\left(1-q_{v}^{-\lambda}\left(g\left(\sigma_{w}\right)^{*} \otimes 1\right) T\right) .
$$

If $g\left(\sigma_{w}\right)$ has eigenvalues $q_{w}^{\lambda_{1}}, \ldots, q_{w}^{\lambda_{n}}$ (where $q_{w}=q_{v}^{2}$ ), then

$$
\begin{aligned}
L\left(\lambda, \sigma_{v}, \rho_{A}^{*}\right) & =\prod_{1 \leq i<j \leq n}\left(1-q_{w}^{-\lambda_{i}-\lambda_{j}} q_{w}^{-\lambda}\right)^{-1} \prod_{i=1}^{n}\left(1-q_{w}^{-\lambda_{i}} q_{v}^{-\lambda}\right)^{-1}, \\
L\left(\lambda, \sigma_{v}, \rho_{A}^{*} \otimes \omega_{E / F}\right) & =\prod_{1 \leq i<j \leq n}\left(1-q_{w}^{-\lambda_{i}-\lambda_{j}} q_{w}^{-\lambda}\right)^{-1} \prod_{i=1}^{n}\left(1+q_{w}^{-\lambda_{i}} q_{v}^{-\lambda}\right)^{-1} .
\end{aligned}
$$

We now begin our computation of $J_{v}\left(\xi, \varphi_{v}, \lambda\right)$ for $\varphi_{v}$ fixed by $K_{v}$. Suppose that $\sigma_{1 v}$ is the unramified constituent of $\operatorname{Ind}_{B_{m}\left(E_{v}\right)}^{G L_{m}\left(E_{v}\right)} \chi$ where $B_{m}$ is the standard upper-triangular Borel subgroup of $G L(m)$ and $\chi=\left(\chi_{1}, \ldots, \chi_{m}\right)$ is an $m$ tuple of unramified characters of $E_{v}^{*}$. Then $\sigma_{2 v}$ is the unramified constituent 
of $\operatorname{Ind}_{B_{m}\left(E_{v}\right)}^{G L_{m}\left(E_{v}\right)} \chi^{-1}$. Let $\chi^{*}$ be the character of the upper-triangular Borel subgroup $B\left(E_{v}\right)$ of $G\left(E_{v}\right)$ defined by the $n$-tuple $\left(\chi_{1}, \ldots, \chi_{m}, \chi_{1}^{-1}, \ldots, \chi_{m}^{-1}\right)$. We identify $\operatorname{Ind}_{B_{m}\left(E_{v}\right)}^{G L_{m}\left(E_{v}\right)} \chi \otimes \operatorname{Ind}_{B_{m}\left(E_{v}\right)}^{G L_{m}\left(E_{v}\right)} \chi^{-1}$ with $\operatorname{Ind}_{B\left(E_{v}\right)}^{M\left(E_{v}\right)} \chi^{*}$. For $\psi$ in the space of $\operatorname{Ind}_{B\left(E_{v}\right)}^{M\left(E_{v}\right)} \chi^{*}$, set

$$
L_{v}^{\prime}(\psi)=\int_{B_{\eta}\left(F_{v}\right) \backslash M_{\eta}\left(F_{v}\right)} \psi(m) d m
$$

Here we use that the modulus function of $B_{\eta}\left(F_{v}\right)$ is equal to the restriction of the character $e^{\left\langle\rho_{B}, H_{B_{E}}(m)\right\rangle}$. This linear functional is $M_{\eta}\left(F_{v}\right)$-invariant and, up to multiples, it is the unique such functional. We may identify $\pi_{v}$ with the unramified constituent of the induced representation $\Sigma_{v}=\operatorname{Ind}_{B\left(E_{v}\right)}^{G\left(E_{v}\right)} \chi^{*}$ and on the induced space of $\Sigma_{v}$, the functional $L_{v}$ can be written

$$
L_{v}(\varphi)(g)=\int_{B_{\eta}\left(F_{v}\right) \backslash M_{\eta}\left(F_{v}\right)} \varphi(m g) d m
$$

where $d m$ is the semi-invariant measure on $B_{\eta} \backslash M_{\eta}\left(F_{v}\right)$. The local intertwining period can be written

$$
\begin{aligned}
J_{v}(\xi, \varphi, \lambda) & =\int_{H_{\eta}\left(F_{v}\right) \backslash H\left(F_{v}\right)} e^{\left\langle\lambda, H_{P_{E}}(\eta h)\right\rangle} L_{v}(\varphi)(\eta h) d h \\
& =\int_{H_{\eta}\left(F_{v}\right) \backslash H\left(F_{v}\right)} \int_{B_{\eta} \backslash M_{\eta}\left(F_{v}\right)} e^{\left\langle\lambda, H_{P_{E}}(\eta h)\right\rangle} \varphi(m \eta h) d m d h \\
& =\int_{B_{\eta}^{\prime}\left(F_{v}\right) \backslash H\left(F_{v}\right)} e^{\left\langle\lambda, H_{P_{E}}(\eta h)\right\rangle} \varphi(\eta h) d h
\end{aligned}
$$

where $B_{\eta}^{\prime}\left(F_{v}\right)=\eta^{-1} B_{\eta}\left(F_{v}\right) \eta=H\left(F_{v}\right) \cap \eta^{-1} B_{E}\left(E_{v}\right) \eta$.

The essential vector is the unique function $\varphi_{v}$ in the space $\Sigma_{v}$ which is rightinvariant under $G L_{n}\left(\mathcal{O}_{v}\right)$ (where $\mathcal{O}_{v}$ is the ring of integers in $E_{v}$ ) and satisfies $\varphi_{v}(e)=1$. We identify $\mathfrak{A}_{P, \mathbb{C}}^{*}$ with $\mathbb{C}$ by sending $\varpi$ to 1 , where $\hat{\Delta}_{P}=\{\varpi\}$.

Theorem 36. Assume that $v \notin S$ and let $\varphi_{v}$ be the essential vector. For a suitable normalization of measures we have

$$
J_{v}\left(\xi, \varphi_{v}, \lambda\right)=\frac{L\left(\lambda, \sigma_{1 v}, \rho_{A}^{*}\right)}{L\left(\lambda+1, \sigma_{1 v}, \rho_{A}^{*} \otimes \omega_{E / F}\right)} .
$$

We first prove this theorem in the case that $v$ splits in $E$. Then we may identify $G\left(E_{v}\right)$ with $G L_{n}\left(E_{w_{1}}\right) \times G L_{n}\left(E_{w_{2}}\right)$ where $w_{1}, w_{2}$ are the places of $E$ dividing $v$. We have $\sigma_{j v}=\sigma_{j w_{1}} \otimes \sigma_{j w_{2}}$ where $\sigma_{1 w_{1}} \simeq \sigma_{2 w_{2}}^{*}$ and $\sigma_{1 w_{2}} \simeq \sigma_{2 w_{1}}^{*}$. Conjugation acts by $(x, y) \longrightarrow(y, x)$ and $H\left(F_{v}\right)$ is imbedded diagonally. We may also take $\eta=(1, \xi)$. Then $H_{\eta}\left(F_{v}\right)$ is the Levi factor of the parabolic subgroup $P=M N$ type $(m, m)$ in $H\left(F_{v}\right)$ and $B_{\eta}^{\prime}\left(F_{v}\right)=B\left(F_{v}\right) \cap H_{\eta}\left(F_{v}\right)$. Using the Iwasawa decomposition 


$$
\begin{aligned}
H\left(F_{v}\right)=B_{\eta}^{\prime}\left(F_{v}\right) N\left(F_{v}\right) & \mathbf{K}_{F} \text { we obtain } \\
J_{v}(\xi, \varphi, \lambda) & =\int_{B_{\eta}^{\prime}\left(F_{v}\right) \backslash H\left(F_{v}\right)} e^{\left\langle\lambda, H_{P}(\eta h)\right\rangle} \varphi(\eta h) d h \\
& =\int_{B_{\eta}^{\prime}\left(F_{v}\right) \backslash H\left(F_{v}\right)} e^{\left\langle\lambda, H_{P}(h)+H_{P}(\xi h)\right\rangle} \varphi_{1}(h) \varphi_{2}(\xi h) d h \\
& =\int_{N\left(F_{v}\right)} e^{\left\langle\lambda, H_{P}(\xi n)\right\rangle} \varphi_{2}(\xi n) d n .
\end{aligned}
$$

In other words, $J_{v}\left(\xi, \varphi_{v}, \lambda\right)$ coincides with the standard intertwining operator applied to the essential vector in $\operatorname{Ind}_{P_{w_{2}}}^{G_{w_{2}}}\left(\sigma_{1 w_{2}} \otimes \sigma_{2 w_{2}}\right)$. By the Gindikin-Karpelevic formula, the integral is equal to

$$
\frac{L\left(\lambda, \sigma_{1 w_{2}}^{*} \otimes \sigma_{2 w_{2}}\right)}{L\left(\lambda+1, \sigma_{1 w_{2}}^{*} \otimes \sigma_{2 w_{2}}\right)}=\frac{L\left(\lambda, \sigma_{1 w_{2}}^{*} \otimes \sigma_{1 w_{1}}^{*}\right)}{L\left(\lambda+1, \sigma_{1 w_{2}}^{*} \otimes \sigma_{1 w_{1}}^{*}\right)}
$$

and this is equal to $L\left(\lambda, \sigma_{1 v}^{*}, \rho_{A}\right) / L\left(\lambda+1, \sigma_{1 v}^{*}, \rho_{A} \otimes \omega_{E / F}\right)$ as claimed.

We now suppose that $v$ remains prime in $E$, and, for simplicity, we drop $v$ from the notation. We first consider the case $H=G L_{2}(F)$ and $G=G L_{2}(E)$ where $E / F$ is an unramified extension of $p$-adic fields. We also assume that $p \neq 2$. We write $|\cdot|$ and $\|\cdot\|$ for the absolute values on $F$ and $E$, respectively, $q$ for the order of the residue field of $F$, and $q_{E}=q^{2}$ for the order of the residue field of $E$. Fix $i \in E^{*}$ such that $\operatorname{Tr}_{E / F}(i)=0$ and set

$$
\eta=\left(\begin{array}{cc}
1 & i \\
1 & -i
\end{array}\right)
$$

Then

$$
\eta \bar{\eta}^{-1}=\left(\begin{array}{cc}
1 & i \\
1 & -i
\end{array}\right)\left(\begin{array}{cc}
\frac{1}{2} & \frac{1}{2} \\
\frac{-1}{2 i} & \frac{1}{2 i}
\end{array}\right)=\left(\begin{array}{cc}
0 & 1 \\
1 & 0
\end{array}\right)
$$

and the subgroup $T=H \cap \eta^{-1} B(E) \eta$ is a torus isomorphic to $E^{*}$. We shall compute

$$
\int_{T \backslash H} \varphi(\eta h) d h
$$

where

$$
\varphi\left(\left(\begin{array}{cc}
a & * \\
0 & b
\end{array}\right) k\right)=\left\|\frac{a}{b}\right\|^{\frac{\lambda+1}{2}} .
$$

Thus $\varphi$ is the essential vector in the unramified representation $G L_{2}(E)$ with Langlands class

$$
\left(\begin{array}{cc}
q_{E}^{\frac{\lambda}{2}} & 0 \\
0 & q_{E}^{-\frac{\lambda}{2}}
\end{array}\right) \in G L_{2}(\mathbb{C}) .
$$

To fix the quotient measure on $T \backslash H$, we observe that every $h \in H$ can be written uniquely in the form

$$
h=t\left(\begin{array}{ll}
1 & b \\
0 & 1
\end{array}\right)\left(\begin{array}{cc}
a & 0 \\
0 & 1
\end{array}\right)
$$

with $t \in T$. In these coordinates, a right-invariant measure on $T \backslash H$ is given by $\frac{d a}{|a|} d b$. Assume that the measures $d a$ and $d b$ assign measure 1 to the ring of integers 
$\mathcal{O}_{F}$. With this normalization, we have the following result. It is proved in [JL] using the method of Lemma 27 above. Here we give a direct computational proof.

Proposition 37. We have

$$
\int_{T \backslash H} \varphi(\eta h) d h=\|i\|^{\frac{1}{2}} \frac{1+q^{-\lambda-1}}{1-q^{-\lambda}} .
$$

Proof. Since

$$
\eta\left(\begin{array}{ll}
1 & b \\
0 & 1
\end{array}\right)\left(\begin{array}{cc}
a & 0 \\
0 & 1
\end{array}\right)=\left(\begin{array}{ll}
a & b+i \\
a & b-i
\end{array}\right)
$$

we have

$$
\begin{aligned}
\varphi\left(\left(\begin{array}{cc}
a & b+i \\
a & b-i
\end{array}\right)\right) & =\|2 a i\|^{\frac{\lambda+1}{2}}(\max \{\|a\|,\|b-i\|\})^{-\lambda-1} \\
& =\|i\|^{\frac{\lambda+1}{2}}\|a\|^{\frac{\lambda+1}{2}} \max \{\|a\|,\|i-b\|\}^{-\lambda-1}
\end{aligned}
$$

and hence

$$
\int_{T \backslash H} \varphi(\eta h) d h=\|i\|^{\frac{\lambda+1}{2}} \int_{F^{*}} f(a)\|a\|^{\frac{\lambda+1}{2}} \frac{d a}{|a|}
$$

where

$$
f(a)=\int_{F} \max \{\|a\|,\|i-b\|\}^{-\lambda-1} d b .
$$

We now compute $f(a)$. Set

$$
X=1+\int_{|b|>1}|b|^{-2 \lambda-1} \frac{d b}{|b|} .
$$

It is easily checked that

$$
X=\frac{1-q^{-2 \lambda-2}}{1-q^{-2 \lambda-1}}
$$

with the normalization $\operatorname{vol}\left(\mathcal{O}_{F}\right)=1$.

Lemma 38. $f(a)=|a|^{-2 \lambda-1} X$ if $\|a\| \geq\|i\|$ and $f(a)=\|i\|^{-\lambda-\frac{1}{2}} X$ if $\|a\|<\|i\|$.

Proof. If $\|a\| \geq\|i\|$, then

$$
\begin{aligned}
f(a) & =\int_{|b| \leq|a|}\|a\|^{-\lambda-1} d b+\int_{|b|>|a|} \|\left. b\right|^{-\lambda-1} d b \\
& =|a|^{-2 \lambda-2} \operatorname{vol}(\{b:|b| \leq|a|\})+|a|^{-2 \lambda-1} \int_{|b|>1}|b|^{-2 \lambda-1} \frac{d b}{|b|} \\
& =|a|^{-2 \lambda-1} X .
\end{aligned}
$$

If $\|a\|<\|i\|$, then

$$
\begin{aligned}
f(a) & =\int_{F}\|i-b\|^{-\lambda-1} d b \\
& =\|i\|^{-\lambda-1} \operatorname{vol}(\{b:\|b\| \leq\|i\|\})+\int_{\|b\|>\|i\| \mid}|b|^{-2 \lambda-1} \frac{d b}{|b|} \\
& =\|i\|^{-\lambda-\frac{1}{2}}+\|i\|^{-\lambda-\frac{1}{2}} \int_{|b|>1}|b|^{-2 \lambda-1} \frac{d b}{|b|} \\
& =\|i\|^{-\lambda-\frac{1}{2}} X .
\end{aligned}
$$


Now we have

$$
\begin{array}{rl}
\int_{F^{*}} & f(a)\|a\|^{\frac{\lambda+1}{2}} \frac{d a}{|a|}=\sum_{n=-\infty}^{\infty} f\left(p^{n}\right) q^{-n(\lambda+1)} \\
= & q^{r(2 \lambda+1)} X \sum_{n>r} q^{-n(\lambda+1)}+X \sum_{n \leq r} q^{n(2 \lambda+1)} q^{-n(\lambda+1)}
\end{array}
$$

and it is easily checked that this equals

$$
q^{r \lambda}\left(\frac{1+q^{-\lambda-1}}{1-q^{-\lambda}}\right)
$$

and the result follows.

The next proposition is the inert case of Theorem 36 .

Proposition 39. Let $E / F$ be an unramified quadratic extension of p-adic fields and let $\sigma_{1}$ be an unramified representation of $G L_{m}(E)$. For the unique normalized $K$-invariant $\varphi \in I_{P}\left(\sigma_{1} \times \bar{\sigma}_{1}^{*}\right)$ we have

$$
J(\xi, \varphi, \lambda)=\frac{L\left(\lambda, \sigma_{1}, \rho_{A}^{*}\right)}{L\left(\lambda+1, \sigma_{1}, \rho_{A}^{*} \otimes \omega_{E / F}\right)} .
$$

Proof. In the above notation, suppose that $\sigma_{1}=\operatorname{Ind}_{B_{m}(E)}^{G L_{m}(E)} \chi$ where $\chi=\left(\chi_{1}, \ldots, \chi_{m}\right)$ with $\chi_{i}=|\cdot|_{E}^{\lambda_{i}}$. As before, $\sigma_{2 v}=\operatorname{Ind}_{B_{m}\left(E_{v}\right)}^{G L_{m}\left(E_{v}\right)} \chi^{-1}$ and we view $\varphi$ as an element of $\operatorname{Ind}_{B_{n v}}^{G_{n v}}\left(\chi_{1}, \ldots, \chi_{n}, \chi_{1}^{-1}, \ldots, \chi_{n}^{-1}\right)$. To compute

$$
J_{v}(\xi, \varphi, \lambda)=\int_{B_{\eta}^{\prime}\left(F_{v}\right) \backslash H\left(F_{v}\right)} e^{\left\langle\lambda, H_{B_{E}}(\eta h)\right\rangle} \varphi(\eta h) d h,
$$

we shall regard it as a local intertwining period for an Eisenstein series induced from the Borel subgroup and reduce to the case $n=2$ by making use of the functional equations. Let $\xi^{\prime}=(1,2) \cdots(2 n-1,2 n)$. Then $\xi^{\prime}$ is a minimal involution and $\xi=w^{-1} \xi^{\prime} w$ where $w$ is defined by $w(i)=2 i-1$ and $w(i+n)=2 i$ for $i=1, \ldots, n$. Then $w$ has the following reduced decomposition:

$$
w=\left(s_{2 n-2}\right)\left(s_{2 n-4} s_{2 n-3}\right) \cdots\left(s_{4} \cdots s_{n} s_{n+1}\right)\left(s_{2} \cdots s_{n-1} s_{n}\right) .
$$

We observe that an analogue of Proposition 33 holds in the local case. It is proved in the same way, by re-writing the absolutely convergent integral. Therefore

$$
J_{v}(\xi, \varphi, \lambda)=J_{v}\left(\xi^{\prime}, M(w, \lambda) \varphi, w \lambda\right) .
$$

As in the proof of Lemma 32, the right hand side can be written as a local intertwining period with respect to the group $G L_{2} \times \cdots \times G L_{2}$ ( $m$ times) and the induction from $\left(\chi_{1}, \chi_{1}^{-1}, \ldots, \chi_{m}, \chi_{m}^{-1}\right)$. By Proposition 37 we have

$$
J\left(\xi^{\prime}, \varphi, \lambda\right)=\prod_{i=1}^{n}\left(1-q_{E}^{-\lambda_{i}} q_{F}^{-\lambda}\right)^{-1}\left(1+q_{E}^{-\lambda_{i}} q_{F}^{-(\lambda+1)}\right) .
$$

By the formula of Gindikin and Karpelevic, $M(w, \lambda) \varphi=c(\lambda) \varphi$ where

$$
c(\lambda)=\prod_{1 \leq i<j \leq n} \frac{\left(1-q_{E}^{-\lambda_{i}-\lambda_{j}} q_{E}^{-\lambda}\right)^{-1}}{\left(1-q_{E}^{-\lambda_{i}-\lambda_{j}} q_{E}^{-(\lambda+1)}\right)^{-1}} .
$$

This completes the proof. 


\section{Periods of trunchted Eisenstein Series}

The next theorem gives our formula for $\Pi^{G / H}\left(\Lambda_{m}^{T} E(\varphi, \lambda)\right)$. Let $P$ be a parabolic subgroup, let $\left(n_{1}, \ldots, n_{r}\right)$ be the corresponding partition of $n$, and fix a cuspidal representation $\sigma$ of $M_{P_{E}}\left(\mathbb{A}_{E}\right)^{1}$. Let $\mathcal{G}(P, \sigma)$ be the set of pairs $(Q, s)$ consisting of a parabolic subgroup $Q$ and an element $s \in \Omega\left(M_{P}, M_{P^{\prime}}\right)$ with $P^{\prime} \leq Q$ such that $s^{-1} \alpha>0$ for all $\alpha \in \Delta_{P^{\prime}}^{Q}$ and there exists a set $X \subset\{1,2, \ldots, r-1\}$ of indices satisfying conditions (1), (2), (3) below. Let $\left(n_{1}^{\prime}, \ldots, n_{r}^{\prime}\right)$ be the partition corresponding to $M^{\prime}=M_{P^{\prime}}$, and set $s \sigma=\sigma^{\prime}=\sigma_{1}^{\prime} \otimes \sigma_{2}^{\prime} \otimes \cdots \otimes \sigma_{r}^{\prime}$. Then

(1) For all $j \in X$ we have $j+1 \notin X, n_{j}^{\prime}=n_{j+1}^{\prime}$ and $\left(\sigma_{j+1}^{\prime}\right)^{*} \simeq \overline{\sigma_{j}^{\prime}}$.

(2) For all $j$ such that $j, j-1 \notin X, \sigma_{j}^{\prime}$ is distinguished with respect to $G L_{n_{j}^{\prime}}\left(\mathbb{A}_{F}\right)$.

(3) $Q$ corresponds to the partition obtained from $\left(n_{1}^{\prime}, \ldots, n_{r}^{\prime}\right)$ by replacing the pair of entries $n_{j}^{\prime}, n_{j+1}^{\prime}$ by the single entry $n_{j}^{\prime}+n_{j+1}^{\prime}$ for all $j \in X$.

Let $\mathcal{G}_{P}(\sigma)$ be the set of all parabolic subgroups $Q$ that appear in some pair $(Q, s) \in$ $\mathcal{G}(P, \sigma)$.

The Levi subgroup $M^{\prime}$ of $P^{\prime}$ is isomorphic to $G L_{n_{1}^{\prime}} \times \cdots \times G L_{n_{r}^{\prime}}$. Let $\xi_{Q}$ be the unique element in $\Omega\left(M^{\prime}, M^{\prime}\right)$ such that $\xi$ interchanges the $j^{\text {th }}$ and $(j+1)^{t h}$ factors of $M^{\prime}$ for $j \in X$ and fixes all other factors. We write $\lambda=\lambda_{Q}+\lambda_{P^{\prime}}^{Q}$ for the decomposition of an element $\lambda \in \mathfrak{A}_{P^{\prime}}^{*}$ relative to the direct sum $\mathfrak{A}_{P^{\prime}}^{*}=\mathfrak{A}_{Q}^{*} \oplus\left(\mathfrak{A}_{P^{\prime}}^{Q}\right)^{*}$. Let

$$
v_{Q}=2^{d(H)-d(Q)} \operatorname{vol}\left\{\sum_{\alpha \in \Delta_{Q}} a_{\alpha} \alpha^{\vee}: 0 \leq a_{\alpha} \leq 1\right\} .
$$

Theorem 40. Let $\varphi \in \mathcal{A}_{P_{E}}(G)_{\sigma}$ where $\sigma$ is a cuspidal representation of $M_{E}\left(\mathbb{A}_{E}\right)$, and let $E(g, \varphi, \lambda)$ be the associated Eisenstein series. Then as a meromorphic function of $\lambda$,

$$
\int_{H(F) \backslash H(\mathbb{A})^{1}} \Lambda_{m}^{T} E(h, \varphi, \lambda) d h
$$

is equal to

$$
\sum_{(Q, s) \in \mathcal{G}(P, \sigma)} v_{Q} \frac{e^{\left\langle(s \lambda)_{Q}, T\right\rangle}}{\prod_{\alpha \in \Delta_{Q}}\left\langle(s \lambda)_{Q}, \alpha^{\vee}\right\rangle} J\left(\xi_{Q}, M(s, \lambda) \varphi,(s \lambda)_{P^{\prime}}^{Q}\right) .
$$

Proof. Since $\sigma$ is cuspidal, $E_{Q_{E}}(g, \varphi, \lambda)$ vanishes unless $Q$ contains an associate of $P$ and we obtain

$$
\begin{gathered}
\int_{H(F) \backslash H(\mathbb{A})^{1}} \Lambda_{m}^{T} E(g, \varphi, \lambda) d g \\
=\sum_{Q}(-1)^{d(Q)-d(H)} \int_{Q(F) \backslash H(\mathbb{A})^{1}}^{*} E_{Q_{E}}(g, \varphi, \lambda) \hat{\tau}_{Q}\left(H_{Q_{E}}(g)-T\right) d g
\end{gathered}
$$

where $Q$ ranges over such parabolics. Let $\Omega(P, Q)$ be the union over all $P^{\prime} \subset Q$ of the subset of elements $s \in \Omega\left(M_{P}, M_{P^{\prime}}\right)$ such that $s^{-1} \alpha>0$ for all $\alpha \in \Delta_{P^{\prime}}^{Q}$. Then

$$
E_{Q_{E}}(g, \varphi, \lambda)=\sum_{s \in \Omega(P, Q)} E^{Q_{E}}(g, M(s, \lambda) \varphi, s \lambda)
$$


where $E^{Q_{E}}(g, M(s, \lambda) \varphi, \lambda)$ is the Eisenstein series on $Q_{E}$ induced from the function $M(s, \lambda) \varphi$. We must therefore compute the integrals

$$
\int_{Q(F) \backslash H(\mathbb{A})^{1}}^{*} E^{Q_{E}}(g, M(s, \lambda) \varphi, s \lambda) \hat{\tau}_{Q}\left(H_{Q_{E}}(g)-T\right) d g
$$

for $s \in \Omega(P, Q)$. By definition, (67) is equal to

$$
\Pi^{M_{Q_{E}} / M_{Q}}\left(E^{Q_{E}}\left(\cdot,(M(s, \lambda) \phi)^{\mathbf{K}_{F}},(s \lambda)_{P^{\prime}}^{Q}\right)\right) \int_{\mathfrak{A}_{Q}}^{*} e^{\left\langle(s \lambda)_{Q}, 2 X\right\rangle} \hat{\tau}_{Q}(2 X-T) d X
$$

where we have set:

$$
(M(s, \lambda) \phi)^{\mathbf{K}_{F}}(m)=\int_{\mathbf{K}_{F}} M(s, \lambda) \phi(m k) d k
$$

and $E^{Q_{E}}$ is an Eisenstein series for the parabolic subgroup $P_{E} \cap M_{Q_{E}}$ of the group $M_{Q_{E}}$. By Theorem 23 (applied to a product of linear groups), the first factor in (67) vanishes unless $(Q, s)$ belongs to $\mathcal{G}(P, \sigma)$. If $(Q, s) \in \mathcal{G}(P, \sigma)$, then it is equal to the following intertwining period integral for the group $M_{Q}$ :

$$
J^{M_{Q}}\left(\xi_{Q},(M(s, \lambda) \phi)^{\mathbf{K}_{F}},(s \lambda)_{P^{\prime}}^{Q}\right) .
$$

As in the proof of Lemma 32, this is the same as the following intertwining period for the group $G$ :

$$
J\left(\xi_{Q}, M(s, \lambda) \phi,(s \lambda)_{P^{\prime}}^{Q}\right)
$$

On the other hand,

$$
\int_{\mathfrak{A}_{Q}}^{*} e^{\left\langle(s \lambda)_{Q}, 2 X\right\rangle} \hat{\tau}_{Q}(2 X-T) d X=v_{Q} \frac{e^{\left\langle(s \lambda)_{Q}, T\right\rangle}}{\prod_{\alpha \in \Delta_{Q}}\left\langle(s \lambda)_{Q}, \alpha^{\vee}\right\rangle} .
$$

The required formula follows.

As an example, consider the case $P=B$, the Borel subgroup. Then the integral (67) vanishes unless $Q$ is a parabolic of type $\left(m_{1}, \ldots, m_{k}\right)$ with $m_{j}=1$ or 2 . Thus $M_{Q}$ is isomorphic to a product of copies of $G L_{1}$ and $G L_{2}$. Given such a $Q$, let $\xi_{Q}$ be a representative for the longest element in the Weyl group of $M_{Q}$, and let $\eta=\eta_{Q} \in Q(E)$ be such that $\eta \bar{\eta}^{-1}=\xi_{Q}$. Then we have

Proposition 41. Let $\sigma$ be a character of $T_{E}(E) \backslash T_{E}\left(\mathbb{A}_{E}\right)^{1}$ and let $E(g, \varphi, \lambda)$ be an Eisenstein series where $\varphi \in \mathcal{A}_{B_{E}}(G)_{\sigma}$. Then

$$
\int_{H(F) \backslash H(\mathbb{A})^{1}} \Lambda_{m}^{T} E(h, \varphi, \lambda) d h
$$

is equal to the sum of the terms

$$
v_{Q} \frac{e^{\left\langle(w \lambda)_{Q}, T\right\rangle}}{\prod_{\alpha \in \Delta_{Q}}\left\langle(w \lambda)_{Q}, \alpha^{\vee}\right\rangle} J\left(\xi_{Q}, M(w, \lambda) \varphi,(w \lambda)_{0}^{Q}\right)
$$


where $Q$ ranges over the parabolic subgroups of type $\left(n_{1}, \ldots, n_{r}\right)$ with $n_{j}=1$ or 2 and $w$ ranges over the elements of the Weyl group $\Omega$ such that $w \sigma$ is trivial on $T_{\eta_{Q}}(\mathbb{A})^{1}$, and $w^{-1} \alpha>0$ for $\alpha \in \Delta_{0}^{Q}$.

In this proposition, the intertwining integral $J\left(\xi_{Q}, M(w, \lambda) \varphi, w \lambda_{0}^{Q}\right)$ reduces to

$$
\operatorname{vol}\left(H_{\eta_{Q}}(F) A_{M_{Q}} \backslash H_{\eta_{Q}}(\mathbb{A})\right) \int_{H_{\eta_{Q}}(\mathbb{A}) \backslash H(\mathbb{A})} e^{\left\langle(w \lambda)_{0}^{Q}+\rho_{B}, H\left(\eta_{Q} h\right)\right\rangle} M(w, \lambda) \varphi\left(\eta_{Q} h\right) d h .
$$

Example 6. Consider the case $G=G L(2)$, and $E(g, \varphi, \lambda)$ is an Eisenstein series induced from a character $\sigma$ of $B_{E}(F) \backslash B_{E}\left(\mathbb{A}_{E}\right)$ trivial on $A_{0}$. Here we identify $\mathfrak{A}_{0, \mathbb{C}}^{*}$ with $\mathbb{C}$ by sending the fundamental weight to 1 and thus we view $\lambda$ as a complex parameter. Let $w$ be the non-trivial element in the Weyl group. There are three possible pairs $(Q, w):(B, e),(B, w)$, and $(G, e)$. If $Q=B$, then $\xi=\eta=e$ and $B_{\eta}=H_{\eta}=B$. The pairs $(B, e)$ and $(B, w)$ occur if and only if $\sigma$ is trivial on $B\left(\mathbb{A}_{F}\right)$. Identifying $\sigma$ with a pair of Hecke characters $\left(\sigma_{1}, \sigma_{2}\right)$ of $\mathbb{A}_{E}^{*}$, the condition is that $\sigma_{1}$ and $\sigma_{2}$ are trivial on $\mathbb{A}_{F}^{*}$. If so, the contribution of the pairs $(B, e)$ and $(B, w)$ is equal to $\frac{1}{2} \operatorname{vol}\left(T(F) A_{0} \backslash T(\mathbb{A})\right)$ times

$$
\frac{e^{\lambda T}}{\lambda} \int_{\mathbf{K}_{F}} \varphi(k) d k+\frac{e^{-\lambda T}}{-\lambda} \int_{\mathbf{K}_{F}} M(w, \lambda) \varphi(k) d k .
$$

If $Q=G$, then $\xi=w$ and $B_{\eta}$ is the torus

$$
B_{\eta}=\left\{\left(\begin{array}{cc}
\alpha & 0 \\
0 & \bar{\alpha}
\end{array}\right): \alpha \in E^{*}\right\}
$$

The pair $(G, e)$ contributes if and only if $\sigma_{2}(\alpha)=\sigma_{1}(\bar{\alpha})^{-1}$. If so, the contribution is

$$
\operatorname{vol}\left(B_{\eta}(F) A_{0} \backslash B_{\eta}(\mathbb{A})\right) \int_{H_{\eta}(\mathbb{A}) \backslash H(\mathbb{A})} e^{\langle\lambda+1, H(\eta h)\rangle} \varphi(\eta h) d h .
$$

\section{REFERENCES}

[A1] J. Arthur, A trace formula for reductive groups I, Duke Math. J., 45, No. 1 (1978), 911-952. MR 80d:10043

[A2] J. Arthur, A trace formula for reductive groups II, Compositio Math., 40 (1980), 87-121. MR 81b:22018

[A3] J. Arthur, The trace formula in invariant form, Duke Math. J., 114 (1981), 1-74. MR 84a:10031

[A4] J. Arthur, On the inner product of truncated Eisenstein series, Duke Math. J., 49 (1982), 35-70. MR 83e:22023

[AS] T. Asai, On certain Dirichlet series associated with Hilbert modular forms and Rankin's method, Math. Ann. 266 (1977), 81-94. MR 55:2761

[BZ] I. Bernstein and A. Zelevinsky, Induced representations of reductive $\mathfrak{p}$-adic groups I, Ann. scient. Éc. Norm. Sup., $4^{e}$ série, t. 10, 1977, p. 441-472. MR 58:28310

[BR] D. Blasius and J. Rogawski, Cohomology of congruence subgroups of $S U(2,1)^{p}$ and Hodge cycles on some special complex hyperbolic surfaces, to appear in: Proc. of conference on Regulators, Jerusalem 1996 (Ed. A. Resnikov and N. Schappacher).

[C] W. Casselman, Extended automorphic forms on the upper half-plane, Math. Annalen 296 (1993), 755-762. MR 94k:11049

[F1] Y. Flicker, Twisted Tensor and Euler Products, Bull. Soc. Math. France, 52 (1989), 295-313. MR 89m:11049 
[F2] Y. Flicker, On distinguished representations, J. Reine Angew. Math. 418 (1991), 139-172. MR 92i:22019

[F3] Y. Flicker, Distinguished Representations and a Fourier Trace Formula, Bull. Soc. Math. France, 120 (1992), 413-465. MR 93j:22033

[F4] Y. Flicker, Cyclic automorphic forms on a unitary group, J. Math. Kyoto Univ. 37 (1997), 367-439. CMP 98:07

[FH] Y. Flicker and J. Hakim, Quaternionic Distinguished Representations, American J. Math., 116 (1994), 255-278. MR 95i:22028

[FJ] S. Friedberg and H. Jacquet, Linear periods, J. Reine Angew. Math. 443 (1993), 91-139. MR 94k:11058

[FGJR] S. Friedberg, S. Gelbart, H. Jacquet, and J. Rogawski, On genericity for U(3) L-packets, pre-print.

[GJR] S. Gelbart, H. Jacquet, and J. Rogawski, The relative trace formula and genericity of $L$-packets for $U(3)$. In preparation.

[GRS] S. Gelbart, D. Soudry, and J. Rogawski, Endoscopy, theta-liftings, and period integrals for the unitary group in three variables. Annals of Math. 145 (1997), 1-58. CMP 97:14

[GU] J. Guo, On the positivity of central critical values of automorphic L-functions of GL(2), Duke Math. J., 83 (1996), 157-190. MR 97h:11055

[G] N. Gurevitch, Unpublished notes.

[HLR] G. Harder, R. Langlands, and M. Rapoport, Algebraische zyklen auf Hilbert-Blumenthal Flächen, J. reine angew. Math. 366 (1986), 53-120. MR 87k:11066

[J1] H. Jacquet, Sur un résultat de Waldspurger, Ann. Scient. Ec. Norm. Sup., 19 (1986), 185-229. MR 88d:11051

[J2] H. Jacquet, The continuous spectrum of the relative trace formula for GL(3) over a quadratic extension, Israel J. Math., 89 (1995), 1-59. MR 96a:22029

[J3] H. Jacquet, Automorphic spectrum of symmetric spaces, In: Representation Theory and Automorphic Forms, (Eds. T. N. Bailey and A. W. Knapp) Proc. Symposia in Pure Math. 61, AMS, Providence, 1997. CMP 98:03

[JLR] H. Jacquet, K. Lai, S. Rallis, A trace formula for symmetric spaces, Duke Math. J., 70, No. 2 (1993), 305-372. MR 94d:11033

[JL] H. Jacquet and K. Lai, A relative trace formula, Comp. Math., 54 (1985), 243-310. MR $\mathbf{8 6 \mathbf { j }}: 11059$

[JR] H. Jacquet and S. Rallis, Symplectic periods, J. Reine Angew. Math., 423 (1992), 175197. MR 93b:22035

[JS] H. Jacquet and J. Shalika, On Euler products and the classification of automorphic representations I,II, Amer. J. Math. 103 (1981), 499-558 and 777-815. MR 82m:10050a; MR 82m:10050b

[JY] H. Jacquet and Y. Ye, Une remarque sur le changement de base quadratique, C.R. Acad. Sci. Paris Sér. I , Math. 311 (1990), 671-676. MR 92j:11046

[JI] D. Jiang, Periods of residual representations of $G_{2}$, , pre-print.

[LA1] K. F. Lai, Algebraic Cycles on Compact Shimura Surface, Math. Z. 189 (1985), 593-602. MR 87a: 11057

[LA2] K. F. Lai, On Arthur's Class Expansion of the Relative Trace Formula, Duke Math. J. 64 (1991), 111-117. MR 92k:22031

[L] R. Langlands, Eisenstein series, in Algebraic Groups and Discontinuous Subgroups, Proc. Symp. Pure Math. vol. 9, AMS 1966. MR 40:2784

[LP] E. Lapid, Thesis, Weizmann Institute of Science, 1998.

[LE] J. Levy, A note on the relative trace formula, Canad. Math. Bull. vol. 38 (1995), 450-461. MR 97d: 11085

[LR] G. Laumon and M. Rapoport, The Langlands lemma and the Betti numbers of stacks of G-bundles on a curve, Internat. J. Math. 7 (1996), 29-45. MR 97f:14012

[MA] Z. Mao, Relative Kloosterman integrals for the unitary group, C.R. Acad. Sci. Paris Sér. I Math 315 (1992), 381-386. MR 93g:11051

[MW] C. Moeglin and J.-L. Waldspurger, Decomposition spectrale et series D'Eisenstein, Birkhauser 1993. MR 95d:11067

[SA] P. Sarnak, Diophantine problems and linear groups, In: Proceedings of Int. Cong. Math., Kyoto 1990, Springer Verlag 1991, 459-471. MR 93g:11054 
[S] T. Springer, Some results on algebraic groups with involutions, in Algebraic groups and related topics, Advanced Studies in Pure Mathematics, vol. 6, 1985, 525-543. MR 86m:20050

[Z] D. Zagier, The Rankin-Selberg method for automorphic functions which are not of rapid decay, J. Fac. Sci. Univ. Tokyo 28 (1982), 415-437. MR 83k:10056

Department of Mathematics, Columbia University, New York, New York 10027

E-mail address: hj@math.columbia.edu

Department of Mathematics, Weizmann Institute, Rehovot, Israel

E-mail address: erezl@wisdom.weizmann.ac.il

Department of Mathematics, Hebrew University, Jerusalem, Israel

E-mail address: jonr@math.huji.ac.il

Current address: Department of Mathematics, University of California, Los Angeles, California 90095 